\title{
Portfolio Efficiency with High-Dimensional Data as Conditioning Information
}

\author{
Caio Vigo Pereira ${ }^{\dagger}$ \\ ${ }^{\dagger}$ Department of Economics - University of Kansas
}

September 14, 2020

\begin{abstract}
In this paper, we build efficient portfolios using different frameworks proposed in the literature with several datasets containing an increasing number of predictors as conditioning information. We carry an extensive empirical study to investigate several approaches to impose sparsity and dimensionality reduction, as well as possible latent factors driving the returns of the risky assets. In contrast to previous studies that made use of naive OLS and low-dimension information sets, we find that (i) accounting for large conditioning information sets, and (ii) the use of variable selection, shrinkage methods and factors models, such as the principal component regression and the partial least squares provide better out-of-sample results as measured by Sharpe ratios.
\end{abstract}

JEL classification: G11, G17, C32, C38.

Keywords: Dimensionality reduction. Shrinkage. Efficient Portfolios. Principal Components Regression (PCR). Partial Least Squares (PLS). Three-Pass Regression Filter (3PRF). Ridge Regression, LASSO.

Corresponding author - 1460 Jayhawk Blvd - Snow Hall, The University of Kansas, USA. email caiovigo@gmail.com 


\section{Introduction}

The use of return predictability to form efficient portfolios is a cornerstone in the asset pricing literature. Theoretically, this has been addressed in the mean-variance efficiency framework where many ways of bringing the conditioning information during the optimization process was proposed. Over the last decades, the literature has recognized some variables that have predictive power and therefore could be used as conditioning information. However, the search for all these instruments produced an enormous amount of variables that supposedly has or had some predictability power. This applies to firm characteristics, whole market financial variables, and macroeconomic datasets as well.

In this paper, we combine the nascent high-dimensional literature in finance to the above portfolio theory problem. We incorporate conditioning information in the efficient portfolios framework proposed in the literature making use of the recently developed statistical techniques for high-dimensional predictive estimations. Specifically, we build efficient portfolios using high-dimensional information sets as conditioning information and conduct an extensive out-of-sample analysis comparing the high-dimensional statistical methods with a naive OLS approach of dealing with a large set of potential conditioning information.

Since the purpose of using conditioning information is to provide signals about the state of the economy or the condition of the risky assets, exploring a diverse, large set of possibilities for the predictive instruments, as well as flexible functional forms in which these signals are used in the optimization process can enhance the construction of efficient portfolios. Another motivation for working with high-dimensional information sets can be related to the Hansen-Richard critique (see Cochrane, 2009). Even though there might be unobservable conditioning information used by economic agents, when dealing with a large set of potential candidates, we could think that enlarging this set increases the chance of capturing more information from a wide variety of heterogeneous economic agents' information sets. On top of that, with the use of recently proposed measures of economic uncertainty (see Baker et al., 2016; Püttmann, 2018; Jurado et al., 2015), we could partially capture some of what used to be non-observable information.

We exploit the wealth set of data as predictive signals and condense them when estimating the conditional mean using high-dimensional data. We do this using many different techniques in order to impose sparsity and dimensionality reduction when finding the conditional mean, which is the most important driver in the formation of mean-variance efficient portfolios with conditioning information.

In short, we make a "bet in sparsity", in the sense that much of the information in the conditioning set can be summarized by few factors. We evaluate how penalized estimators, 
such as LASSO, Ridge and Elastic Net, as well as pure dimensionality reduction and latent factors approaches as Partial Least Squares (PLS) and Principal Components Regression (PCR), in addition to a generalization of the former (Three Pass Regression Filter) can produce different optimized portfolios.

This study makes a number of contributions to the literature. Assessing these methods in a high-dimensional setting for the collection of instruments, we show that it is possible to build efficient portfolios, for different efficient portfolios construction approaches, delivering on average higher out-of-sample Sharpe ratios, implied Sharpe ratios, and higher certainty equivalent returns (CER). We find that PLS and PCR can enhance the formation of optimized portfolios, generating large and significant alphas when evaluated in factor models, such as Fama-French 3 and 5. We also evaluate which one, among the large set of signals, have larger impacts in the excess returns conditional means.

From the theoretical implications derived by Hansen and Richard (1987) Hansen and Richard (1987), we could split in two main ways of defining the minimum variance efficient portfolios: the straightforward conditionally mean-variance (CMV), and the unconditionally mean-variance with conditioning information (UMV) portfolios. The first approach (CMV) is the most direct way to incorporate conditioning information. On the other hand, with roots on information asymmetry, UMV was studied in Ferson and Siegel (2001) who presented closed-form solutions. UMV is a more interesting and applicable way of working with conditioning information. For the sake of completeness, we also assess a modern portfolio management version of UMV proposed by Chiang (2015), known as mean-variance active tracking error (MVATE).

The dimension reduction literature is a growing topic having produced so far many methods to deal with high-dimensional datasets. One of the most straightforward and commonly used technique is based on the principal components analysis (PCA). The principal components regression (PCR) is a dimension reduction technique based on PCA. Several studies in macroeconomics made use of principal components to condense information (see Stock and Watson (2002a b) ). Kelly et al. (2019) proposed a dimension reduction based on PCA to explain the cross-section of average returns. Gu et al. (2018) made use of PCR, among other machine learning techniques, for measuring asset risk premia. Another classical dimension reduction technique is the partial least squares (PLS). PLS is a simple regression-based procedure designed to parsimoniously forecast a single time series using a large panel of predictors. Light et al. (2017) uses PLS to estimate the expected returns on individual stocks from a large set of firm characteristics.

A recent method was proposed by Kelly and Pruitt (2015). The Three-Pass Regression Filter (3PRF) is a generalization of PLS that can be represented as a set of ordinary regres- 
sion filter. The $3 \mathrm{PRF}$ is a constrained least squares estimator and reduces to partial least squares as a special case. Kelly and Pruitt (2013) uses the 3PRF to forecast the conditional expectation of market returns and cash flow.

A growing and nascent literature in finance has started to apply penalized regression in asset pricing. Kozak et al. (2019) adapt Ridge and LASSO estimators to estimate the stochastic discount factor to summarize and impose sparsity of an extensive list of predictors in a high-dimension setting. Freyberger et al. (2017) use a variation of LASSO to select characteristics from a large number of characteristics and estimate a nonlinear function for expected returns. Chinco et al. (2019) use LASSO to make intraday return forecasts for a large number of stocks. Other papers made use of penalized estimators for macro variables and bond risk premia as well (see Bianchi et al. (2019); Huang and Shi (2011); Bai and Ng $(2006))$. In section 3 we present these estimators.

The structure of this paper is as follows. Next section introduces the general framework and presents the three approaches mentioned above, the optimization problems and the weights' solution of building mean-variance efficient portfolios. Section 3 summarizes the techniques to impose sparsity in a high-dimensional setting, presenting the penalized estimators, the PLS, PCR, and the 3PRF. Section 4 explains our research design, explaining the datasets used, and how the performance is evaluated. Section 5 presents the results. Finally, section 6 concludes. Additional results, tables and figures are presented in the Internet Appendix.

\section{General Framework}

Consider an investment set with $N$ risky assets, indexed by $i=1, \ldots, N$ and the existence of a risk-free asset. Let $R_{f, t}$ be the return of the risk-free asset at time $t$, and denote $\mathbf{R}_{\rfloor^{1}}^{1}$ as the $N$-dimensional vector with the gross returns of the $N$ risky assets at time $t$. The vector of excess of returns $\mathbf{r}_{t}$ at time $t$ is given by $\mathbf{r}_{t}=\mathbf{R}_{t}-\mathbf{1} R_{f}$, where $\mathbf{1}$ is a an $N$-dimensional vector of ones. Let $\mathbf{x}_{t}\left(\mathbf{Z}_{t}\right)$ denote the vector of portfolio weights on the $N$ risky assets at time $t$, which is a function of the investor's conditioning information $\mathbf{Z}_{t}$ at time $t$. At each period of time $t$, using the conditioning information $\mathbf{Z}_{t}$ the investor set the weights of the vector $\mathbf{x}_{t}$, investing the remaining of the funds in the risk-free asset. Thus, the weight on the risk-free asset is given by $1-\mathbf{x}_{t}\left(\mathbf{Z}_{t}\right)^{\top} \mathbf{1}$. Therefore, the final payoff of the portfolio at $t+1$ is given by:

\footnotetext{
${ }^{1} \mathrm{~A}$ note on the notation throughout our analysis. Unless mentioned otherwise, we use lowercase boldface characters to represent vectors (except for $\mathbf{R}_{t}$ ), uppercase boldface characters for matrices, and regular characters for scalars.
} 


$$
\begin{aligned}
R_{p, t+1} & =\mathbf{R}_{t+1}^{\top} \mathbf{x}_{t}\left(\mathbf{Z}_{t}\right)+\left(1-\mathbf{x}_{t}\left(\mathbf{Z}_{t}\right)^{\top} \mathbf{1}\right) R_{f, t+1} \\
& =\left(\mathbf{R}_{t+1}-\mathbf{1} R_{f, t+1}\right)^{\top} \mathbf{x}_{t}\left(\mathbf{Z}_{t}\right)+R_{f, t+1} \\
& =\mathbf{x}_{t}\left(\mathbf{Z}_{t}\right)^{\top} \mathbf{r}_{t+1}+R_{f, t+1}
\end{aligned}
$$

In general, we assume that we can model the data generating process of the excess returns of each one the risky assets as

$$
r_{i, t}=\mu_{i, t}\left(\mathbf{Z}_{t-1}\right)+\epsilon_{i, t}
$$

where $\mu_{t}$ conditional expected excess returns of asset $i$ at time $t$, and $\epsilon_{i, t}$ is the noise term with conditional mean equal to 0 given $\mathbf{Z}_{t-1}$. Equation (4) highlights that this conditional mean is a function of a set of variables that enters this function lagged one period, seeking to span the set of available information. The fundamental idea is that this conditional mean is a function that can be broadly represented as

$$
\mu_{i, t}\left(\mathbf{Z}_{t-1}\right)=h_{i}\left(\mathbf{Z}_{t-1}\right)
$$

where $h_{i}(\cdot)$ is any function to approximate the conditional mean. Previous studies that analyzed empirically efficient portfolios for a specific market and asset class, predominantly made use of OLS to estimate $\boldsymbol{\mu}_{t}\left(\mathbf{Z}_{t-1}\right)$ when deriving the efficient portfolios weights from the mean-variance optimization problem, setting $\mu_{i, t}\left(\mathbf{Z}_{t-1}\right)=\mathbb{E}\left(r_{i, t} \mid \mathbf{Z}_{t-1}\right)$ from a standard linear regression. This was mainly motivated by the fact they used a small number of instruments as conditioning information set. Most of them have used at most a handful of variables. The choices of these variables were mostly motivated by previous studies that have found some predictability power for a specific variable in a specific period in the past. In this fashion, they argued that the conditional expected return for each asset $i=1, \ldots, N$, could be obtained by running the following regression:

$$
\mathbf{r}_{i}=\mathbf{Z}_{t-1} \boldsymbol{\theta}_{i}+\boldsymbol{\epsilon}_{i}
$$

where $\mathbf{Z}_{t-1}$ is a $T \times K$ matrix of lagged instruments and $\boldsymbol{\theta}_{i}$ is $K$-dimensional vector of parameters. Using the fitted values of the times series from equation (6) as the sample counterpart of $\boldsymbol{\mu}_{t}$, as follows 


$$
\widehat{\boldsymbol{\mu}}_{t}=\left[\begin{array}{c}
\widehat{\mu}_{1, t}\left(\mathbf{Z}_{t-1}\right) \\
\widehat{\mu}_{2, t}\left(\mathbf{Z}_{t-1}\right) \\
\vdots \\
\widehat{\mu}_{N, t}\left(\mathbf{Z}_{t-1}\right)
\end{array}\right]
$$

it is possible to find the solutions of the optimal portfolios weights.

However, in doing so, we face several issues. First, as Welch and Goyal (2007) shows in a comprehensive study, most of the variables taken as having predictability power and commonly used in academic research and by market practitioners perform badly out-ofsample, and in some cases even in the in-sample analysis. The authors selected variables from previously published articles and show that most of them behave poorly with unstable predictive performance.

Second, even in the case, a variable has some predictability power, this can only exist for a specific period (perhaps only in the past). Welch and Goyal (2007) sheds light on, among other things, to the problem of short-lived predictors, in contraposition to steady long-lived predictors. Some of the variables could have some predictive power that existed in a specific period. In a broad analysis McLean and Pontiff (2016) evaluated the outof-sample post-publication return predictability of 97 signals from 79 different academic studies. They show that after an academic paper is published, the average predictor's longshort return shrinks post-publication'? They also show that the publication has an impact on the predictor portfolio returns. They argue that post-published correlation with alreadypublished predictor portfolio returns increases.

Third, as Chinco et al. (2019) mentions, modern financial markets are big, fast, and complex. With access to so many sources, the process of data collection and processing reached the level of commonly known as big data. As Diebold et al. (2019) summarizes, in time-series regression involving $T$ time periods, $K$ regressors and $N$ dimensions (multivariate), we need to deal with some different characteristic data. As $K$ gets large (e.g. $K>T$ ) we have what is called as wide data, while as $N$ gets large we have high-dimensional data. It is known that in such cases the ordinary least squares (OLS) estimates will have a poor behavior in forecasting, or not even exist for wide data for instance. An appropriate way to deal with these types of data is via some sort of shrinkage-type regularization. Sparsity and dimensionality reduction are the goals of these problems. There are compelling arguments that support that we cannot rely on a small set of conditioning information. In order to obtain

\footnotetext{
${ }^{2}$ Portfolio returns dropped $26 \%$ out-of-sample and $58 \%$, yielding an estimated effect of $32 \%(58 \%-26 \%)$ lower return from publication-informed trading.
} 
better approximations to the conditional mean, the set of instruments must be enriched to resemble the exponential growth of available information.

The financial literature has collected a large list of variables that could be part of the conditioning information set, as pointed by Cochrane (2011). Several recent studies tried to compile these possible predictors. Green et al. (2013) assessed 330 signals (stock-level) reported in the past years. Feng et al. (2017) studied a list of 150 risk factors, what they called a "zoo" of factors proposed in the literature in the past 30 years. Harvey et al. (2016) analyzed the statistical significance of more than 300 signals published over the last forty years.

Finally, finding the conditional mean to explain the variation of the risky assets, is a risk premia problem. By far, it is known that the problem of explaining the risk premia has been one of the most debated and scrutinized topics for a long period in the literature. Clearly, approximating $h_{i}(\cdot)$ is a hard task because market efficiency forces return variation to be dominated by unforecastable news that obscures risk premia Gu et al. (2018). We can say that not only the amount, but also the complexity of information driving the conditional mean of the risky assets could be enhanced. Modeling the conditional mean with few observable factors entering linearly, with no interactions, penalization, or dimension reduction techniques, could also be an incomplete structure to explain the stochastic discount factor.

Hence, in order to exploit the wealth of data and try to condense them when estimating the conditional mean using high-dimensional data, we seek to impose sparsity and dimensionality reduction when finding the conditional mean that enters in all functions to build efficient portfolios. Importantly, it is the fact that we can consider the function $h_{i}(\cdot)$ in equation 5 that seeks to approximate the conditional mean driving the DGP of the excess

returns to have a rich and flexible format. In order to obtain better approximations, we assume that $h_{i}(\cdot)$ is asset dependent, in the sense that the factors that drive the excess returns of each risky asset $i$ are built using a high-dimensional data for each different asset.

\subsection{Optimal Portfolios with Conditioning Information}

In this section, we briefly present some of the approaches that were proposed in the literature to build efficient portfolios with conditioning information, summarizing three frameworks.

\subsubsection{Conditionally Efficient Portfolios}

As in Hansen and Richard (1987), the conditionally efficient returns is the solution of the following problem: 


$$
\begin{array}{cc}
\min _{x(Z)} & \mathbb{E}\left(r_{p, t+1}^{2} \mid \mathbf{Z}_{t}\right) \\
\text { s.t. } & \mathbb{E}\left(r_{p, t+1} \mid \mathbf{Z}_{t}\right)=\alpha_{p, t+1}
\end{array}
$$

where $r_{p, t+1}$ is the portfolio excess returns at time $t+1$, and $\alpha_{p, t+1}$ is the target conditional expected excess return. The solution of the problem in equation (8) providing the weights allocated in each risky asset is given by:

$$
\mathbf{x}_{t}\left(\mathbf{Z}_{t-1}\right)=\left(\frac{\mathbb{E}\left(r_{p, t+1} \mid \mathbf{Z}_{t}\right)}{\boldsymbol{\mu}_{t}\left(\mathbf{Z}_{t-1}\right)^{\top}\left(\boldsymbol{\Gamma}_{t}\left(\mathbf{Z}_{t-1}\right)\right)^{-1} \boldsymbol{\mu}_{t}\left(\mathbf{Z}_{t-1}\right)}\right)\left(\boldsymbol{\Gamma}_{t}\left(\mathbf{Z}_{t-1}\right)\right)^{-1} \boldsymbol{\mu}_{t}\left(\mathbf{Z}_{t-1}\right)
$$

where $\boldsymbol{\mu}_{t}\left(\mathbf{Z}_{t-1}\right)$ is the $n$-dimensional vector of conditional expected excess returns at time $t$, and $\boldsymbol{\Gamma}_{t}\left(\mathbf{Z}_{t-1}\right)$ is the $N \times N$ conditional second moment matrix at time $t$.

\subsubsection{Unconditionally Efficient Portfolios with Respect to the Information}

The unconditionally mean-variance (UMV) efficient portfolio strategy takes another path. Ferson and Siegel (2001) study the properties of unconditional minimum-variance portfolios in the presence of conditioning information. As the authors argue, whenever the investor makes use of conditioning information, mean-variance efficiency can be either defined in terms of the two first conditional moments (as in section 2.1.1), or the unconditional moments as in the UMV approach. Here, the objective is to maximize the unconditional mean relative to the unconditional variance, being the strategies a function of the available information.

Hansen and Richard (1987) show that unconditionally efficient returns are a subset of conditionally efficient returns. Related with information asymmetry, this is a typical situation where a portfolio manager using conditioning information builds a conditionally efficient portfolio. However, for an uninformed investor, the portfolio does not appear efficient. As in Ferson and Siegel (2001), the solution of the UMV problem providing the weights allocated in each risky asset is given by:

$$
\mathbf{x}_{t}\left(\mathbf{Z}_{t-1}\right)=\left(\frac{\mathbb{E}\left(r_{p, t+1}\right)}{\mathbb{E}\left(\boldsymbol{\mu}_{t}\left(\mathbf{Z}_{t-1}\right)^{\top}\left(\boldsymbol{\Gamma}_{t}\left(\mathbf{Z}_{t-1}\right)\right)^{-1} \boldsymbol{\mu}_{t}\left(\mathbf{Z}_{t-1}\right)\right)}\right)\left(\boldsymbol{\Gamma}_{t}\left(\mathbf{Z}_{t-1}\right)\right)^{-1} \boldsymbol{\mu}_{t}\left(\mathbf{Z}_{t-1}\right)
$$

where $\mathbb{E}\left(r_{p, t+1}\right)$ is the unconditional expected return target, and $\mathbb{E}\left(\boldsymbol{\mu}_{t}\left(\mathbf{Z}_{t-1}\right)^{\top}\left(\boldsymbol{\Gamma}_{t}\left(\mathbf{Z}_{t-1}\right)\right)^{-1} \boldsymbol{\mu}_{t}\left(\mathbf{Z}_{t-1}\right)\right)$ is the unconditional mean of $\boldsymbol{\mu}_{t}\left(\mathbf{Z}_{t-1}\right)^{\top}\left(\boldsymbol{\Gamma}_{t}\left(\mathbf{Z}_{t-1}\right)\right)^{-1} \boldsymbol{\mu}_{t}\left(\mathbf{Z}_{t-1}\right)$. 


\subsection{Unconditionally Tracking Efficient Portfolios}

Chiang (2015) follows a different approach to exploit conditioning information in the mean-variance framework. The author uses tracking error strategies, in which an active portfolio manager uses conditioning information to optimize unconditional performance measures relative to a benchmark. He calls this approach as unconditional tracking efficiency. The author mentions that this structure should be seen as a common practice between market practitioners, since a portfolio manager conducting an optimization uses more information than his clients, that possibly do not have access to the entire set being used as conditioning information, driving managers to seek higher performance from the clients' perspective.

In this framework, consider a portfolio manager who uses conditioning information $\mathbf{Z}_{t}$, which is not available to his clients, to build a vector $\mathbf{x}_{t}\left(\mathbf{Z}_{t-1}\right)$ of unrestricted weights of the $N$ available risky assets. Under the unconditional mean-variance tracking error approach (UMVTE), this active manager uses conditioning instruments to form portfolios that optimize unconditional performance measure. Thus, for a benchmark $b$, with returns given by $R_{b, t}$, the UMVTE problem is to minimize the unconditional error variance, $\operatorname{Var}\left(R_{p, t+1}-R_{b, t+1}\right)$, for a given unconditional expected return target $\alpha_{p, t+1}=\mathbb{E}\left(R_{p, t+1}-\right.$ $\left.R_{b, t+1}\right)$,

$$
\begin{array}{cc}
\min _{\mathbf{x}(\mathbf{Z})} & \mathbb{V a r}\left(R_{p, t+1}-R_{b, t+1}\right) \\
\text { s.t. } & \mathbb{E}\left(R_{p, t+1}-R_{b, t+1}\right)=\alpha_{p, t+1}
\end{array}
$$

Chiang (2015) solves this minimization problem using calculus of variations. The unique solution to the problem given on equation 11 without constraint on portfolio risk is determined by the following function for the portfolios weights:

$$
\mathbf{x}_{t}\left(\mathbf{Z}_{t-1}\right)=\lambda_{1}\left(\boldsymbol{\Gamma}_{t}\left(\mathbf{Z}_{t-1}\right)\right)^{-1} \boldsymbol{\mu}_{t}\left(\mathbf{Z}_{t-1}\right)+\left(\boldsymbol{\Gamma}_{t}\left(\mathbf{Z}_{t-1}\right)\right)^{-1} \boldsymbol{\gamma}_{t}\left(\mathbf{Z}_{t-1}\right)
$$

where, $\boldsymbol{\mu}_{t}\left(\mathbf{Z}_{t-1}\right)$ is the conditional mean, $\boldsymbol{\Gamma}_{t}\left(\mathbf{Z}_{t-1}\right)$ is the conditional second moment of the excess returns, $\boldsymbol{\gamma}_{t}\left(\mathbf{Z}_{t-1}\right)=\mathbb{E}\left(\mathbf{r}_{t}\left(r_{b, t}-\mu_{b, t}\right) \mid \mathbf{Z}_{t-1}\right)$, and $\lambda_{1}$ an scalar $3^{3}$. For a full proof of the solution of the UMVTE problem, see Chiang (2015). Note that the CMV, UMV and UMVTE are not the only frameworks proposed in the literature to build efficient portfolios taking into account conditioning information. Brandt and Santa-Clara (2006) Peñaranda (2016) also proposed different approaches to build mean-variance efficient portfolios with

\footnotetext{
${ }^{3}$ Precisely,

$$
\lambda_{1}=\frac{\left(\alpha_{p, t+1}+\mu_{b, t}\right)-\eta_{2}}{\eta_{1}}
$$

where $\eta_{1}=\mathbb{E}\left(\boldsymbol{\mu}_{t}^{\top}\left(\mathbf{Z}_{t-1}\right)\left(\boldsymbol{\Gamma}_{t}\left(\mathbf{Z}_{t-1}\right)\right)^{-1} \boldsymbol{\mu}_{t}\left(\mathbf{Z}_{t-1}\right)\right)$, and $\eta_{2}=\mathbb{E}\left(\boldsymbol{\mu}_{t}^{\top}\left(\boldsymbol{\Gamma}_{t}\left(\mathbf{Z}_{t-1}\right)\right)^{-1} \boldsymbol{\gamma}_{t}\left(\mathbf{Z}_{t-1}\right)\right)$
} 
conditioning information.

\section{Estimation}

In this section we summarize some of the approaches proposed in the current state of the literature to deal with high-dimensional set of predictors, imposing sparsity through shrinkage and selection type techniques, and latent variables estimators as well.

\subsection{Penalized Regression}

\subsubsection{LASSO}

Consider the regression in equation (6). In general, the OLS regression to obtain the $K$ parameters of $\boldsymbol{\theta}_{i}$ will provide nonzero estimates. When dealing with high-dimensional with a large number of predictors, usually we face two issues using OLS. First, whenever we have $K>T$, OLS cannot find a unique solution. Second, the infinite set of solutions have a tendency to overfit the data instead of extracting informative signals for the conditional mean. This fact, known as "curse of dimensionality" is well known in the literature. Gu et al. (2018) mention that this issue is more exasperating where the signal-noise ratio in return predictions is low. This causes the OLS estimations to have a poor behavior in forecasting.

To deal with the inconsistency and the inefficiency of the OLS in high-dimensional data, shrinking the number of estimated parameters is essential to avoid overfitting the data. Thus, some sort of shrinkage or variable selection might be necessary to deal with this type of data. This can be done penalizing the objective function being optimized. In general, penalized estimators share the following common structure:

$$
\mathcal{L}(\boldsymbol{\theta} ; \cdot)=\underbrace{\mathcal{L}(\boldsymbol{\theta})}_{\text {Loss Function }}+\underbrace{\phi(\boldsymbol{\theta} ; \cdot)}_{\text {Penalty }}
$$

An estimator to impose some sort of sparsity in the set of regressors is the Least Absolute Selection and Shrinkage Operator (LASSO), proposed in the seminal paper by Tibshirani (1996). This estimator regularizes the estimation process constraining the size of the estimates. Following Hastie et al. (2015); Friedman et al. (2001), the LASSO is a $\ell_{1}$ regularized regression obtained by minimizing the following problem: 


$$
\begin{gathered}
\hat{\boldsymbol{\theta}}_{i}^{L A S S O}=\arg \min _{\theta_{0, i}, \boldsymbol{\theta}_{i}}\left\{\frac{1}{T} \sum_{t=1}^{T}\left(r_{i, t}-\theta_{0, i}-\sum_{k=1}^{K} Z_{k, t-1} \theta_{k, i}\right)^{2}\right\} \\
\text { s.t. }\|\theta\|_{1} \leq c
\end{gathered}
$$

where $\|\theta\|_{1}=\sum_{k=1}^{K}\left|\theta_{k, i}\right|$ is the $\ell_{1}$ norm of $\theta$. We call $c$ the "parameter budget", being possible to interpret $\boldsymbol{\theta}_{i}^{L A S S O}$ as the best fit within this budget ${ }^{4}$. The parameter $\lambda$ controls the amount the shrinkage, the larger it is, the greater is the shrinkage. The penalty imposed in equation (17) makes the solutions nonlinear in $r_{i, t}$. This is associate with the fact that there is no closed-form solution. The lasso problem is a convex program, and computing its solution is a quadratic programming problem. An important feature of LASSO is that making $c$ sufficiently small, this estimator sets a subset of the estimated coefficients to exactly zero in order to impose sparsity.

\subsubsection{Ridge}

The ridge estimator is similar to the LASSO. However, ridge sets the penalty $\phi(\boldsymbol{\theta} ; \cdot)$ as $\|\theta\|_{2}^{2}=\sum_{k=1}^{K} \theta_{k, i}^{2}$, i.e., as the penalty is a $\ell_{2}$ norm. Thus, we we can it write as

$$
\hat{\boldsymbol{\theta}}_{i}^{\text {Ridge }}:=\left\{\arg \min _{\theta_{0, i}, \boldsymbol{\theta}_{i}} \frac{1}{T} \sum_{t=1}^{T}\left(r_{i, t}-\theta_{0, i}-\sum_{k=1}^{K} Z_{k, t-1} \theta_{k, i}\right)^{2}+\lambda \sum_{k=1}^{K} \theta_{k, i}^{2}\right\} .
$$

An important characteristic of Ridge approach is the one-to-one correspondence between the parameters $\lambda$ and $t$. This fact makes Ridge estimation to attenuate multicollinearity, when it is present in the data. Since, when $K$ is large, the large number of regressors may result in high correlation among some of them. With standard "naive" OLS, multicollinearity causes poorly determined coefficients with large variances (and large variance inflator factor - VIF), what Ridge can mitigate.

${ }^{4}$ Equivalently, in Lagrange-multiplier form we can it write as,

$$
\hat{\boldsymbol{\theta}}_{i}^{L A S S O}:=\arg \min _{\theta_{0, i}, \boldsymbol{\theta}_{i}}\left\{\frac{1}{T} \sum_{t=1}^{T}\left(r_{i, t}-\theta_{0, i}-\sum_{k=1}^{K} Z_{k, t-1} \theta_{k, i}\right)^{2}+\lambda \sum_{k=1}^{K}\left|\theta_{k, i}\right|\right\} .
$$




\subsubsection{Elastic Net}

Among many variants of LASSO, there is the Elastic Net (ENet) (Zou and Hastie, 2005). In summary, the ENet seeks to combine the penalties of both estimators above (LASSO and Ridge) via a convex combination. Therefore, the ENet estimator is given by,

$$
\hat{\boldsymbol{\theta}}_{i}^{E N e t}:=\left\{\arg \min _{\theta_{0, i}, \boldsymbol{\theta}_{i}} \frac{1}{T} \sum_{t=1}^{T}\left(r_{i, t}-\theta_{0, i}-\sum_{k=1}^{K} Z_{k, t-1} \theta_{k, i}\right)^{2}+\lambda \sum_{k=1}^{K}\left(\alpha\left|\theta_{k, i}\right|+(1-\alpha) \theta_{k, i}^{2}\right)\right\}(19
$$

where $\alpha \in[0,1]$. Setting $\alpha=1$ this estimator reduces to the LASSO regression with a $\ell_{1}$-norm, with $\alpha=0$ it reduces to the ridge regression with a $\ell_{2}$-penalty. Among the positive features of the ENet, when adding both penalties, this estimator automatically controls for strong within-group correlations. The ENet is also a strictly convex problem, thus, providing a unique solution independently of correlations or duplications in the $Z_{k, t-1}$. However, in equation 19 we have an additional tuning parameter $\alpha$ that has to be determined or defined ad hoc.

\subsection{Principal Components Regression (PCR)}

Whenever we are dealing with a high dimensional set of explanatory variables, high collinearities may be an issue to generate good conditional means estimates. One technique to deal with multicollinearity is the Principal Components Regression (PCR). PCR is a dimension reduction approach based on the Principal Components Analysis (PCA). Being a regression technique, PCR regresses the dependent variable on the principal components generated by the PCA.

In summary, PCR first performs PCA on the matrix of predictors (here, the $\mathbf{Z}_{t-1}$ of lagged instruments) and obtains the principal components associate with it. Out of the $M$ components, we can select a subset $m \in M$ and regress the dependent variable using the first $m$ principal components as regressor: 5 . Since PCR is essentially a shrinkage estimator that seeks to capture components with high varianc $\AA^{6}$, at the cost of those with low variance, this approach performs dimension reduction by selecting $m$ components, and also has a shrinkage effect as it removes those components with the low variance to form the final model to obtain the conditional mean.

Precisely, consider equation (6). Using the singular value decomposition (SVD), we

\footnotetext{
${ }^{5} \mathrm{~A}$ final step is done to scale back to the original regressors using the PCA loadings.

${ }^{6}$ Those with higher eigenvalues in $\mathbf{Z}^{\top} \mathbf{Z}$.
} 
can factorize $\mathbf{Z}^{7}$ as $\mathbf{Z}=\mathbf{U D V}^{\top}$, where $\mathbf{U}_{T \times K}$ and $\mathbf{V}_{K \times K}$ are orthogonal matrices 8 , and $\mathbf{D}_{K \times K}=\operatorname{diag}\left[\delta_{1}, \delta_{2}, \ldots, \delta_{K}\right]$, being each $\delta_{m}$ the singular values of $\mathbf{Z}$.

We can obtain the principal values of $\mathbf{Z}^{\top} \mathbf{Z}$ as $\boldsymbol{\Lambda}_{K \times K}=\operatorname{diag}\left[\delta_{1}^{2}, \delta_{2}^{2}, \ldots, \delta_{K}^{2}\right]$ through a spectral decomposition given by $\mathbf{V} \boldsymbol{\Lambda} \mathbf{V}^{\top}$. Finally, we can multiply the matrix $\mathbf{Z}$ by the first $m$ principal components $\mathbf{V}^{(m)}=\left[\boldsymbol{\omega}^{(1)}, \ldots, \boldsymbol{\omega}^{(m)}\right]$, where $m \in\{1,2, \ldots, K\}$, to obtain our derived covariates. Each $m$ principal component $\boldsymbol{\omega}^{(m)}$ can be seen as $m$ linear combinations of $\mathbf{Z}_{t-1}$. Thus, using the PCR approach, the conditional mean at time $t$ of the excess returns of risky asset $i$ can be approximated running the following regression

$$
\mathbf{r}_{i}=\underbrace{\left(\mathbf{Z}_{t-1} \mathbf{V}_{t-1}^{(m)}\right)}_{\text {Derived Covariates }} \boldsymbol{\theta}_{i}^{(m)}+\boldsymbol{\epsilon}_{i}
$$

so that $\widehat{\boldsymbol{\mu}}_{i}\left(\mathbf{Z}_{t-1}\right)=\left(\mathbf{Z}_{t-1} \mathbf{V}_{t-1}^{(m)}\right) \hat{\boldsymbol{\theta}}_{i}^{(m)}$, where $\hat{\boldsymbol{\theta}}_{i}^{(m)}=\left(\mathbf{V}_{t-1}^{(m) \top} \mathbf{Z}_{t-1}^{\top} \mathbf{Z}_{t-1} \mathbf{V}_{t-1}^{(m)}\right) \mathbf{V}_{t-1}^{(m) \top} \mathbf{Z}_{t-1}^{\top} r_{i, t}$. Equation 20 makes clear that PCR reduces dimensions by using $m$ principal components that capture the largest common variation in the conditioning information set, and weights through $\boldsymbol{\omega}^{(m)}$ the original covariates. The $m$ PCA components used to form the derived covariates in the final model is a parameter that needs to be chosen adaptively. In section 4.1 we discuss how this is done.

\subsection{Partial Least Squares (PLS)}

When PCR finds the components with the largest common variation in the conditioning information set, it takes into consideration in its objective function only the information from $\mathbf{Z}_{t-1}$. As $\mathrm{Gu}$ et al. (2018) argues, doing so it fails to incorporate the information from the dependent variable (excess returns) when performing dimension reduction, which can be suboptimal to generate good conditional means approximations. The Partial Least Squares (PLS), on the other hand, uses the common components from $\mathbf{Z}_{t-1}$ by conditioning on the joint distribution of $r_{i, t}$ and $\mathbf{Z}_{t-1}$. For this reason, PLS is considered a latent approach that models the covariance between the spaces generated by both matrices $\left(r_{i, t}\right.$ and $\left.\mathbf{Z}_{t-1}\right)$.

As mentioned by Friedman et al. (2001) this method seeks to obtain the directions that provide the highest variance and highest correlation $!^{9}$ between $r_{i, t}$ and $\mathbf{Z}_{t-1}$ Stone and Brooks (1990); Frank and Friedman (1993). Following Friedman et al. (2001), the idea of PLS is

\footnotetext{
${ }^{7}$ We dropped the time subscripts $t-1$ for simplicity.

${ }^{8}$ These matrices are known as left and right singular vectors of $\mathbf{Z}$ respectively.

${ }^{9} \mathrm{~A}$ clear contrast to PCR that only seeks directions that maximize only the variance between $r_{i, t}$ and $\mathbf{Z}_{t-1}$
} 
to weight each vector $\mathbf{z}_{t-1, k} \in \mathbf{Z}_{t-1}$ by its partial least squares direction $\hat{\varphi}_{k}=\left\langle\mathbf{z}_{t-1, k}, r_{i, t}\right\rangle$. The derived input $\mathfrak{z}_{k}=\sum_{k} \widehat{\varphi}_{k} \mathbf{z}_{t-1, k}$ is then used to obtain the the estimated coefficient $\widehat{\theta}_{k}$ regressing $r_{i, t}$ on the derived input $\mathfrak{z}_{k}$. This is done from $k=1,2, \ldots, K$, where we orthogonalize the original predictors with respect to the previous component. This process repeats until $m \leq K$ desired directions to reduce dimensions have been found. Clearly, if we build $m=K$ directions, we return to the standard least squares estimates.

The idea of the partial least squares direction $\hat{\varphi}_{k}$ is to obtain the weight of the strength (or the partial sensitivity) of the univariate effect each variable in the conditioning set $\mathbf{Z}_{t-1}$ on $r_{i, t}$. As with the PCR, the $m$ number of directions is a parameter that needs to be chosen appropriately.

\subsection{Three-Pass Regression Filter (3PRF)}

Kelly and Pruitt (2015) proposed the three-pass regression filter (3PRF), a generalization of the PLS estimator, in the sense that the latter is a special case of the former. In this setting, assuming that the data can be represented by an approximate factor model to reduce the dimension of the predictive information, the target variable are the time series of the excess returns of each risky asset $i$, having the following general form: $r_{t+h}^{i}=\beta_{0}^{i}+\boldsymbol{\beta}^{i^{\top}} \boldsymbol{F}_{t}^{i}+\eta_{t+h}^{i}$. Denoting by $\boldsymbol{r}^{i}$ the $T \times 1$ vector of the excess returns for asset $i$, we can write in matrix notation the target variable as:

$$
\begin{aligned}
\boldsymbol{r}^{i} & =\left[\begin{array}{c}
r_{t+1}^{i} \\
r_{t+2}^{i} \\
\vdots \\
r_{t+T}^{i}
\end{array}\right] \\
\boldsymbol{r}^{i} & =\mathbf{1} \beta_{0}^{i}+\boldsymbol{F}^{i} \boldsymbol{\beta}^{i}+\boldsymbol{\eta}^{i} \quad i=1,2, \ldots, N
\end{aligned}
$$

where $\mathbf{1}$ is again a $T$-dimensional vector of ones, $\beta_{0}^{i}$ is a scalar, $\boldsymbol{F}^{i}$ is a $T \times K_{F}$ matrix of factors, $\boldsymbol{\beta}^{i}$ is a $K_{F}$-dimensional vector of parameters, and $\boldsymbol{\eta}^{i}$ is a $T$-dimensional vector of errors, all defined for the risky asset $i$.

Denoting by $\mathbf{Z}$ the $T \times L$ matrix of conditioning information used as predictors, the cross-section of all the $L$ variables forming the conditioning information is represented by $z_{l, t}=\phi_{l, 0}+\boldsymbol{\phi}_{l}^{\top} \boldsymbol{F}_{t}+\epsilon_{l, t}$, for $l=1,2, \ldots, L$. Defining $\boldsymbol{z}_{t}=\left(z_{1, t}, z_{2, t}, \ldots, z_{L, t}\right)^{\top}$, then the matrix representation of $\boldsymbol{Z}$ is given by 


$$
\begin{aligned}
\boldsymbol{Z} & =\left[\begin{array}{c}
\boldsymbol{z}_{1} \\
\boldsymbol{z}_{2} \\
\vdots \\
\boldsymbol{z}_{T}
\end{array}\right]=\left[\begin{array}{cccc}
z_{1,1} & z_{2,1} & \ldots & z_{3,1} \\
z_{1,2} & z_{2,2} & \ldots & z_{3,2} \\
\vdots & \vdots & \ldots & \vdots \\
z_{1, T} & z_{2, T} & \ldots & z_{3, T}
\end{array}\right] \\
& =\mathbf{1} \boldsymbol{\phi}_{0}^{\top}+\boldsymbol{F} \boldsymbol{\Phi}^{\top}+\boldsymbol{\varepsilon}
\end{aligned}
$$

where $\mathbf{1}$ is a $T$-dimensional vector of ones, $\phi_{0}$ is a $L \times 1$ vector of intercepts, $\boldsymbol{F}$ is the $T \times K_{F}$ matrix of latent factors, $\boldsymbol{\Phi}=\left[\boldsymbol{\phi}_{1}, \boldsymbol{\phi}_{2}, \ldots, \boldsymbol{\phi}_{L}\right]^{\top}$ with dimension $L \times K_{F}$, and $\varepsilon$ is a $T$-dimensional vector of errors.

For each risky asset $i$ in $i=1,2, \ldots, N$ there are three steps. The first pass of the estimator runs $L$ separate time series regressions (for a fixed risky asset $i$ ), i.e., one for each variable forming our conditioning information on the forecast target:

$$
z_{l, t}^{i}=\hat{\phi}_{l, 0}^{i}+\hat{\phi}_{l}^{i} r_{t+h}^{i}+e_{l, t}^{i}
$$

The estimated coefficients $\hat{\phi}_{l}$ describe the sensitivity of conditioning information (predictors) to the latent factor driving the forecast target.

In the second pass, we use the estimated first-pass coefficients in $T$ separate cross section regressions, where our conditioning information variable $l$ are the dependent variable, and the first-stage loadings $\hat{\phi}_{l}^{i}$ are the independent variables

$$
z_{l, t}^{i}=\hat{c}_{t}^{i}+\boldsymbol{F}_{t}^{i} \hat{\phi}_{l}^{i}+u_{l, t}^{i}
$$

The first-stage coefficient estimates map the cross-sectional distribution of predictors to the latent factors. Second-stage cross section regressions use this map to back out estimates of the factors at each point in time ((Kelly and Pruitt, 2015) $)$.

Table 1: The Three Pass Regression Filter (3PRF)

\begin{tabular}{cl}
\hline Pass & Description \\
\hline 1 & run time series regression of $\boldsymbol{z}_{l}^{i}$ on $\mathbf{r}^{i}$ for $j=1,2, \ldots, L$ predictors \\
2 & run cross section regression of $\boldsymbol{z}_{l}^{i}$ on $\hat{\boldsymbol{\phi}}_{l}^{i}$ for $t=1,2, \ldots, T$ \\
3 & run time series regression of $r_{t+1}^{i}$ on predictive factors $\hat{\mathbf{F}}_{t}^{i}$ \\
\hline
\end{tabular}


The third stage is the final forecasting step. Running a single time series regression of the target variable $r_{t+1}^{i}$ on the second-pass estimated factors $\hat{\boldsymbol{F}}_{t}^{i}$, it provides the forecast, which

is the fitted value $r_{t+1}^{i}=\hat{\beta}_{0}^{i}+\hat{\boldsymbol{F}}_{t}^{i^{\top}} \hat{\boldsymbol{\beta}}^{i}$. The algorithm is summarized in table 1 . Kelly and Pruitt (2015) prove that, under a set of assumption ${ }^{10}$ the $3 \mathrm{PRF}$ is a consistent estimator as $L$ and $T$ become large. The authors also show that the 3PRF has a one-step closed form:

$$
\hat{\boldsymbol{r}}^{i}=\mathbf{1}_{T} \overline{\boldsymbol{r}}^{i}+\boldsymbol{J}_{T} \boldsymbol{Z} \boldsymbol{J}_{L} \boldsymbol{Z}^{\top} \boldsymbol{J}_{T} \boldsymbol{r}^{i}\left(\boldsymbol{r}^{i^{\top}} \boldsymbol{J}_{T} \boldsymbol{Z} \boldsymbol{J}_{L} \boldsymbol{Z}^{\top} \boldsymbol{J}_{T} \boldsymbol{Z} \boldsymbol{J}_{L} \boldsymbol{Z}^{\top} \boldsymbol{J}_{T} \boldsymbol{r}\right)^{-1} \boldsymbol{r}^{i^{\top}} \boldsymbol{J}_{T} \boldsymbol{Z} \boldsymbol{J}_{L} \boldsymbol{Z}^{\top} \boldsymbol{J}_{T} \boldsymbol{r}^{i}
$$

where $\overline{\boldsymbol{r}}^{i}=\mathbf{1}_{T}^{\top} \boldsymbol{r}^{i} / T$, for $\mathbf{1}_{T}$ a $T$-dimensional vector of ones, and $\boldsymbol{J}_{T} \equiv \boldsymbol{I}_{T}-\frac{1}{T} \mathbf{1}_{T} \mathbf{1}_{T}^{\top}$, for $\boldsymbol{I}_{T}$ a $T$-dimensional identity matrix, being $\boldsymbol{J}_{L}$ analogous.

\section{Empirical Strategy}

Our focus is from July 1963 to December 2017 for U.S. data. The out-of-sample cut off starts in Jan-1996 and we use recursive windows to update the estimations with the new available information. Using different estimators to impose dimensionality reduction, we compare the performance of each approach to build CMV, UMV and MVATE efficient portfolios, as presented on section 2.1.

At each $t$ we form the weights of our portfolios using equation (9) for the CMV, equation (10) for the UMV, and equation (12) for the UMVTE portfolio. For each one of them, we use the estimators presented in section 3 to obtain the conditional expected excess returns $\boldsymbol{\mu}_{t}$. To exploit the wealth of information available for predictors, we make use of high dimensional datasets as our conditioning information. Hence, we assess how PCR, PLS, 3PRF, LASSO, RIDGE, and the ENet behave, comparing them with our benchmark estimator, the OLS.

Thus, for all three methodologies we replace $\boldsymbol{\mu}_{t}\left(\mathbf{Z}_{t-1}\right)$ by its sample counterpart $\widehat{\boldsymbol{\mu}}_{t}\left(\mathbf{Z}_{t-1}\right)$ on $\mathbf{x}_{t}\left(\mathbf{Z}_{t}\right)$, being $\widehat{\boldsymbol{\mu}}_{t}\left(\mathbf{Z}_{t-1}\right)$ the fitted values of the estimation of the excess returns of all $N$ risky assets in the investment set on the $L$ lagged instruments. Here we have an important difference from previous studies. Ferson and Siegel (2009), Fletcher and Basu (2016), Abhyankar et al. (2012), Chiang (2015) among others have used standard OLS to obtain the fitted values, and a small set of instruments selected in advance as conditioning information.

Given that returns of risky assets are assumed to be generated by the conditional mean plus a noise term as in equation (4), we can estimate the conditional second moment matrix as which is given by:

\footnotetext{
${ }^{10}$ For further details, please refer to the original paper.
} 


$$
\begin{aligned}
\boldsymbol{\Gamma}_{t}\left(\mathbf{Z}_{t-1}\right) & =\mathbb{E}\left(\mathbf{r}_{t} \mathbf{r}_{t}^{\top} \mid \mathbf{Z}_{t-1}\right) \\
& =\boldsymbol{\Sigma}_{t}\left(\mathbf{Z}_{t-1}\right)+\boldsymbol{\mu}_{t}\left(\mathbf{Z}_{t-1}\right) \boldsymbol{\mu}_{t}\left(\mathbf{Z}_{t-1}\right)^{\top}
\end{aligned}
$$

where $\mathbf{r}_{t}$ is an $N \times 1$ vector of the $N$ risky assets, and $\boldsymbol{\Sigma}_{t}\left(\mathbf{Z}_{t-1}\right)$ is the nonsigular conditional covariance matrix of the noise. Plugging the estimates of the conditional expected excess returns on $\boldsymbol{\Gamma}_{t}\left(\mathbf{Z}_{t-1}\right)$, we can estimate the second moment matrix, assuming that the conditional covariance matrix $\boldsymbol{\Sigma}_{t}\left(\mathbf{Z}_{t-1}\right)$ is constant ${ }^{11}$. Notice, that $\boldsymbol{\Gamma}_{t}\left(\mathbf{Z}_{t-1}\right)$ is time-varying due to the time-varying characteristic of $\boldsymbol{\mu}_{t}\left(\mathbf{Z}_{t-1}\right)$. We estimate the conditional covariance matrix $\Sigma_{t}\left(\mathbf{Z}_{t-1}\right)$ as the estimate of the residual covariance matrix obtained with the regressions for $\hat{\boldsymbol{\mu}}_{t}\left(\mathbf{Z}_{t-1}\right)$.

Following Fletcher and Basu (2016), we also calculate $\mathbb{E}\left(\boldsymbol{\mu}_{t}\left(\mathbf{Z}_{t-1}\right)^{\top}\left(\boldsymbol{\Gamma}_{t}\left(\mathbf{Z}_{t-1}\right)\right)^{-1} \boldsymbol{\mu}_{t}\left(\mathbf{Z}_{t-1}\right)\right)$ on equation 100 as the average value of $\boldsymbol{\mu}_{t}\left(\mathbf{Z}_{t-1}\right)^{\top}\left(\boldsymbol{\Gamma}_{t}\left(\mathbf{Z}_{t-1}\right)\right)^{-1} \boldsymbol{\mu}_{t}\left(\mathbf{Z}_{t-1}\right)$ during the estimation window. Following Kirby and Ostdiek (2012) and Fletcher and Basu (2016) we set a small monthly target return. Thus, in equation (9) for the CMV, we set the conditional expected excess returns $\mathbb{E}\left(r_{p, t+1} \mid \mathbf{Z}_{t}\right)$ equal to $0.5 \%$. Similarly, in equation 10 for the UMV framework, we also set the unconditional expected excess returns $\mathbb{E}\left(r_{p, t+1}\right)$ equal to $0.5 \%$. For the UMVTE, we set $\alpha_{p}$ to be $\mathbb{E}\left(R_{p, t+1}-R_{b, t+1}\right)=0.5 \%$, where we consider the tracking portfolio to be the risk free asset of the economy.

\subsection{Validation}

All the techniques described in section 3 have hyperparameters that need to be chosen to define the final model. This process of determining the best hyperparameters is commonly known as tuning. As previously mentioned, penalized regressions do not have a closed form solution mainly because of the penalty $\lambda$ imposed in the objective function. For ENet, $\lambda$ and $\alpha$ need to be chosen, while for LASSO and RIDGE, only $\lambda$. For PCR, the number of principal components $m$ in $\mathbf{V}^{(m)}=\left[\boldsymbol{\omega}^{(1)}, \ldots, \boldsymbol{\omega}^{(m)}\right]$ need to be defined to determine the final regression model. For PLS, the $m$ number of principal directions should also be chosen 12 .

To tune the parameters above, we recursively define the training and testing periods. The prevailing approach in this literature is to use the tunning subsample to adaptively determine the hyperparameters. At each $\tau \in \tau_{O O S}$, where $\tau_{O O S}$ is the OOS subsample, we use all the previous information up to $\tau-1$ to build the training group. Within this group, the data ranges from $t=1,2, \ldots, \tau-1$. As Bergmeir et al. (2018) point out the usage for

\footnotetext{
${ }^{11}$ Previous studies such as Ferson and Siegel (2009) and Fletcher and Basu (2016) have found that timevarying conditional covariance matrix leads to marginal changes.

${ }^{12}$ For the $3 \mathrm{PRF}$, given its complexity, we pre-determined a small number of three latent factors to impose sparsity.
} 
time-series applications, we decided to make use of $K$-fold cross-validation in the training subsamples. For a list of possible different hyperparameters of each estimator (i.e., whether $\lambda, \alpha$, or the number of components $m$ ), the mean square error (MSE) is computed in this training sample. The hyperparameter(s) value(s) that produce(s) the lowest MSE is(are) chosen.

This recursion scheme allows us to incorporate the most recent data from the set of conditioning information $\mathbf{Z}$ when enlarging the in-sample period (training subsample) by one $t$ (i.e., one month), while dynamically selecting the best hyperparameter(s) for a given objective function. Finally, the observation $\tau$ is used for testing using the best-tunned model estimates for all techniques.

\subsection{Evaluating the Estimated Conditional Means}

Since we use several different approaches to impose sparsity when forming the conditional means, we are interested to assess the characteristics of the generated conditional means by each estimator. We evaluate the predictive performance for each asset $i$ using out-of-sample $R^{2}$ given by

$$
R_{i}^{2}=1-\frac{\sum_{t \in \tau_{O O S}}\left(r_{i, t+1}-\hat{r}_{i, t+1}\right)^{2}}{\sum_{t \in \tau_{O O S}}\left(r_{i, t+1}^{2}\right)}
$$

where $\tau_{O O S}$ represents the OOS periods not used for testing or tuning. Usually, the OOS $R^{2}$ is computed demeaning the denominator. As Gu et al. (2018) argues, doing so can be a mistaken analysis, since the historical averages usually underperform a naive forecast of zero. A way to overcome this issue is to compare against a benchmark of zero, as in equation (29).

It is known that the $R^{2}$ are generally extremely low when dealing with forecasting excess returns. To assess if the OOS $R^{2}$ generated by different approaches deliver economically meaningful results, we follow Campbell and Thompson (2007); Gu et al. (2018) and calculate the implied SR $\left(\mathrm{SR}^{*}\right)$. The implied SR seeks to measure the improvement produced in the $\mathrm{SR}$ when an investor can exploit signals from the conditioning information set $\mathbf{Z}$. Under mild assumptions ${ }^{13}$, the implied SR is given by

$$
S R^{*}=\sqrt{\frac{S R^{2}+R^{2}}{1-R^{2}}}
$$

The interpretation is straightforward. In the case the conditioning information produces

\footnotetext{
${ }^{13}$ Assuming an investor with a mean-variance preferences, single-period horizon, and $\gamma$ risk aversion coefficient.
} 
valuable signal, the OOS $R^{2}$ is large relative to $S R^{2}$, so the investor can exploit the information in $\mathbf{Z}$ to obtain a large proportional increase in portfolio return.

\subsection{Datasets}

As it is standard in this literature, we use size- and value- sorted and grouped portfolios from Ken French's website to represent our universe of risky assets of U.S. stocks. The chosen number $N$ of available portfolios are 25 and 100. In the Internet Appendix, we extend the analysis for other portfolios with a smaller number of assets. Precisely, we assess 5 Industry Portfolios and 6 portfolios formed on size and boot-to-market. Summary statistics tables for all portfolios are also presented in the Internet Appendix ${ }^{14}$.

With high-dimensional datasets and a vast number of suggested predictors, we use several instruments and split them into groups. The first group is formed by the predictive candidates used in Welch and Goyal (2007) and made available on Amit Goyal's website. We consider a set of 10 variables: book-to-market $(b / m)$, default return spread $(d f r)$, default yield spread ( $d f y)$, inflation ( $i n f l)$, long-term yield (lty), long-term rate of returns (ltr), net equity expansion (ntis), stock variance (svar), term spread (tms) and treasury bill rate $(t b l)$.

The second group makes use of the FRED-MD dataset as presented in McCracken and $\operatorname{Ng}(2016)$. The FRED-MD is a large macroeconomic database and monthly updated by the FRED ${ }^{15}$ that shares the predictive content of the Stock-Watson dataset Stock and Watson (1996)). It is a balanced panel consisting of 128 macroeconomic and financial variables. The variables are split into 8 groups: (1) output and income, (2) labor market, (3) housing, (4) consumption, orders, and inventories, (5) money and credit, (6) interest and exchange rates, (7) prices, and (8) stock market. In order to mitigate the consequences of the presence of cointegration, we apply the suggested transformations to obtain stationary time series. The transformations can be grouped in seven categories: (i) no transformation; (ii) $\Delta x_{t}$; (iii) $\Delta^{2} x_{t}$; (iv) $\log \left(x_{t}\right),(\mathrm{v}) \Delta \log \left(x_{t}\right)$, (vi) $\Delta^{2} \log \left(x_{t}\right)$, and (vii) $\Delta\left(x_{t} / x_{t-1}-1\right)^{16}$.

The third group is based on the economic policy uncertainty measure (EPU) from Baker et al. (2016) and related indexes. The EPU is an index that proxies for movements in policyrelated economic uncertainty for U.S., being based on newspaper coverage frequency. In the sense of the EPU, we also use the financial stress indicator (FSI) for the U.S from Püttmann (2018). The essence of the FSI is being an indicator of negative financial sentiment. It is based on the reporting in five major US newspapers 17 . Püttmann (2018) shows that the FSI

\footnotetext{
${ }^{14}$ Tables C.2 to C.5 in the Internet Appendix.

${ }^{15}$ https:/research.stlouisfed.org/econ/mccracken/fred-databases/

${ }^{16}$ Table C.1 in the Internet Appendix C presents the entire list of variables, groups, corresponding transformations, and sample means and standard deviations for the full sample, IS and OOS.

${ }^{17}$ Boston Globe, Chicago Tribune, Los Angeles Times, Wall Street Journal, and Washington Post.
} 
is a robust indicator, such that an increase in negative financial sentiment is followed by a fall in output, higher unemployment, lower stock market returns, and rising corporate bond spreads.

\section{Empirical Results}

\subsection{Out-of-Sample Analysis - Sharpe Ratios}

\subsubsection{Performance Evaluation}

To answer whether we can exploit signals provided by a large set of conditioning information using penalized estimators to impose sparsity or latent factors models with small numbers of factors, we first evaluate how the estimators behave with different sets of conditioning information to provide mean-standard deviation ratios of the returns in the out-of-sample data.

Table 2 shows the Sharpe ratios of the seven estimators for all three mean-variance efficient portfolio frameworks. For the 25 Portfolios formed on size and BTM, we see that only the OLS failed to deliver a significant SR for the MVATE with large sets of conditioning information. Overall we note that all the other estimators provided similar SR, while the PCR and the PLS alternate to deliver a higher ratio to the remaining estimators. Using Goyal's instruments we that OLS, 3PRF, LASSO, RIDGE, and ENet produce similar SR. As expected, OLS cannot handle large set of covariates, what makes the SR drop whenever we increase the set of conditioning information.

For the 100 Portfolios formed on size and BTM, a similar pattern stands out. OLS cannot extract efficiently information from a large set of instruments, making its SR to be considerably lower and not statistically significant (in most cases) compared to the rest of the estimators. Again, we notice that the PCR and the PLS deliver higher SR in most cases. Differently from the case with a smaller number of risky assets, we see that all estimators, except PLS and PCR, failed to extract signals and deliver statistically significant SRs when using a small set of instruments (Goyal). An important point to notice is that, in general, as we move to a high-dimensional setting, increasing the number of instruments, the dimensionality reduction approaches deliver a higher SR. 
Table 2: Out-of-Sample Sharpe ratios delivered by each estimator and set of conditioning information

\begin{tabular}{|c|c|c|c|c|c|c|c|c|c|c|c|c|}
\hline \multirow[b]{3}{*}{ Estimator } & \multicolumn{6}{|c|}{25 Portfolios Formed on Size and Book-to-Market } & \multicolumn{6}{|c|}{100 Portfolios Formed on Size and Book-to-Market } \\
\hline & \multicolumn{2}{|c|}{ CMV } & \multicolumn{2}{|c|}{ UMV } & \multicolumn{2}{|c|}{ MVATE } & \multicolumn{2}{|c|}{ CMV } & \multicolumn{2}{|c|}{ UMV } & \multicolumn{2}{|c|}{ MVATE } \\
\hline & $S R$ & $p$-val & $S R$ & $p$-val & $S R$ & $p$-val & $S R$ & $p$-val & $S R$ & $p$-val & $S R$ & $p$-val \\
\hline \multicolumn{13}{|l|}{ Panel A: Goyal } \\
\hline OLS & 0.307 & 0.000 & 0.293 & 0.000 & 0.270 & 0.000 & 0.076 & 0.221 & 0.072 & 0.245 & 0.003 & 0.962 \\
\hline $3 \mathrm{PRF}$ & 0.327 & 0.000 & 0.330 & 0.000 & 0.307 & 0.000 & 0.117 & 0.060 & 0.133 & 0.032 & 0.059 & 0.341 \\
\hline PLS & 0.307 & 0.000 & 0.322 & 0.000 & 0.300 & 0.000 & 0.245 & 0.000 & 0.241 & 0.000 & 0.279 & 0.000 \\
\hline PCR & 0.383 & 0.000 & 0.386 & 0.000 & 0.380 & 0.000 & 0.303 & 0.000 & 0.315 & 0.000 & 0.312 & 0.000 \\
\hline LASSO & 0.276 & 0.000 & 0.279 & 0.000 & 0.278 & 0.000 & 0.087 & 0.158 & 0.100 & 0.107 & 0.084 & 0.173 \\
\hline RIDGE & 0.313 & 0.000 & 0.311 & 0.000 & 0.299 & 0.000 & 0.060 & 0.333 & 0.083 & 0.178 & 0.044 & 0.472 \\
\hline ENET & 0.315 & 0.000 & 0.316 & 0.000 & 0.259 & 0.000 & 0.097 & 0.117 & 0.103 & 0.096 & 0.043 & 0.483 \\
\hline \multicolumn{13}{|c|}{ Panel B: FRED-MD } \\
\hline OLS & 0.161 & 0.010 & 0.166 & 0.008 & 0.006 & 0.924 & 0.128 & 0.040 & 0.128 & 0.040 & 0.047 & 0.446 \\
\hline $3 \mathrm{PRF}$ & 0.282 & 0.000 & 0.262 & 0.000 & 0.261 & 0.000 & 0.210 & 0.001 & 0.197 & 0.002 & 0.135 & 0.029 \\
\hline PLS & 0.296 & 0.000 & 0.299 & 0.000 & 0.285 & 0.000 & 0.350 & 0.000 & 0.346 & 0.000 & 0.230 & 0.000 \\
\hline PCR & 0.371 & 0.000 & 0.361 & 0.000 & 0.374 & 0.000 & 0.203 & 0.001 & 0.209 & 0.001 & 0.198 & 0.002 \\
\hline LASSO & 0.215 & 0.001 & 0.209 & 0.001 & 0.212 & 0.001 & 0.312 & 0.000 & 0.305 & 0.000 & 0.240 & 0.000 \\
\hline RIDGE & 0.274 & 0.000 & 0.277 & 0.000 & 0.275 & 0.000 & 0.274 & 0.000 & 0.265 & 0.000 & 0.188 & 0.003 \\
\hline ENET & 0.280 & 0.000 & 0.267 & 0.000 & 0.286 & 0.000 & 0.307 & 0.000 & 0.301 & 0.000 & 0.129 & 0.037 \\
\hline \multicolumn{13}{|c|}{ Panel C: All Instruments } \\
\hline OLS & 0.201 & 0.003 & 0.204 & 0.003 & -0.054 & 0.419 & 0.118 & 0.078 & 0.118 & 0.078 & -0.026 & 0.694 \\
\hline $3 \mathrm{PRF}$ & 0.290 & 0.000 & 0.278 & 0.000 & 0.254 & 0.000 & 0.240 & 0.000 & 0.228 & 0.000 & 0.145 & 0.020 \\
\hline PLS & 0.331 & 0.000 & 0.303 & 0.000 & 0.314 & 0.000 & 0.309 & 0.000 & 0.315 & 0.000 & 0.263 & 0.000 \\
\hline PCR & 0.307 & 0.000 & 0.293 & 0.000 & 0.310 & 0.000 & 0.269 & 0.000 & 0.272 & 0.000 & 0.271 & 0.000 \\
\hline LASSO & 0.231 & 0.000 & 0.230 & 0.000 & 0.232 & 0.000 & 0.323 & 0.000 & 0.315 & 0.000 & 0.253 & 0.000 \\
\hline RIDGE & 0.305 & 0.000 & 0.306 & 0.000 & 0.299 & 0.000 & 0.243 & 0.000 & 0.247 & 0.000 & 0.117 & 0.079 \\
\hline ENET & 0.302 & 0.000 & 0.280 & 0.000 & 0.302 & 0.000 & 0.359 & 0.000 & 0.352 & 0.000 & 0.179 & 0.008 \\
\hline
\end{tabular}

Table 2 summarises the OOS (Jan-1996 to Dec-2017) Sharpe ratios (SR) by estimator and optimal portfolio framework (CMV, UMV and MVATE) for both portfolios. Panel A reports the Sharpe ratios generated when the variables from Goyal's website are used as Z. Goyal variables comprises: $b / m, d f r$, dfy, infl, ltr, lty, ntis, svar, tms and tbl. Panel B presents the Sharpe ratios obtained using the FRED-MD variables. The FRED-MD is a large dataset containing 128 macroeconomic and financial variables. Finally, panel C shows the Sharpe rations when all variables are used as conditioning information. "All Instruments" is the combination of Goyal and FRED-MD datasets with the Economic Policy Uncertainty (EPU) index and the Financial Stress Indicator (FSI). The $p$-val is the p-value from the two-sided test of the SR. 


\subsubsection{Statistical Inference for the Difference of Sharpe Ratios}

To answer if the Sharpe ratios generated by an estimator is statistically different from another SR generated by any other estimator, we need to test the difference between them. We follow Ledoit and Wolf (2008) to perform these tests. In short, consider the Sharpe ratios of the optimal portfolios generated by two different estimators, say a and b. The difference between $S R_{a}$ and $S R_{b}$ is given by

$$
\Delta=S R^{(a)}-S R^{(b)}=\frac{\mu^{(a)}}{\sigma^{(a)}}-\frac{\mu^{(b)}}{\sigma^{(b)}}
$$

where $\hat{\mu}$ and $\hat{\sigma}$ are the OOS unconditional mean and variance of the excess returns from the optimal portfolio generated by an estimator. Ledoit and Wolf (2008) suggest to construct a studentized time series bootstrap confidence interval for the difference of the SRs. This method has been shown to be robust when returns have tails heavier than the normal distribution or are of time series nature. The bootstrap data is generated using the circular block bootstrap of Politis and Romano (1992). The two-sided distribution function of the studentized statistic can be obtained via bootstrap as follows:

$$
f\left(\frac{|\widehat{\Delta}-\Delta|}{s e(\widehat{\Delta})}\right) \approx f\left(\frac{\left|\widehat{\Delta}^{\text {boot }}-\Delta\right|}{s e\left(\widehat{\Delta}^{\text {boot }}\right)}\right)
$$

where $f(\cdot)$ is the distribution of a random variable, $\Delta$ is populational difference between $S R_{a}$ and $S R_{b}, \widehat{\Delta}$ is the sample counterpart of this difference obtained in the data in the estimation window, and $\widehat{\Delta}^{\text {boot }}$ is the estimated difference computed from bootstrap. The standard errors are denoted by $s e(\cdot)$. Out of the distribution obtained from the bootstrap in the equation $(32)$, we can find the confidence interval for $\Delta$ and the p-values of the test ${ }^{18}$.

Table 3 reports for the dataset with 100 portfolios formed on Size/BTM. Table 4 presents the test of difference of SRs for the dataset with 25 portfolios formed on Size/BTM[19. In order to see that the Sharpe ratios generated using not only two different estimators, but also different set of conditioning information, these tables also present the tests of difference between these cases too.

Inspecting table 3, we notice that in the case with 100 risky assets PLS and PCR indeed generate different SRs to all the other 5 estimators when using the Goyal variables as conditioning information. The p-values of the difference of Sharpe ratios are always low, providing a strong evidence to this difference. This fact is valid for all three mean-variance

\footnotetext{
${ }^{18}$ For further details in this procedure, see Ledoit and Wolf $(2008)$.

${ }^{19}$ In the Internet Appendix, tables B.2 and table B.3 report the same tests for the dataset with 6 portfolios formed on Size/BTM and the dataset with 5 industry portfolios respectively.
} 
Table 3: Test for the difference of the Sharpe ratios - OOS (Jan-1996 - Dec-2017) - 100 Portfolios

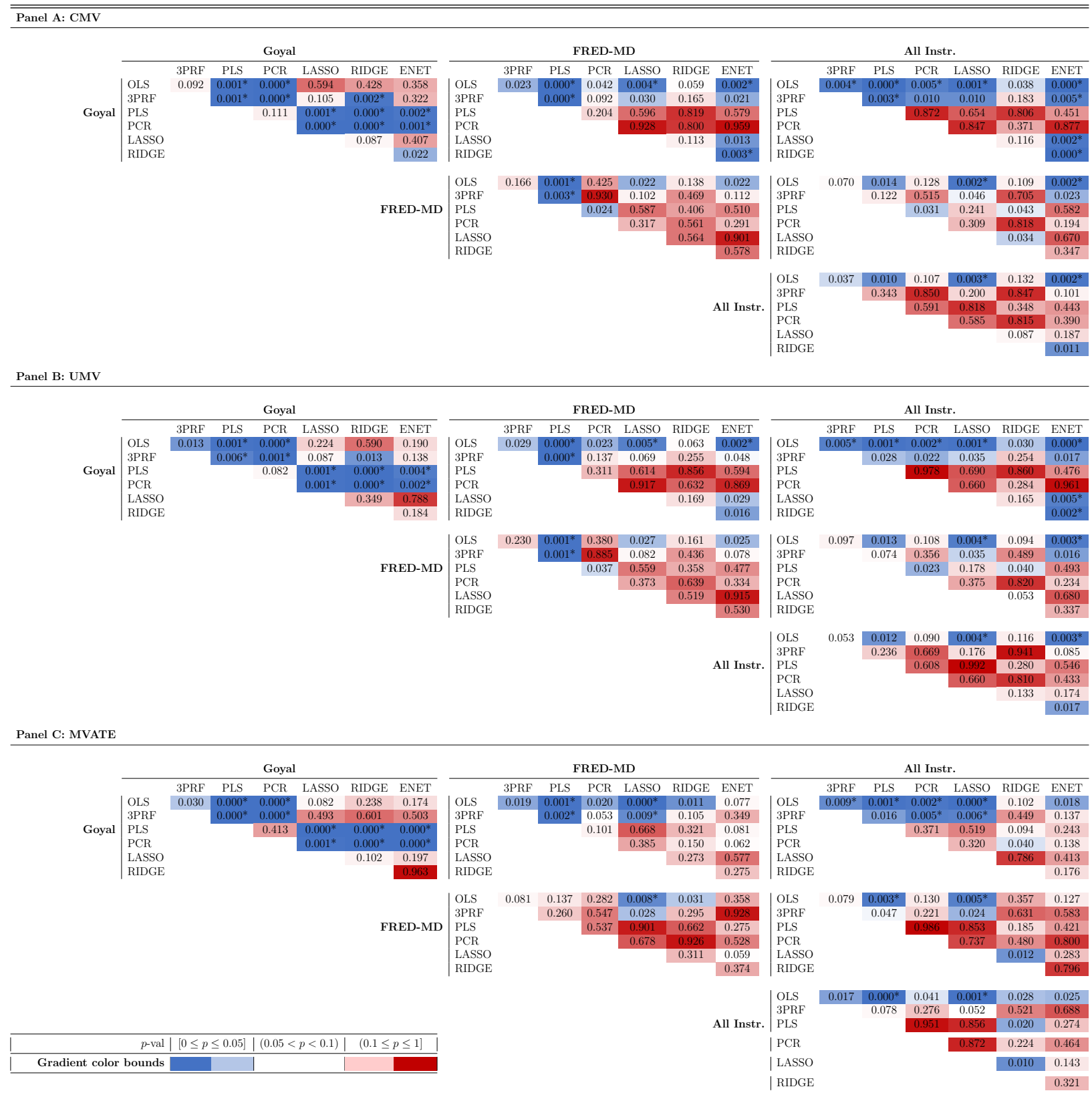

Test for the differences of the Sharpe ratios of the OOS (Jan-1996 - Dec-2017) returns of the efficient portfolios formed from the dataset with 100 Size/BTM portfolios using 7 different estimators (OLS, 3PRF, PLS, PCR, LASSO, RIDGE and ENet) and three different set of conditioning information(Goyal, FRED-MD and "All Instruments"). Each panel shows the test of pairs of Sharpe ratios for three different framework to build efficient portfolios. Panel A reports conditionally mean-variance (CMV) efficient portfolios. Panel B reports unconditionally mean-variance efficient portfolios. Panel C presents the mean-variance tracking error (MVATE) portfolios. We split the results depending on conditioning information set used. Goyal variables comprises: $b / m$, $d f r$, dfy, infl, ltr, lty, ntis, svar, tms and $t b l$. The FRED-MD is a large dataset containing 128 macroeconomic and financial variables. Finally, "All Instruments" is the combination of Goyal and FRED-MD datasets with the Economic Policy Uncertainty (EPU) index and the Financial Stress Indicator (FSI). 
Table 4: Test for the difference of the Sharpe ratios - OOS (Jan-1996 - Dec-2017) - 25 Portfolios
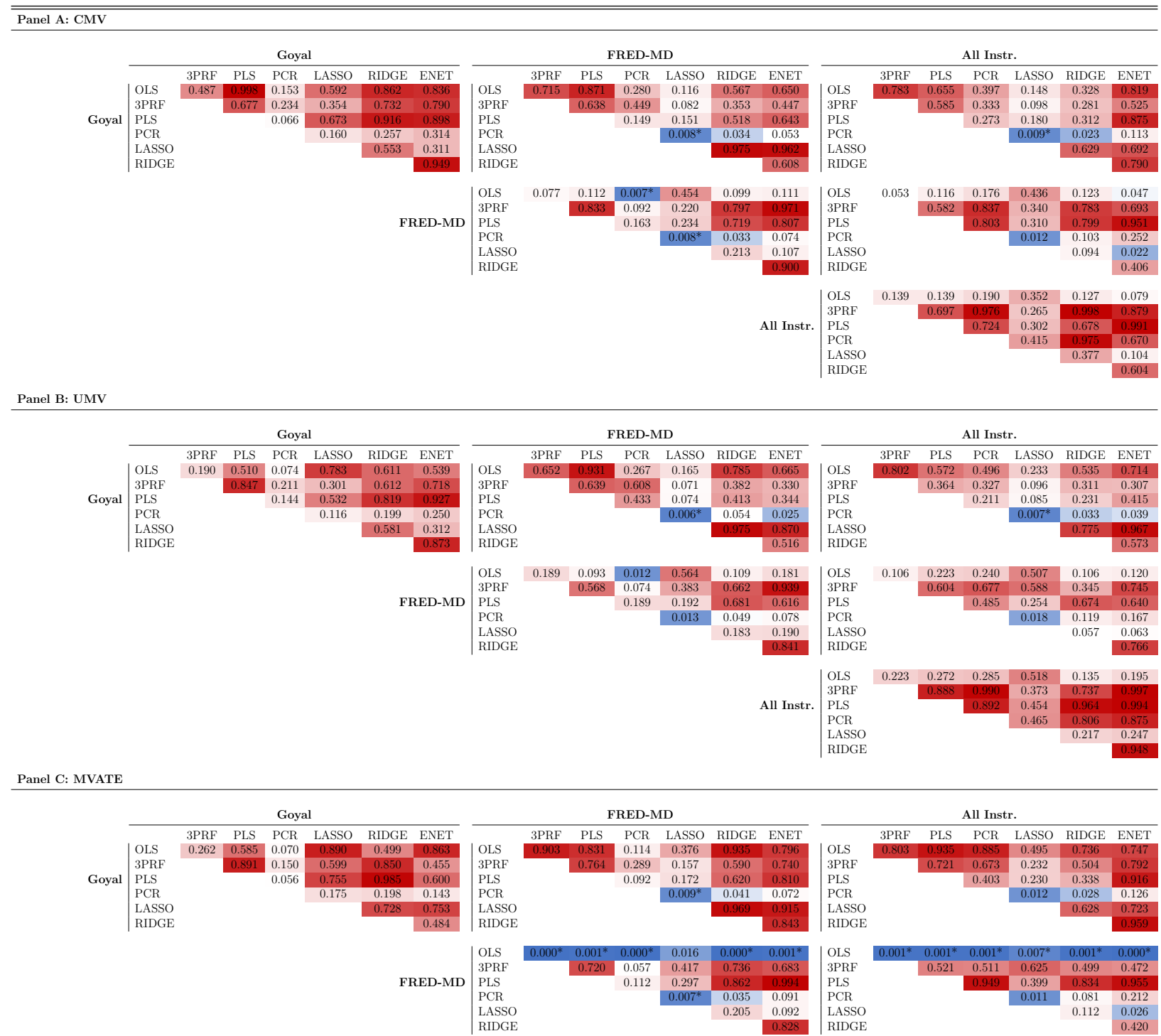

All Instr.

OLS
3PRF

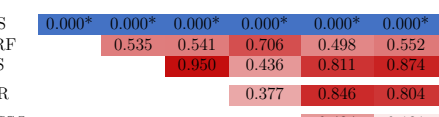

LASSO

RIDGE

$\begin{array}{lll}0.434 & 0.131\end{array}$

Gradient color bounds

Test for the differences of the Sharpe ratios of the OOS (Jan-1996 - Dec-2017) returns of the efficient portfolios formed from the dataset with 25 Size/BTM portfolios using 7 different estimators (OLS, 3PRF, PLS, PCR, LASSO, RIDGE and ENet) and three different set of conditioning information(Goyal, FRED-MD and "All Instruments"). Each panel shows the test of pairs of Sharpe ratios for three different framework to build efficient portfolios. Panel A reports conditionally mean-variance (CMV) efficient portfolios. Panel B reports unconditionally mean-variance efficient portfolios. Panel C presents the mean-variance tracking error (MVATE) portfolios. We split the results depending on conditioning information set used. Goyal variables comprises: $b / m, d f r, d f y$, infl, ltr, lty, ntis, svar, tms and $t b l$. The FRED-MD is a large dataset containing 128 macroeconomic and financial variables. Finally, "All Instruments" is the combination of Goyal and FRED-MD datasets with the Economic Policy Uncertainty (EPU) index and the Financial Stress Indicator (FSI). 
framework. Combined with the results from table 2, we can claim that the SRs of 0.245, 0.241 and 0.279 (CMV, UMV, MVATE respectively) for the PLS, and the SRs of 0.303, 0.315 and 0.312 (CMV, UMV, MVATE respectively) produced by the PCR are indeed higher than the remaining ones.

\subsection{Characteristics and the Distribution of Optimized Portfolio Weights}

In order to understand why a specific estimator can generate mean-variance optimized portfolio with higher return-volatility ratios we first analyze how the weights of the optimized portfolio behave for each estimator and set of conditioning information. For each month $t$ in the out-of-sample period, each estimator produced a set of weights depending on the matrix $\mathbf{Z}$ used. We pooled the $N$ weights for each $t$ and plotted the distribution of these weights in separate figures for each dataset employed. Figure 1 plots the weights for the dataset with 25 portfolios formed on Size/BTM. Figure 2 shows the distribution of the weights $\mathbf{x}_{t}\left(\mathbf{Z}_{t}\right)$ for the dataset with 100 portfolios formed on Size/BTM 20 . Overall we see that across all three efficient strategies to build portfolios, PCR generates weights with higher variance than compared to the other estimators. Another point that can be inferred from these figures is that, in general, most of the estimators generate symmetric weights distributions. However, PCR does not, having in most cases a right-skewed distribution. We also notice that OLS is the estimator that does not respond much to predictive information, a fact that can be seen from its highly concentrated distribution around zero.

\footnotetext{
${ }^{20}$ In the Internet Appendix, we present the weights for the dataset with 6 portfolios formed on Size/BTM and the dataset with 5 industry portfolios.
} 
Figure 1: Distribution of Optimized Portfolio Weights - 25 Portfolios Formed on Size and Book-to-Market
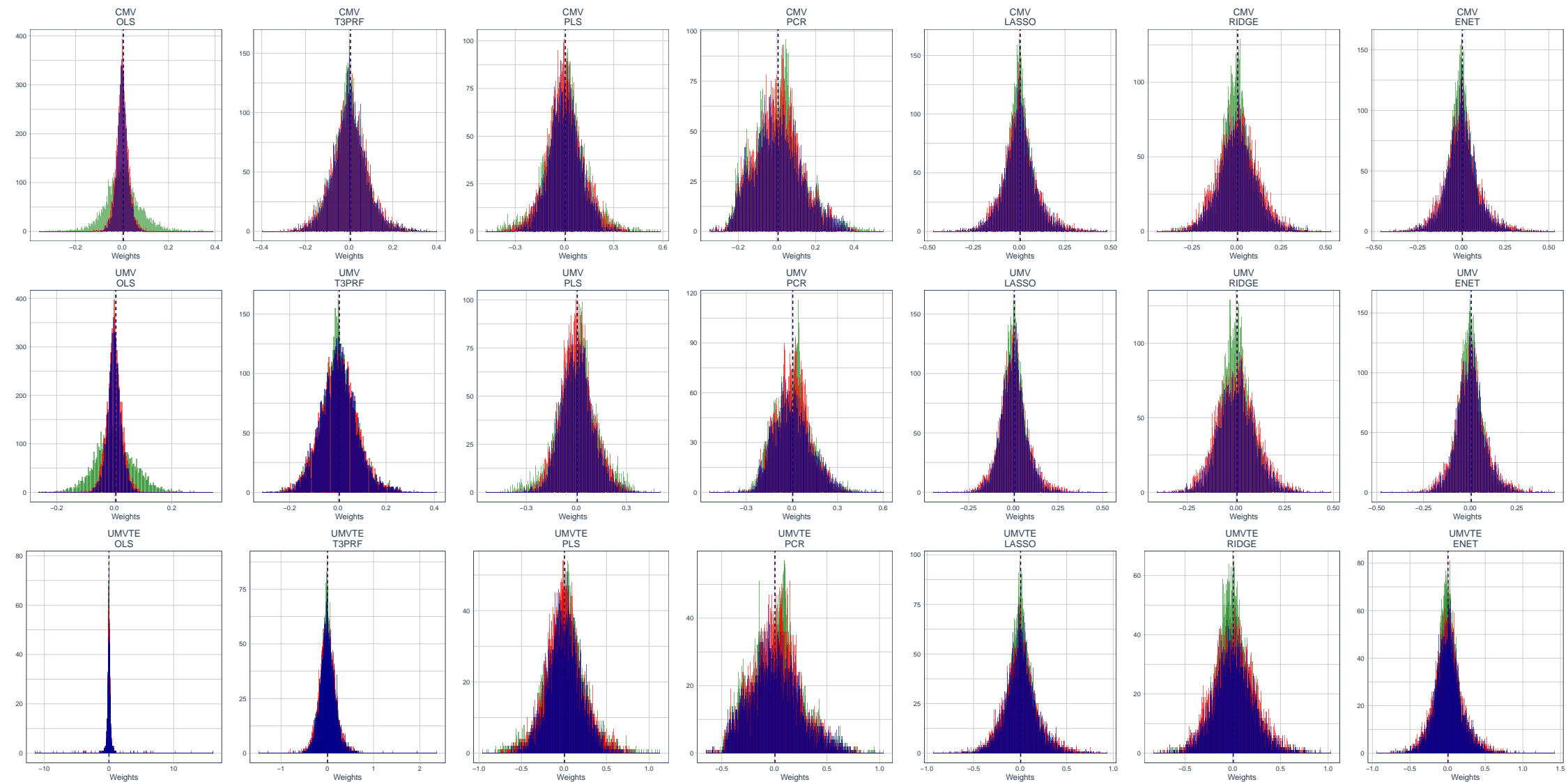

Figure 1 plots the distribution of the optimized portfolios weights generated by each estimator (columns) and mean-variance optimal framework (rows) for the 25 portfolios formed on Size/BTM. The first row reports the CMV strategies, the second row reports the UMV, and the third one plots the UMVTE. The set of conditioning information used in $\mathbf{Z}$ are plotted in different colors: (i) Goyal's in green, (ii) FRED-MD in red, and (iii) "All Instruments" in blue. Goyal's variables comprises: $b / m$, dfr, dfy, infl, ltr, lty, ntis, svar, tms and tbl. FRED-MD is a large dataset containing 128 macroeconomic and financial variables. "All Instruments" is the combination of Goyal and FRED-MD datasets with the Economic Policy Uncertainty (EPU) index and the Financial Stress Indicator (FSI). 
Figure 2: Distribution of Optimized Portfolio Weights - 100 Portfolios Formed on Size and Book-to-Market
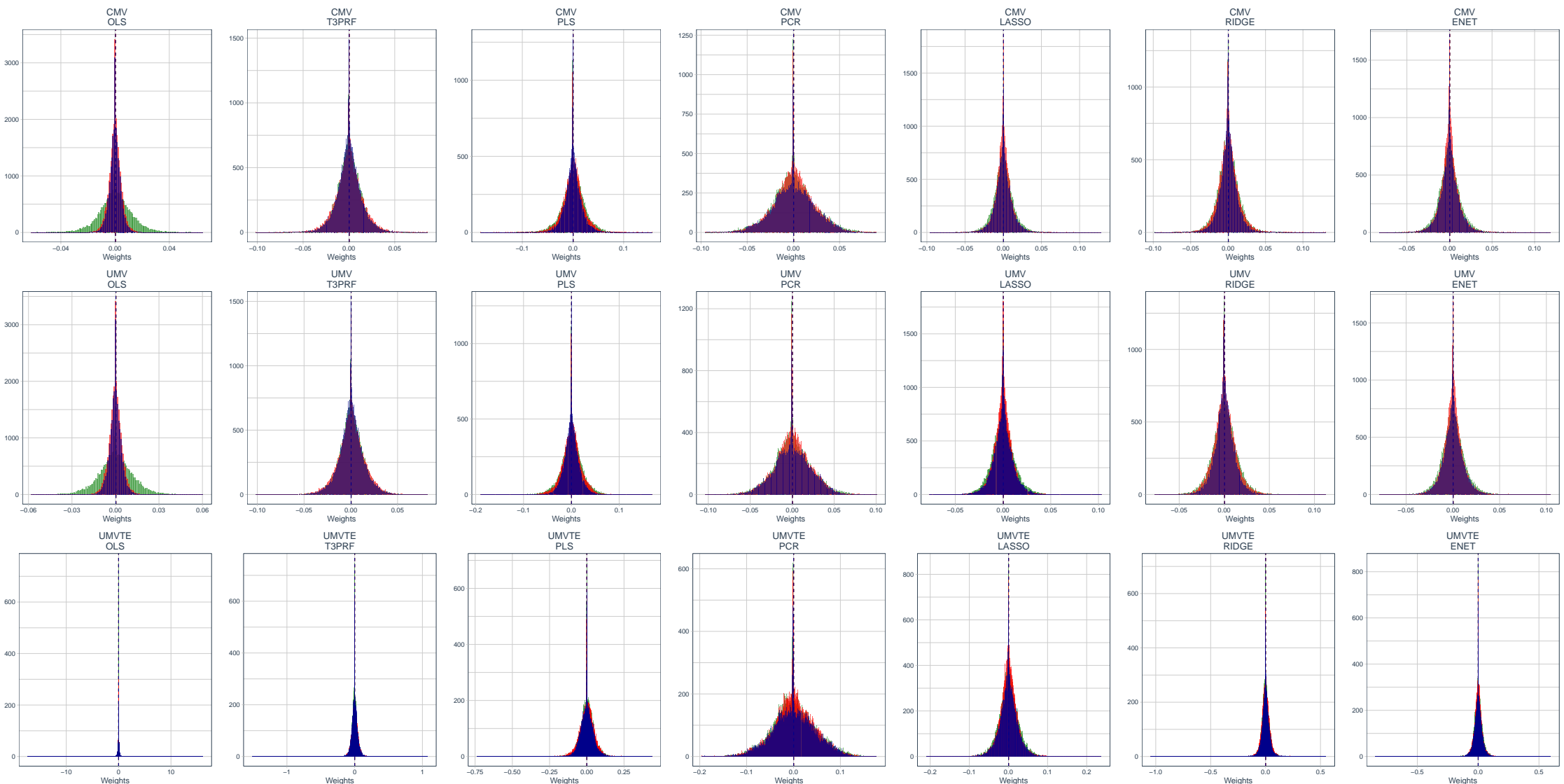

Figure 2 plots the distribution of the optimized portfolios weights generated by each estimator (columns) and mean-variance optimal framework (rows) for the 100 portfolios formed on Size/BTM. The first row reports the CMV strategies, the second row reports the UMV, and the third one plots the UMVTE. The set of conditioning information used in $\mathbf{Z}$ are plotted in different colors: (i) Goyal's in green, (ii) FRED-MD in red, and (iii) "All Instruments" in blue. Goyal's variables comprises: $b / m$, dfr, dfy, infl, ltr, lty, ntis, svar, tms and tbl. FRED-MD is a large dataset containing 128 macroeconomic and financial variables. "All Instruments" is the combination of Goyal and FRED-MD datasets with the Economic Policy Uncertainty (EPU) index and the Financial Stress Indicator (FSI). 


\subsection{Variable Contribution}

The results from the previous sections raise the natural question of what variables are important to each estimator. To answer this question we evaluate how each lagged variable contributed to produce the estimated conditional means in the OOS. After we obtain the estimates of the conditional mean $\widehat{\mu}_{i}\left(\mathbf{Z}_{t-1}\right)=\widehat{\mathbb{E}}\left(r_{i, t} \mid \mathbf{Z}_{t-1}\right)$ for each risky asset $i=1, \ldots, N$ at each $t$, we evaluate how each lagged variable in our sets of conditioning information contribute to generate $\widehat{\boldsymbol{\mu}}_{t}$ to be used to form the weights in $\mathbf{x}_{t}\left(\mathbf{Z}_{t}\right)$.

We take a straightforward approach to assess this contribution. At each month $t$, we compute the absolute values of the estimated coefficients in equation (6) for each asset $i$ and lagged variable $k$ in the conditioning set. For the estimators that we standardized the instruments before the regression, we multiply each $\widehat{\theta}_{i, l}$ by its own standard deviation computed in the estimation window. Grouping by estimators and $\mathbf{Z}$ used, we pool all the estimates of the $N$ assets and calculate the average of the absolute values. In order to make the comparison clear, we normalize these means to sum one. Doing so, we can rank the most influential covariates driving the conditional means on a percentage scale.

Figure 3 reports for the dataset with 100 portfolios formed on size and BTM the 10 most influential lagged variables for all the estimator:21. Interestingly, the PLS and PCR have some similarities regarding variable contribution, however this is not consistent across all three sets of conditioning information. Using FRED-MD, PLS and PCR ranked similarly the variables. The five most influential are: $H W I$ (Help-Wanted Index for US), AAAFFM (Moody's Aaa Corporate Bond minus FEDFUNDS Aaa-FF spread), BAAFFM (Moody's Baa Corporate Bond minus FEDFUNDS Baa-FF spread), T10YFFM (10-Year Treasury C Minus FEDFUNDS 10 yr-FF spread), T5YMFFM (5-Year Treasury C minus FEDFUNDS 5 yr-FF spread) for both estimators. On the other hand, using all conditioning information, there is a clear difference in weights. The majority of the influence for PCR is split by two variables $(A A A F F M$ and $B A A F F M)$, while for PLS these two variables respond less than $30 \%$, with a labor market variable $(H W I)$ also having a large impact (35\%), and small contributions from interest and exchange rates variables (T1YFFM, TB6SMFFM, TB3SMFFM). Out of the ten variables from Goyal's dataset, only three contributes to build efficient portfolios when using PCR (tbl, lty and tms).

For LASSO, Ridge, and Enet we see a similar pattern for all three estimators depending on which set of conditioning information was used. Finally, for OLS we see a completely different pattern compared to the previous estimators. We notice that labor market variables such as DMANEMP (All Employees: Durable goods), NDMANEMP (All Employees: Nondurable

\footnotetext{
${ }^{21}$ Except for the 3PRF which is a latent factor model
} 
Figure 3: Variable Contribution by Estimator and Set of Conditioning Information - 100 Portfolios Formed on Size and Book-to-Market
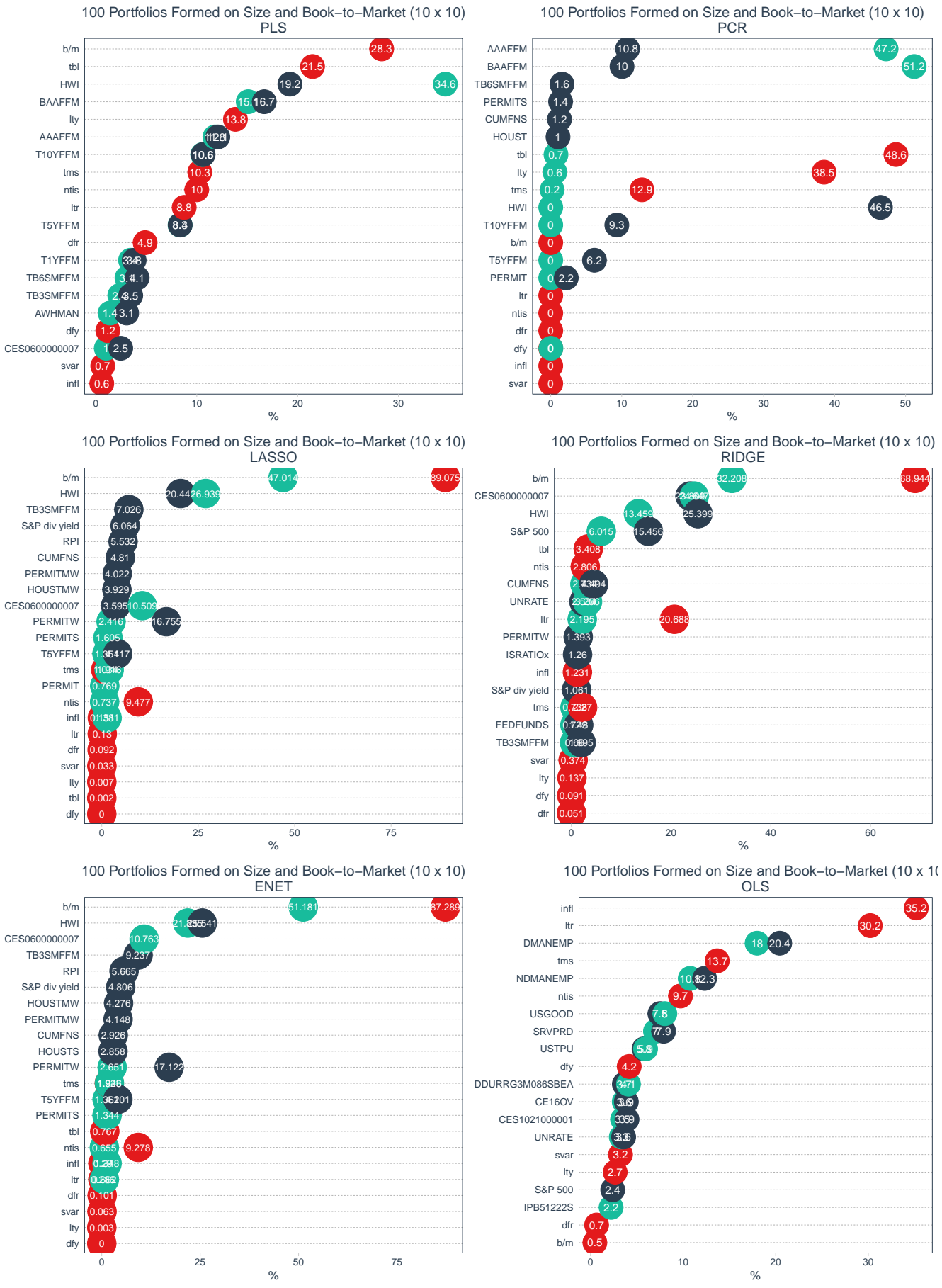

Figure 3 reports the 10 most influential variables by estimator (panels) and set of conditioning information used (colors) for the for the 100 portfolios formed on Size/BTM. We take a direct approach to obtain the contribution for each variable. At each month $t$, we compute the absolute values of the estimated coefficients in (6) for each asset $i$ and lagged variable $k$ in the conditioning set. We destandardize the variables whenever necessary. Grouping by estimators and $\mathbf{Z}$ used, we pool all the estimates of the $N$ assets and calculate the average of the absolute values. In order to make the comparison clear, we normalize these means to sum one. 
Figure 4: Variable Contribution by Estimator and Set of Conditioning Information - 25 Portfolios Formed on Size and Book-to-Market
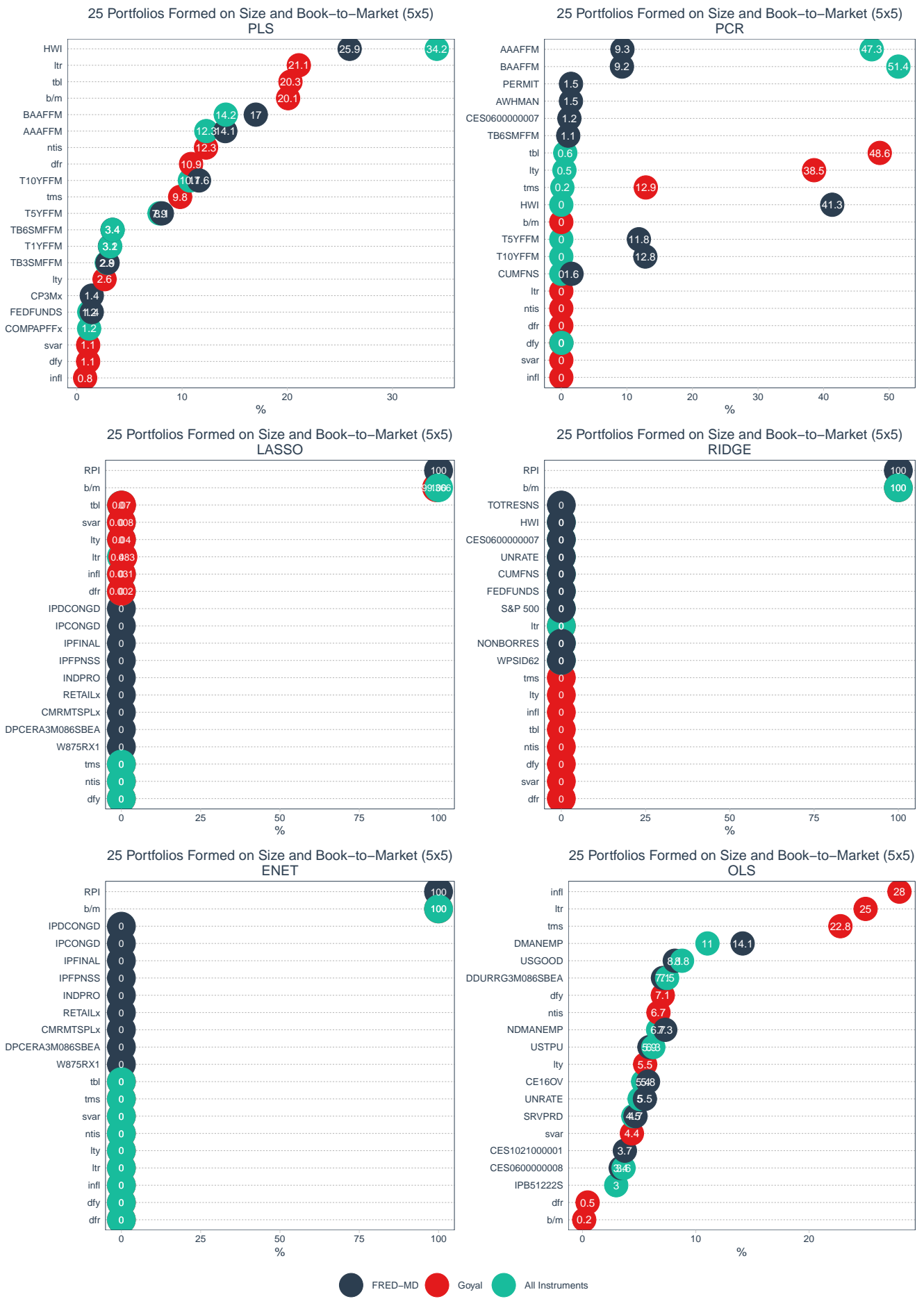

Figure 4 reports the 10 most influential variables by estimator (panels) and set of conditioning information used (colors) for the for the 25 portfolios formed on Size/BTM. We take a direct approach to obtain the contribution for each variable. At each month $t$, we compute the absolute values of the estimated coefficients in (6) for each asset $i$ and lagged variable $k$ in the conditioning set. We destandardize the variables whenever necessary. Grouping by estimators and $\mathbf{Z}$ used, we pool all the estimates of the $N$ assets and calculate the average of the absolute values. In order to make the comparison clear, we normalize these means to sum one. 
goods) and USGOOD (All Employees: Goods-Producing Industries) have a large impact when using large sets of predictors. These differences may provide us information on why some estimators produced better OOS mean-variance ratios.

Figure 4 illustrates the same analysis for the dataset with 25 portfolios formed on size and $\mathrm{BTM}^{22}$, The pattern for PCR and PLS is remarkably similar as seen for 100 portfolios formed on size and BTM. The same can be said regarding the OLS been driven mostly by labor market variables with high-dimensional conditioning information sets. However, for the penalized estimators we see a different story. We see that LASSO, Ridge, and ENet did a large shrinkage and selection in the set of lagged variables. For each one, the conditioning information set practically one variable was responsible for the full contribution: an output and income variable, RPI (Real Personal Income) using FRED-MD, and $b / m$ (book-tomarket) using Goyal and a combination of all lagged instruments.

\subsection{Out-of-Sample Analysis - The Behavior of the Conditional Mean Estimates}

To form the weights of the optimized portfolios, many factors could play a role to drive the formation of mean-variance efficient portfolios. Being the conditional mean one of these components, how the estimates behave? In order to answer this question, one way to evaluate the quality of the estimates is to assess the predictive performance of each estimator when using each set of lagged variables. Following Gu et al. (2018); Kelly et al. (2019) we calculate the out-of-sample $R^{2}$ as given in equation 29 for all 7 estimators and three different sets of conditioning information for each $\tau \in \tau_{O O S}$. Given the large number of result:23, we present them in different figures.

Figure 5 reports the OOS $R^{2}$ for the 100 and 25 portfolios formed on Size/BTM ${ }^{24}$. It is clear how PLS and PCR generate higher OOS $R^{2}$ compared to most of the estimators. This result is consistent across different sets of conditioning information and sets of risky assets. The figure also reports the pooled ${ }^{25}$ OOS $R^{2}$, in which we pooled all risky assets and calculated the generated total OOS pooled $R^{2}$. In general, we see that again PLS and PCR

\footnotetext{
${ }^{22}$ In the Internet Appendix, we present similar analysis for the dataset with 6 portfolios formed on Size/BTM and the dataset with 5 industry portfolios.

${ }^{23}$ One for each asset $i=1,2, \ldots, N$, estimator $(7)$, and set of lagged variables (3).

${ }^{24}$ In the Internet Appendix, we present similar analysis for the dataset with 6 portfolios formed on Size/BTM and the dataset with 5 industry portfolios respectively.

25

$$
R_{i, t}^{2^{\text {(pooled) }}}=1-\frac{\sum_{i=1}^{N} \sum_{t \in \tau_{O O S}}\left(r_{i, t+1}-\hat{r}_{i, t+1}\right)^{2}}{\sum_{i=1}^{N} \sum_{t \in \tau_{O O S}}\left(r_{i, t+1}^{2}\right)}
$$


Figure 5: Out-of-Sample $R^{2}-100$ and 25 Portfolios Formed on Size and Book-to-Market
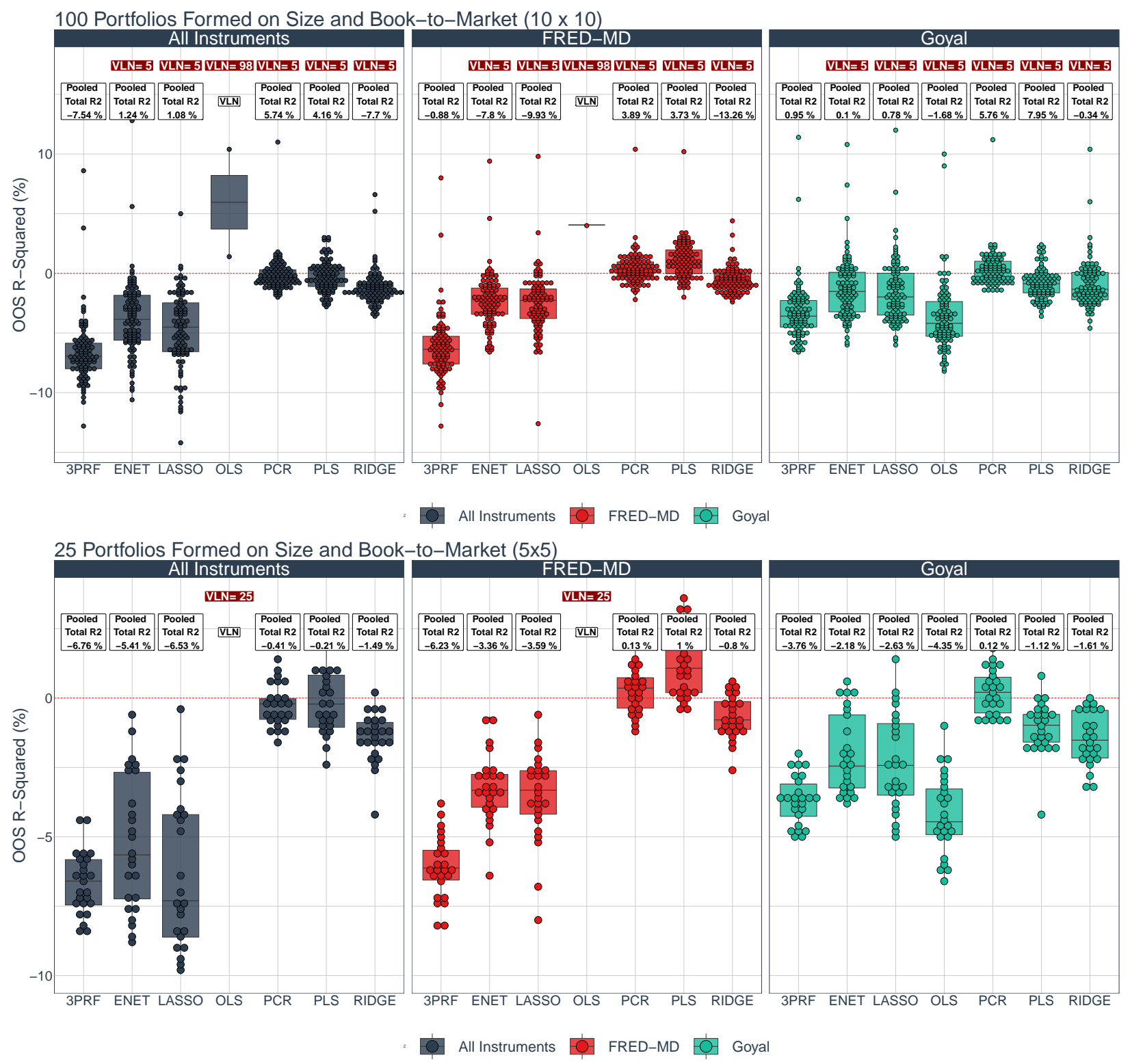

Figure 5 reports the OOS $R^{2}$ for the datasets with 100 and 25 portfolios formed on Size/BTM by estimator and set of conditioning information used, along with overlapping boxplots. The pooled OOS $R^{2}$ across all $N$ risky assets in each dataset is also reported in a white box on the top of each plot. In order to make the plots readable and comparable, we filtered the OOS $R^{2}$ larger than -0.5 in absolute values and present the amount of assets that generated these very large numbers (VLN). The amount of VLN per estimator is reported in a red box on top of each plot. 
can generate higher and in most cases positive $R^{2}$. Recall that these are monthly returns, and therefore positive $R^{2}$, even small ones, has a meaningful economic impact.

\subsection{Economic Value}

As argued in section 4.2, we can use the concept from Campbell and Thompson (2007) to evaluate how different estimators, using different predictive signals, can deliver economically meaningful results. Table 5 reports the implied Sharpe ratios for an investor with meanvariances preferences exploiting predictive information from the conditioning set. It is clear that PLS and PCR deliver higher monthly implied Sharpe ratios. Notice that when the OOS $R^{2}$ is negative, larger absolute $R^{2}$ values than the Sharpe ratio will cause the impossibility of the implied Sharpe ratio to be calculated.

\subsubsection{Economic Gains}

We use two measures to capture possible economic gains produced for optimal portfolios generated using high-dimensional data imposing sparsity in the different estimators. First, we consider the certainty equivalent return (CER) excess return of each optimal portfolio, which can be calculated as

$$
\mathrm{CER}=\hat{\mu}_{p}-\frac{\gamma}{2} \hat{\sigma}_{p}
$$

where $\hat{\mu}_{p}$ and $\hat{\sigma}_{p}$ are the OOS unconditional mean and variance of the excess returns from the optimal portfolio generated by an estimator, $\gamma$ is the risk aversion coefficient. We follow Brandt (2010); Goto and Xu (2015); Fletcher and Basu (2016) and set $\gamma$ equal to 5. Higher CER values for a given optimal portfolio can be seen as that this portfolio has a better risk-return characteristic.

Table 6 reports the monthly CER in percentage for all cases. Inside brackets, we also report the ranking among all 7 estimators within strategy and set of conditioning information employed. We see that PLS and PCR generate a higher CER for an investor with risk aversion coefficient $\gamma=5$ when $N$ available risky assets is large (either 25 or 100) ${ }^{26}$, For 25 portfolios formed on Size/BTM we see that Ridge also generates high monthly CER. Overall, another fact that we can infer from the table is that the CER ranking change just marginally across the three approaches (CMV, UMV, MVATE).

Another way to infer possible economic gains of using a better approach to form meanvariance efficient portfolios is to measure the maximum fee an investor would pay to switch

\footnotetext{
${ }^{26}$ Table B.5 in the Internet Appendix presents the CER for 6 portfolios formed on Size/BTM and the dataset with 5 industry portfolios.
} 
Table 5: Implied Sharpe Ratios

\begin{tabular}{|c|c|c|c|c|c|c|}
\hline \multirow[b]{3}{*}{ Panel A: Goyal } & \multicolumn{3}{|c|}{$\begin{array}{l}25 \text { Portfolios Formed on } \\
\text { Size and Book-to-Market }\end{array}$} & \multicolumn{3}{|c|}{$\begin{array}{l}100 \text { Portfolios Formed on } \\
\text { Size and Book-to-Market }\end{array}$} \\
\hline & CMV & UMV & MVATE & CMV & UMV & MVATE \\
\hline & & & & & & \\
\hline OLS & 0.220 & 0.202 & 0.167 & - & - & - \\
\hline $3 \mathrm{PRF}$ & 0.258 & 0.262 & 0.234 & 0.153 & 0.166 & 0.115 \\
\hline PLS & 0.286 & 0.302 & 0.279 & 0.389 & 0.387 & 0.413 \\
\hline PCR & 0.384 & 0.388 & 0.382 & 0.398 & 0.408 & 0.406 \\
\hline LASSO & 0.221 & 0.225 & 0.223 & 0.125 & 0.134 & 0.122 \\
\hline RIDGE & 0.284 & 0.281 & 0.268 & 0.014 & 0.060 & - \\
\hline ENET & 0.275 & 0.277 & 0.211 & 0.102 & 0.108 & 0.053 \\
\hline
\end{tabular}

Panel B: FRED-MD

\begin{tabular}{lcccccc}
\hline OLS & - & - & - & - & - & - \\
3PRF & 0.128 & 0.076 & 0.074 & 0.187 & 0.173 & 0.097 \\
PLS & 0.314 & 0.317 & 0.304 & 0.407 & 0.404 & 0.306 \\
PCR & 0.373 & 0.363 & 0.376 & 0.289 & 0.293 & 0.285 \\
LASSO & 0.099 & 0.086 & 0.094 & - & - & - \\
RIDGE & 0.258 & 0.261 & 0.259 & - & - & - \\
ENET & 0.208 & 0.191 & 0.216 & 0.123 & 0.109 & -
\end{tabular}

Panel C: All Instruments

\begin{tabular}{lcccccc}
\hline OLS & - & - & - & - & - & - \\
3PRF & 0.125 & 0.096 & - & - & - & - \\
PLS & 0.327 & 0.300 & 0.311 & 0.378 & 0.383 & 0.340 \\
PCR & 0.299 & 0.285 & 0.303 & 0.371 & 0.374 & 0.372 \\
LASSO & - & - & - & 0.341 & 0.333 & 0.275 \\
RIDGE & 0.278 & 0.279 & 0.271 & - & - & - \\
ENET & 0.187 & 0.152 & 0.187 & 0.378 & 0.371 & 0.212 \\
\hline \hline
\end{tabular}

Table 5 reports the implied $S R^{*}$ as given in equation $\sqrt{30}$ for all seven estimators (OLS, 3PRF, PLS, PCR, LASSO, Ridge and ENet), three different sets of conditioning information (Goyal's, FRED-MD, and "All Instruments", wich is the combination of the previous two with the Economic Policy Uncertainty (EPU) index and the Financial Stress Indicator (FSI) ) and three different mean-variance approaches (CMV, UMV, MVATE) to build efficient portfolios. Notice that when the OOS $R^{2}$ is negative, larger absolute $R^{2}$ values than the Sharpe ratio will cause the impossibility of the implied Sharpe ratio to be calculated. These cases are omitted in the table. 
Table 6: Certainty Equivalent Excess Returns (CER) (Monthly \%)

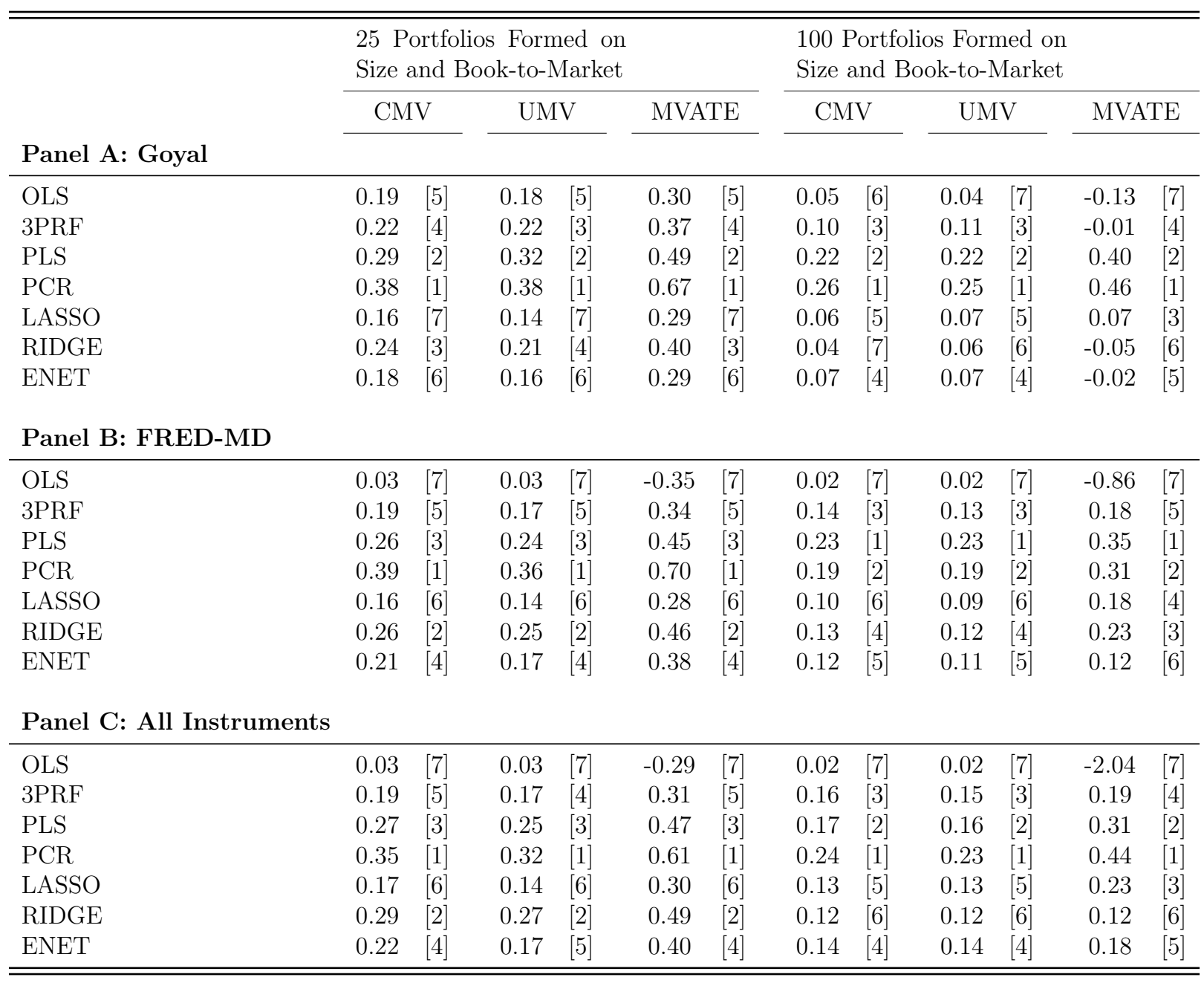

Table 6 summarises the CER (monthly \%) by estimator and optimal portfolio framework (CMV, UMV and MVATE) for both portfolios. Panel A reports the Sharpe ratios generated when the variables from Goyal's website are used as $\mathbf{Z}$. Goyal variables comprises: $b / m$, dfr, dfy, infl, ltr, lty, ntis, svar, tms and tbl. Panel B presents the Sharpe ratios obtained using the FRED-MD variables. The FRED-MD is a large dataset containing 128 macroeconomic and financial variables. Finally, panel C shows the Sharpe rations when all variables are used as conditioning information. "All Instruments" is the combination of Goyal and FREDMD datasets with the Economic Policy Uncertainty (EPU) index and the Financial Stress Indicator (FSI). Inside brackets, we also report the ranking among all 7 estimators within strategy and set of conditioning information employed 
from one approach to another. This management fee, motivated by Fleming et al. (2001), assumes a risk-averse investor with preferences given by a quadratic von Neumann-Morgenstern utility function 27 . This fee can be obtained by solving the following problem:

$$
\mathbb{E}\left(r_{p, t}^{(a)}-\mathcal{F}\right)-\frac{\gamma}{2(1+\gamma)} \mathbb{E}\left(\left(r_{p, t}^{(a)}-\mathcal{F}\right)^{2}\right)=\mathbb{E}\left(r_{p, t}^{(b)}\right)-\frac{\gamma}{2(1+\gamma)} \mathbb{E}\left(r_{p, t}^{2^{(b)}}\right)
$$

where $r_{p, t}^{(a)}$ is the OOS optimal portfolio return generated by a strategy making use of estimator $(a)$, while $r_{p, t}^{(b)}$ is the benchmark OOS optimal portfolio return generated by estimator (b). Solving for $\mathcal{F}$ we can find the management fee that an investor would be willing to pay to have access to a better formation of an optimal portfolio.

Figure 6 reports the management fee, computed as the solution for $\mathcal{F}$ in equation (36). The comparison is made in pairs, assessing the management fee generated switching from $(a)$ to $(b)$. We can interpret the results in this figure, as the management fee of switching from a strategy given in the vertical axis, which shows the combination of the estimator employed and the mean-variance approach used, to another one plotted in different colors for all seven techniques and set of conditioning information. Essentially, we see that a positive management fee is generated when switching to either PLS or PCR. There are few cases in which switching from a penalized estimator to PLS or PCR produces a negative management fee.

\subsection{Out-of-Sample Analysis - Portfolio Efficiency}

Can these dimensionality reduction techniques generate significant alphas in a standard factor model? We answer this question in this section. We evaluate how the returns of the optimal portfolios produced by all seven estimators behave when evaluated in a standard factor model to explain the risk premia. We compare all possible combinations in the Fama-French three- and five-factor models, as well as with a Fama-French five-factor with momentum. The p-values of the generated alphas are plotted in figure 7, while the $R^{2}$ of the regressions are presented in figure 8. It is clear that in most cases, for $N$ large PLS and PCR can generate statistically significant alphas. Table 7 present more details from the regression, reporting the alphas, $t$-statistics, $p$-values and the $R^{2}$ of the regressions using only the FRED-MD as the set of lagged instruments. In the Internet Appendix, we present the

${ }^{27}$ The expected utility function from the risk-averse investor is given by

$$
U=W_{0}\left(\mathbb{E}\left(r_{p, t}\right)-\frac{\gamma}{2(1+\gamma)} \mathbb{E}\left(r_{p, t}\right)^{2}\right)
$$

where $W_{0}$ represents the invertor's initial wealth, $\gamma$ a constant risk aversion coefficient, and $r_{p, t}$ the portfolio return. 
Figure 6: Management Fee

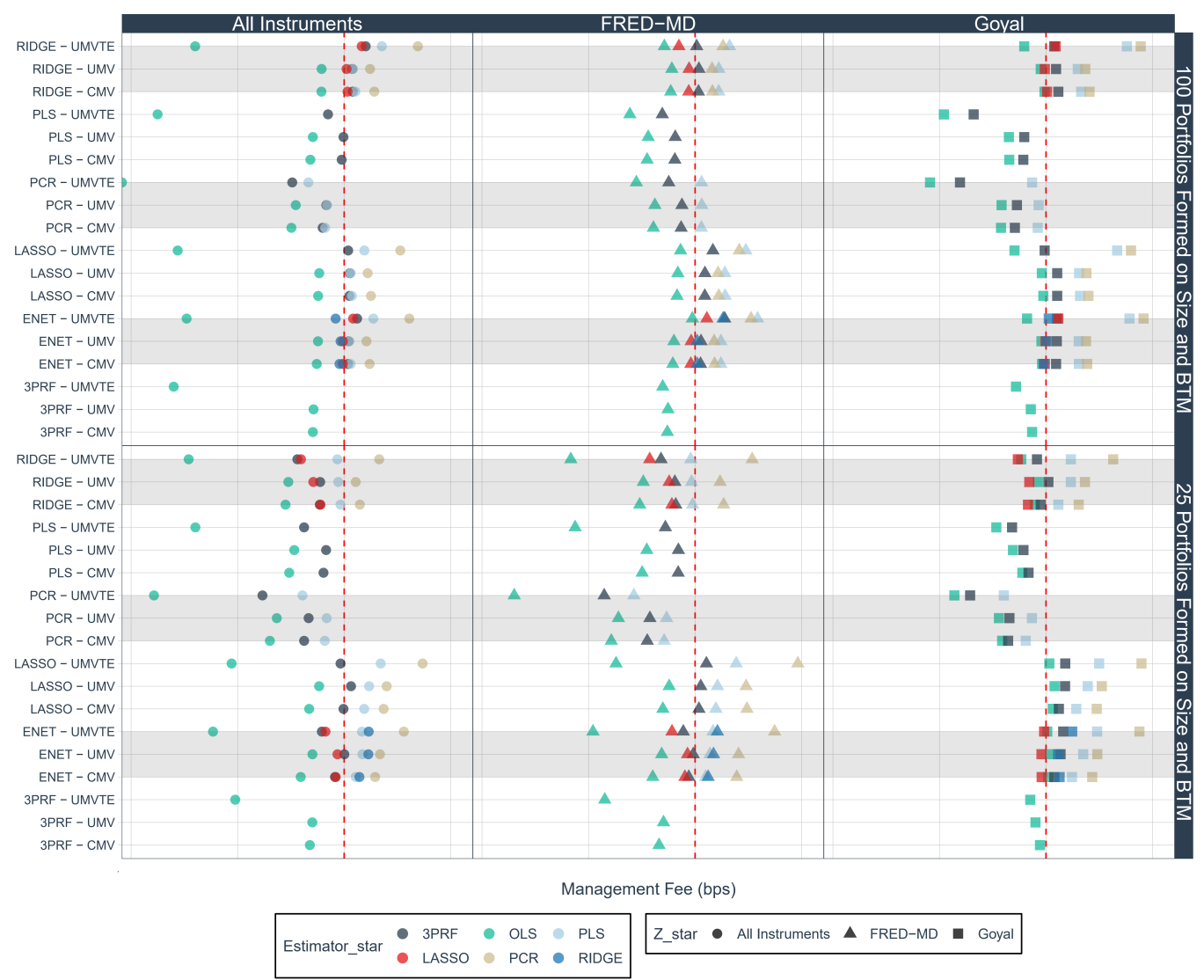

Figure 6 presents the management fee in bps, computed as the solution for $\mathcal{F}$ in equation (36), from an investor switching from an optimal portfolio formed by the estimator and mean-variance framework given in the left axis to another portfolio plotted in different colors and shape. The comparison is done in pairs of optimal portfolios generated by each mean-variance strategy (CMV, UMV, and MVATE), estimators (OLS, 3PRF, PLS, PCR, LASSO, Ridge, and ENet) and sets of conditioning information.

regressions for the remaining sets of conditioning information, as well as additional results. 


\section{Figure 7: $p$-values}
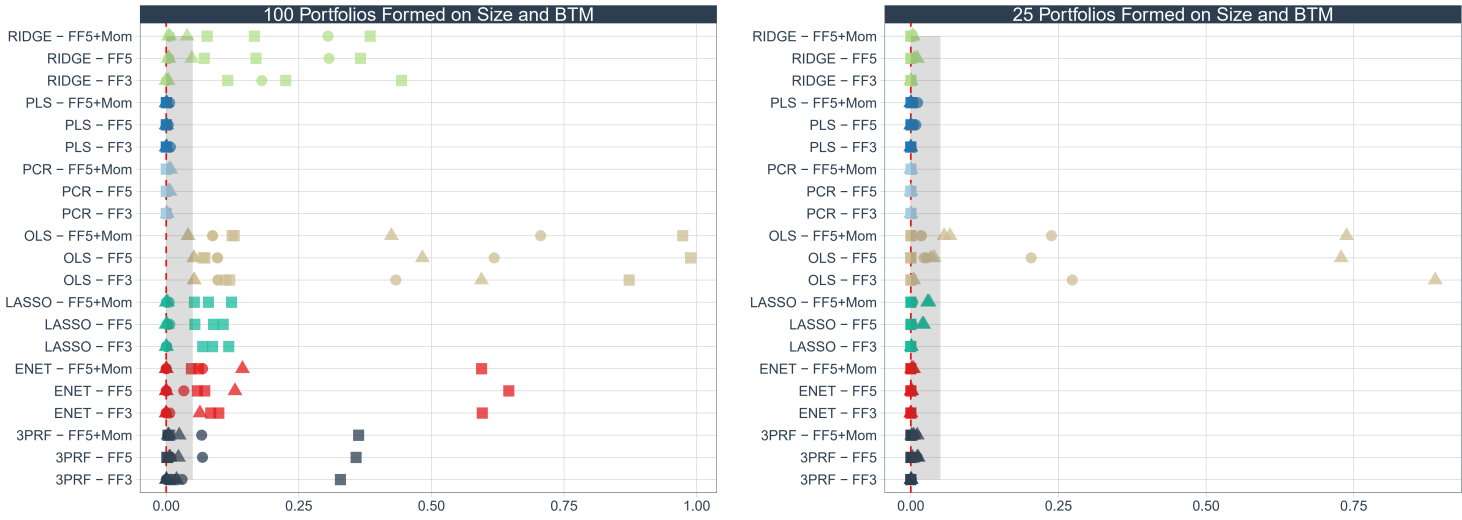

p-value

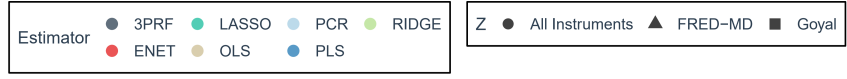

Figure 7 reports the $p$-values of alphas of the regressions of the optimal portfolios generated by each mean-variance strategy (CMV, UMV, and MVATE), estimators (OLS, 3PRF, PLS, PCR, LASSO, Ridge, and ENet) and set of conditioning information used on the Fama-French 3, 5 , and $5+$ momentum factor models. The $p$-values are calculated from Newey-West $t$-statistics computed with one lag.

Figure 8: $R$-Squared
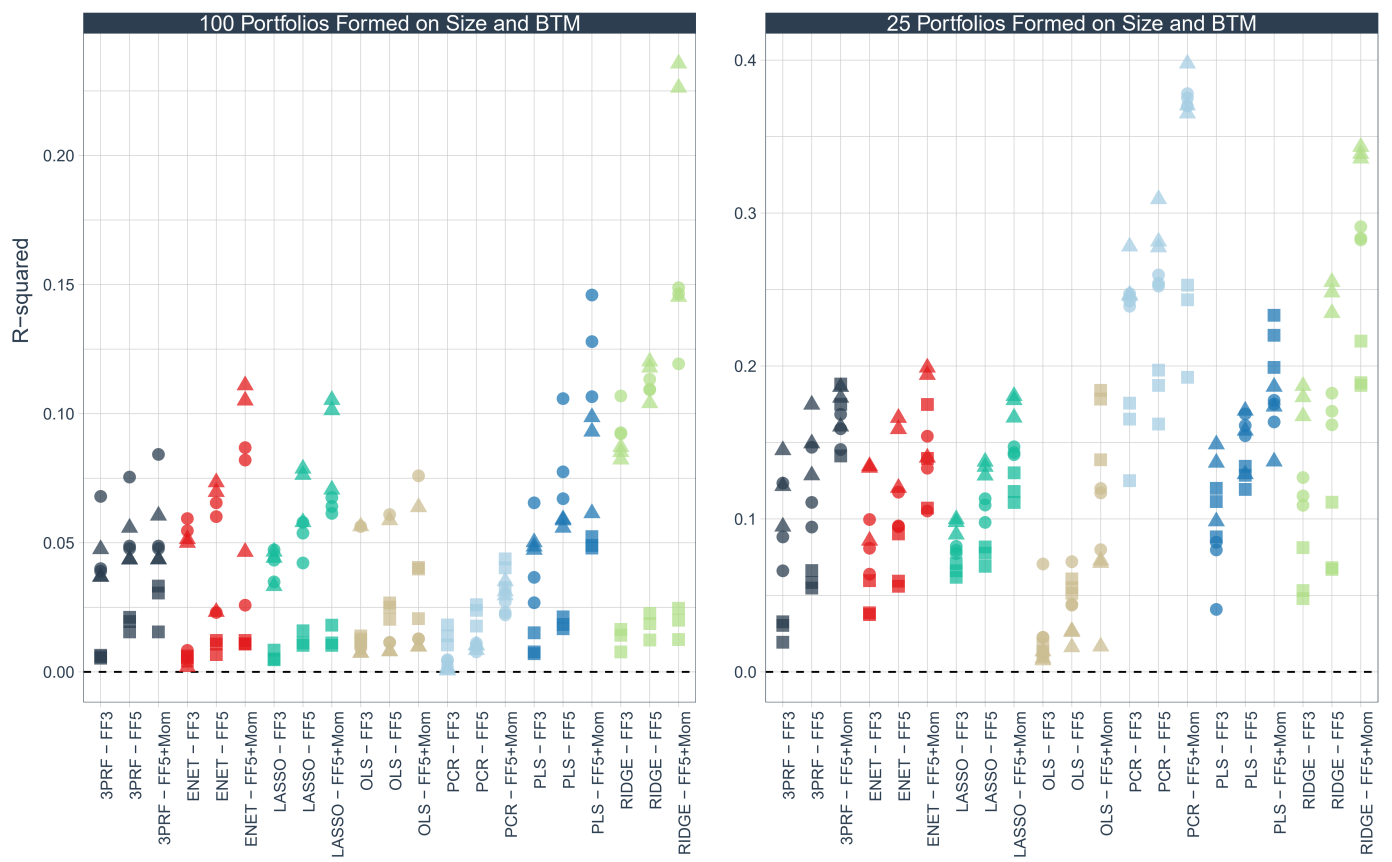

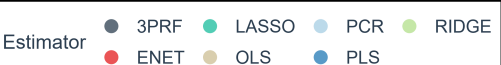

$Z \bullet$ All Instruments $\Delta$ FRED-MD $\boldsymbol{~ G o y a l ~}$

Figure 8 reports the $R^{2}$ of the regressions of the optimal portfolios generated by each mean-variance strategy (CMV, UMV, and MVATE), estimators (OLS, 3PRF, PLS, PCR, LASSO, Ridge, and ENet) and set of conditioning information used on the Fama-French 3, 5, and $5+$ momentum factor models. 
Table 7: Alphas (Monthly \%)

\begin{tabular}{|c|c|c|c|c|c|c|c|c|c|c|c|c|c|c|c|c|c|c|c|}
\hline & & \multicolumn{9}{|c|}{25 Portfolios Formed on Size and Book-to-Market } & \multicolumn{9}{|c|}{100 Portfolios Formed on Size and Book-to-Market } \\
\hline & & \multicolumn{3}{|c|}{ CMV } & \multicolumn{3}{|c|}{ UMV } & \multicolumn{3}{|c|}{ MVATE } & \multicolumn{3}{|c|}{ CMV } & \multicolumn{3}{|c|}{ UMV } & \multicolumn{3}{|c|}{ MVATE } \\
\hline & & FF3 & FF5 & $\begin{array}{l}\text { FF5 + } \\
\text { Mom }\end{array}$ & FF3 & FF5 & $\begin{array}{l}\text { FF5 + } \\
\text { Mom }\end{array}$ & FF3 & FF5 & $\begin{array}{l}\text { FF5 + } \\
\text { Mom }\end{array}$ & FF3 & FF5 & $\begin{array}{l}\text { FF5 + } \\
\text { Mom }\end{array}$ & FF3 & FF5 & $\begin{array}{l}\text { FF5 + } \\
\text { Mom }\end{array}$ & FF3 & FF5 & $\begin{array}{l}\text { FF5 + } \\
\text { Mom }\end{array}$ \\
\hline \multicolumn{20}{|c|}{ Z: FRED-MD } \\
\hline \multirow[t]{4}{*}{ OLS } & $\alpha(\%)$ & 0.027 & 0.021 & 0.019 & 0.027 & 0.021 & 0.019 & -0.037 & -0.082 & -0.077 & 0.017 & 0.016 & 0.016 & 0.017 & 0.016 & 0.016 & 0.188 & 0.219 & 0.248 \\
\hline & $t$-statistics & {$[2.83]$} & {$[2.07]$} & {$[1.84]$} & {$[2.9]$} & {$[2.13]$} & {$[1.92]$} & {$[-0.14]$} & {$[-0.35]$} & {$[-0.34]$} & [1.95] & [1.95] & {$[2.05]$} & {$[1.94]$} & [1.95] & {$[2.05]$} & {$[0.53]$} & {$[0.7]$} & {$[0.8]$} \\
\hline & $p$-val & $0.005^{* *}$ & $0.04^{*}$ & 0.066 & $0.004^{* *}$ & $0.034^{*}$ & 0.056 & 0.888 & 0.729 & 0.738 & 0.052 & 0.052 & $0.041^{*}$ & 0.053 & 0.053 & $0.042^{*}$ & 0.594 & 0.483 & 0.425 \\
\hline & $R^{2}$ & 0.01 & 0.03 & 0.07 & 0.01 & 0.03 & 0.07 & 0.01 & 0.02 & 0.02 & 0.01 & 0.01 & 0.01 & 0.01 & 0.01 & 0.01 & 0.06 & 0.06 & 0.06 \\
\hline \multirow[t]{4}{*}{ 3PRF } & $\alpha(\%)$ & 0.172 & 0.140 & 0.133 & 0.156 & 0.122 & 0.115 & 0.321 & 0.248 & 0.238 & 0.142 & 0.130 & 0.129 & 0.139 & 0.127 & 0.126 & 0.264 & 0.223 & 0.233 \\
\hline & $t$-statistics & {$[3.81]$} & {$[3.04]$} & {$[3.2]$} & [3.54] & {$[2.57]$} & {$[2.84]$} & {$[3.4]$} & {$[2.52]$} & {$[2.56]$} & [3.65] & {$[2.8]$} & {$[2.94]$} & {$[3.49]$} & {$[2.69]$} & {$[2.83]$} & {$[2.35]$} & {$[2.29]$} & {$[2.26]$} \\
\hline & $p$-val & $0^{* *}$ & $0.003^{* *}$ & $0.002^{* *}$ & $0^{* *}$ & $0.011^{*}$ & $0.005^{* *}$ & $0.001^{* *}$ & $0.012^{*}$ & $0.011^{*}$ & $0^{* *}$ & $0.006^{* *}$ & $0.004^{* *}$ & $0.001^{* *}$ & $0.008^{* *}$ & $0.005^{* *}$ & $0.02^{*}$ & $0.023^{*}$ & $0.025^{*}$ \\
\hline & $R^{2}$ & 0.12 & 0.15 & 0.18 & 0.09 & 0.13 & 0.16 & 0.14 & 0.17 & 0.19 & 0.04 & 0.04 & 0.04 & 0.04 & 0.04 & 0.04 & 0.05 & 0.06 & 0.06 \\
\hline \multirow[t]{4}{*}{ PLS } & $\alpha(\%)$ & 0.246 & 0.215 & 0.208 & 0.224 & 0.188 & 0.181 & 0.479 & 0.404 & 0.394 & 0.240 & 0.245 & 0.236 & 0.235 & 0.238 & 0.230 & 0.461 & 0.507 & 0.501 \\
\hline & $t$-statistics & [4.61] & {$[4.08]$} & {$[4.03]$} & {$[4.28]$} & {$[4.12]$} & {$[4.05]$} & [4.08] & [3.77] & [3.64] & [4.58] & {$[4.06]$} & {$[4.4]$} & {$[4.4]$} & [4.14] & [4.38] & {$[3.27]$} & {$[3.41]$} & {$[3.33]$} \\
\hline & $p$-val & $0^{* *}$ & $0^{* *}$ & $0^{* *}$ & $0^{* *}$ & $0^{* *}$ & $0^{* *}$ & $0^{* *}$ & $0^{* *}$ & $0^{* *}$ & $0^{* *}$ & $0^{* *}$ & $0^{* *}$ & $0^{* *}$ & $0^{* *}$ & $0^{* *}$ & $0.001^{* *}$ & $0.001^{* *}$ & $0.001 * *$ \\
\hline & $R^{2}$ & 0.14 & 0.16 & 0.17 & 0.15 & 0.17 & 0.19 & 0.10 & 0.13 & 0.14 & 0.05 & 0.06 & 0.10 & 0.05 & 0.06 & 0.09 & 0.05 & 0.06 & 0.06 \\
\hline \multirow[t]{4}{*}{ PCR } & $\alpha(\%)$ & 0.379 & 0.321 & 0.300 & 0.346 & 0.291 & 0.271 & 0.742 & 0.622 & 0.582 & 0.217 & 0.205 & 0.195 & 0.211 & 0.196 & 0.187 & 0.427 & 0.397 & 0.379 \\
\hline & $t$-statistics & {$[6.19]$} & {$[5.47]$} & {$[5.32]$} & {$[5.99]$} & {$[5.02]$} & {$[4.99]$} & {$[6.09]$} & {$[5.53]$} & {$[5.19]$} & {$[3.1]$} & {$[2.84]$} & {$[2.79]$} & {$[3.23]$} & {$[2.9]$} & {$[2.77]$} & {$[3.07]$} & {$[2.69]$} & {$[2.64]$} \\
\hline & $p$-val & $0^{* *}$ & $0^{* *}$ & $0^{* *}$ & $0^{* *}$ & $0^{* *}$ & $0^{* *}$ & $0^{* *}$ & $0^{* *}$ & $0^{* *}$ & $0.002^{* *}$ & $0.005^{* *}$ & $0.006^{* *}$ & $0.001^{* *}$ & $0.004^{* *}$ & $0.006^{* *}$ & $0.002^{* *}$ & $0.008^{* *}$ & $0.009^{* *}$ \\
\hline & $R^{2}$ & 0.25 & 0.28 & 0.37 & 0.28 & 0.31 & 0.40 & 0.25 & 0.28 & 0.37 & 0.00 & 0.01 & 0.03 & 0.00 & 0.01 & 0.04 & 0.00 & 0.01 & 0.03 \\
\hline \multirow[t]{4}{*}{ LASSO } & $\alpha(\%)$ & 0.157 & 0.114 & 0.103 & 0.138 & 0.101 & 0.092 & 0.296 & 0.213 & 0.193 & 0.099 & 0.084 & 0.081 & 0.096 & 0.080 & 0.077 & 0.190 & 0.155 & 0.149 \\
\hline & $t$-statistics & {$[3.35]$} & {$[2.35]$} & {$[2.21]$} & {$[3.39]$} & {$[2.31]$} & {$[2.19]$} & {$[3.34]$} & {$[2.32]$} & {$[2.17]$} & {$[4.87]$} & {$[4.2]$} & [3.99] & {$[4.75]$} & [4] & {$[3.8]$} & {$[3.55]$} & {$[3.11]$} & {$[3.01]$} \\
\hline & $p$-val & $0.001^{* *}$ & $0.019^{*}$ & $0.028^{*}$ & $0.001^{* *}$ & $0.022^{*}$ & $0.029^{*}$ & $0.001^{* *}$ & $0.021^{*}$ & $0.031^{*}$ & $0^{* *}$ & $0^{* *}$ & $0^{* *}$ & $0^{* *}$ & $0^{* *}$ & $0^{* *}$ & $0^{* * *}$ & $0.002^{* * *}$ & $0.003^{* *}$ \\
\hline & $R^{2}$ & 0.10 & 0.13 & 0.18 & 0.09 & 0.13 & 0.17 & 0.10 & 0.14 & 0.18 & 0.04 & 0.08 & 0.10 & 0.05 & 0.08 & 0.11 & 0.03 & 0.06 & 0.07 \\
\hline \multirow[t]{4}{*}{ RIDGE } & $\alpha(\%)$ & 0.247 & 0.171 & 0.150 & 0.238 & 0.169 & 0.149 & 0.470 & 0.325 & 0.290 & 0.127 & 0.106 & 0.096 & 0.122 & 0.101 & 0.091 & 0.263 & 0.207 & 0.189 \\
\hline & $t$-statistics & {$[4.19]$} & {$[2.56]$} & {$[3.04]$} & [3.53] & {$[2.56]$} & {$[2.86]$} & {$[4.5]$} & {$[2.75]$} & {$[3.01]$} & [4.32] & {$[2.92]$} & {$[2.9]$} & [4.21] & {$[2.83]$} & {$[2.74]$} & {$[2.91]$} & {$[1.98]$} & {$[2.07]$} \\
\hline & $p$-val & $0^{* *}$ & $0.011^{*}$ & $0.003^{* *}$ & $0^{* *}$ & $0.011^{*}$ & $0.005^{* *}$ & $0^{* *}$ & $0.006^{* *}$ & $0.003^{* *}$ & $0^{* *}$ & $0.004^{* *}$ & $0.004^{* *}$ & $0^{* *}$ & $0.005^{* *}$ & $0.007^{* *}$ & $0.004^{* *}$ & $0.049^{*}$ & $0.039^{*}$ \\
\hline & $R^{2}$ & 0.18 & 0.25 & 0.34 & 0.17 & 0.23 & 0.34 & 0.19 & 0.25 & 0.34 & 0.09 & 0.12 & 0.23 & 0.09 & 0.12 & 0.24 & 0.08 & 0.10 & 0.15 \\
\hline \multirow[t]{4}{*}{ ENET } & $\alpha(\%)$ & 0.195 & 0.159 & 0.149 & 0.163 & 0.128 & 0.121 & 0.395 & 0.315 & 0.302 & 0.117 & 0.102 & 0.098 & 0.112 & 0.097 & 0.093 & 0.151 & 0.117 & 0.106 \\
\hline & $t$-statistics & {$[4.3]$} & {$[3.47]$} & {$[3.34]$} & {$[4.32]$} & {$[3.16]$} & {$[2.84]$} & [4.21] & {$[3.51]$} & {$[3.03]$} & {$[4.63]$} & [4.11] & [3.89] & {$[4.58]$} & [3.93] & {$[3.8]$} & {$[1.86]$} & {$[1.52]$} & {$[1.47]$} \\
\hline & $p$-val & $0^{* *}$ & $0.001^{* *}$ & $0.001^{* *}$ & $0^{* *}$ & $0.002^{* *}$ & $0.005^{* *}$ & $0^{* *}$ & $0.001^{* *}$ & $0.003^{* *}$ & $0^{* *}$ & $0^{* *}$ & $0^{* *}$ & $0^{* *}$ & $0^{* *}$ & $0^{* *}$ & 0.064 & 0.130 & 0.144 \\
\hline & $R^{2}$ & 0.13 & 0.16 & 0.20 & 0.13 & 0.17 & 0.19 & 0.09 & 0.12 & 0.14 & 0.05 & 0.07 & 0.11 & 0.05 & 0.07 & 0.11 & 0.00 & 0.02 & 0.05 \\
\hline
\end{tabular}

Table 7 reports the alphas, $t$-statistics, $p$-values and the $R^{2}$ of the regressions of the optimal portfolios generated by each mean-variance strategy (CMV, UMV, and MVATE), estimators (OLS, 3PRF, PLS, PCR, LASSO, Ridge, and ENet) using the FRED-MD as conditioning information on the Fama-French 3, 5, and $5+$ momentum factor models. FRED-MD is a large dataset containing 128 macroeconomic and financial variables. 


\subsection{Financial Metrics}

As a final analysis, we also assess how sparsity and dimension reduction can affect common financial metrics for the returns generated by the efficient portfolios. We check how the turnover is affected by different estimators. Additionally, we also assess another common risk metric, which is the maximum drawdown. Related to it, we evaluate the pain ratie 28 . As in Kirby and Ostdiek (2012), we define the turnover as

$$
\text { Turnover }_{p}=\frac{1}{T} \sum_{t \in \tau_{O O S}} \sum_{i}^{N}\left|x_{p, i, t}-x_{p, i, t-1}\right|+\left|\sum_{i}^{N}\left(x_{p, i, t}-x_{p, i, t-1}\right)\right|
$$

where $x_{p, i, t}$ is the optimal weight of asset $i$ for a portfolio $p$ at $t$ in the OOS, and $x_{p, i, t-1}$ is the optimal weight of asset $i$ for a portfolio $p$ at $t-1$. Following Gu et al. (2018), we define the maximum drawdown as

$$
\operatorname{MaxDD}_{p}=\max _{0 \leq Y_{p, t_{1} \leq Y_{p, t_{2}} \leq T}}\left(Y_{p, t_{1}}-Y_{p, t_{2}}\right)
$$

where $Y_{p, t}$ is the cumulative log return for a portfolio $p$ from the first OOS period to $t$. Given the large amount of results, we report only for the CMV approach ${ }^{29}$ An inspection in table 8 show that overall all estimator generate high turnover, especially when the available number of risky assets $N$ is low. Notice that PLS, in general, produces a lower rate of turnover compared to the penalized regressors for most of the cases. This behavior, as similarly noted by Gu et al. (2018), should be understood in light of the large role of price trend predictors selected by these dimension reduction techniques. The maximum drawdown for PLS and PCR are in line with the other estimators. It is clear that, with more risky assets, the diversification generates a lower drawdown. We see that the pain ratio also shows that, in general, for large $N$, PCR and PLS performs well compared to most estimators.

\section{Conclusion}

The financial literature has collected a large list of variables that potentially could be in investors' conditioning information sets. Standard approaches relying on a few lagged

${ }^{28}$ The Pain Ratio can be calculated as

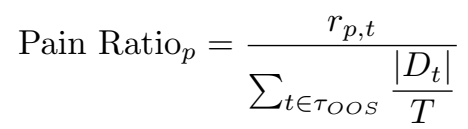

where $D_{\tau}$ is the drawdown since previous peak.

${ }^{29}$ For the other two approaches, UMV and MVATE, the results are similar. 
Table 8: Turnover and Financial Metrics (Monthly \%)

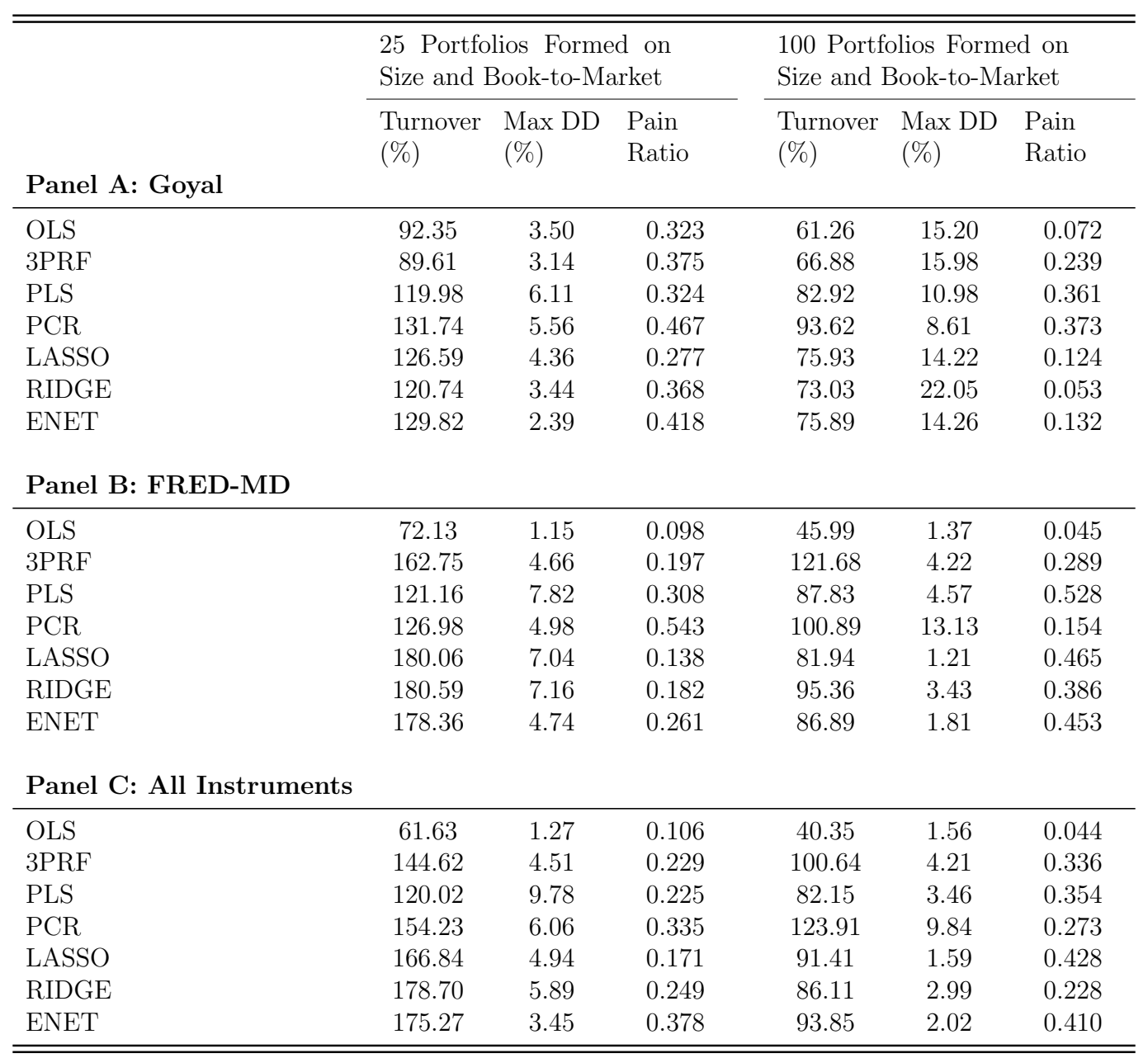

Table 8 reports several standard financial metrics to evaluate portfolios by estimator for the CMV framework for both portfolios. The Turnover is computed following equation (38). MaxDD is maximum drawdown as presented in equation (39). Pain Ratio is the standard metric, as shown in equation (37). Panel A reports the financial metrics generated when the variables from Goyal's website are used as $\mathbf{Z}$. Goyal variables comprises: $b / m, d f r, d f y$, infl, ltr, lty, ntis, svar, tms and tbl. Panel B presents the financial metrics obtained using the FRED-MD variables. Finally, panel $\mathrm{C}$ shows the financial metrics when all variables are used as conditioning information. "All Instruments" is the combination of Goyal and FRED-MD datasets with the Economic Policy Uncertainty (EPU) index and the Financial Stress Indicator (FSI). 
variables and in a naive OLS estimation can be problematic and inefficient, as shown in this work.

We exploit the wealth set of data as predictive signals and condense this information when estimating the conditional mean using high-dimensional data. We do this using many different techniques in order to impose sparsity and dimensionality reduction when finding the conditional mean, which is the most important item driving the formation of mean-variance efficient portfolios with conditioning information. We evaluate how penalized estimators, such as LASSO, Ridge and Elastic Net, as well as pure dimensionality reduction and latent factors approaches as Partial Least Squares (PLS) and Principal Components Regression (PCR), in addition to a generalization of the former (Three Pass Regression Filter) can produce different optimized portfolios.

We assess these methods in a high-dimensional setting for the predictive list of instruments. We see that it is possible to build efficient portfolios, for all three different approaches evaluated, delivering on average higher Sharpe ratios OOS, implied Sharpe ratios $\left(S R^{*}\right)$, and higher certainty equivalent returns (CER). We find that PLS and PCR can enhance the formation of optimized portfolios, generating large and significant alphas when evaluated in factor models, such as Fama-French 3 and 5.

In short, exploiting a high-dimensional set of conditioning information, it is possible with a "bet on sparsity", employing shrinkage-type estimators, regularization, and allowing flexible forms for the approximation of the conditional mean of the excess returns. 


\section{References}

Abhyankar, A., Basu, D., and Stremme, A. (2012). The optimal use of return predictability: An empirical study. Journal of Financial and Quantitative Analysis, 47(5):973-1001.

Bai, J. and Ng, S. (2006). Confidence intervals for diffusion index forecasts and inference for factor-augmented regressions. Econometrica, 74(4):1133-1150.

Baker, S. R., Bloom, N., and Davis, S. J. (2016). Measuring economic policy uncertainty. The quarterly journal of economics, 131(4):1593-1636.

Bergmeir, C., Hyndman, R. J., and Koo, B. (2018). A note on the validity of cross-validation for evaluating autoregressive time series prediction. Computational Statistics 83 Data Analysis, 120:70-83.

Bianchi, D., Büchner, M., and Tamoni, A. (2019). Bond risk premia with machine learning. USC-INET Research Paper, (19-11).

Brandt, M. W. (2010). Portfolio choice problems. In Handbook of financial econometrics: Tools and techniques, pages 269-336. Elsevier.

Brandt, M. W. and Santa-Clara, P. (2006). Dynamic portfolio selection by augmenting the asset space. The Journal of Finance, 61(5):2187-2217.

Campbell, J. Y. and Thompson, S. B. (2007). Predicting excess stock returns out of sample: Can anything beat the historical average? The Review of Financial Studies, 21(4):15091531.

Chiang, I.-H. E. (2015). Modern portfolio management with conditioning information. Journal of Empirical Finance, 33:114-134.

Chinco, A., Clark-Joseph, A. D., and Ye, M. (2019). Sparse signals in the cross-section of returns. The Journal of Finance, 74(1):449-492.

Cochrane, J. H. (2009). Asset Pricing. Princeton University Press.

Cochrane, J. H. (2011). Presidential address: Discount rates. The Journal of finance, 66(4):1047-1108.

Diebold, F. X., Ghysels, E., Mykland, P., and Zhang, L. (2019). Big data in dynamic predictive econometric modeling. 
Feng, G., Giglio, S., and Xiu, D. (2017). Taming the factor zoo. Fama-Miller Working Paper, 24070.

Ferson, W. E. and Siegel, A. F. (2001). The efficient use of conditioning information in portfolios. The Journal of Finance, 56(3):967-982.

Ferson, W. E. and Siegel, A. F. (2009). Testing portfolio efficiency with conditioning information. Review of Financial Studies, 22(7):2735-2758.

Fleming, J., Kirby, C., and Ostdiek, B. (2001). The economic value of volatility timing. The Journal of Finance, 56(1):329-352.

Fletcher, J. and Basu, D. (2016). An examination of the benefits of dynamic trading strategies in uk closed-end funds. International Review of Financial Analysis, 47:109-118.

Frank, L. E. and Friedman, J. H. (1993). A statistical view of some chemometrics regression tools. Technometrics, 35(2):109-135.

Freyberger, J., Neuhierl, A., and Weber, M. (2017). Dissecting characteristics nonparametrically. Technical report, National Bureau of Economic Research.

Friedman, J., Hastie, T., and Tibshirani, R. (2001). The elements of statistical learning, volume 1. Springer series in statistics New York.

Goto, S. and $\mathrm{Xu}, \mathrm{Y}$. (2015). Improving mean variance optimization through sparse hedging restrictions. Journal of Financial and Quantitative Analysis, 50(6):1415-1441.

Green, J., Hand, J. R., and Zhang, X. F. (2013). The supraview of return predictive signals. Review of Accounting Studies, 18(3):692-730.

Gu, S., Kelly, B., and Xiu, D. (2018). Empirical asset pricing via machine learning. Technical report, National Bureau of Economic Research.

Hansen, L. P. and Richard, S. F. (1987). The role of conditioning information in deducing testable restrictions implied by dynamic asset pricing models. Econometrica, pages 587613.

Harvey, C. R., Liu, Y., and Zhu, H. (2016). ... and the cross-section of expected returns. The Review of Financial Studies, 29(1):5-68.

Hastie, T., Tibshirani, R., and Wainwright, M. (2015). Statistical learning with sparsity: the lasso and generalizations. Chapman and Hall/CRC. 
Huang, J.-z. and Shi, Z. (2011). Determinants of bond risk premia. Technical report, Citeseer.

Jurado, K., Ludvigson, S. C., and Ng, S. (2015). Measuring uncertainty. American Economic Review, 105(3):1177-1216.

Kelly, B. and Pruitt, S. (2013). Market expectations in the cross-section of present values. The Journal of Finance, 68(5):1721-1756.

Kelly, B. and Pruitt, S. (2015). The three-pass regression filter: A new approach to forecasting using many predictors. Journal of Econometrics, 186(2):294-316.

Kelly, B. T., Pruitt, S., and Su, Y. (2019). Characteristics are covariances: A unified model of risk and return. Journal of Financial Economics.

Kirby, C. and Ostdiek, B. (2012). It's all in the timing: simple active portfolio strategies that outperform naive diversification. Journal of Financial and Quantitative Analysis, 47(2):437-467.

Kozak, S., Nagel, S., and Santosh, S. (2019). Shrinking the cross-section. Journal of Financial Economics.

Ledoit, O. and Wolf, M. (2008). Robust performance hypothesis testing with the sharpe ratio. Journal of Empirical Finance, 15(5):850-859.

Light, N., Maslov, D., and Rytchkov, O. (2017). Aggregation of information about the cross section of stock returns: A latent variable approach. The Review of Financial Studies, 30(4):1339-1381.

McCracken, M. W. and Ng, S. (2016). Fred-md: A monthly database for macroeconomic research. Journal of Business \&3 Economic Statistics, 34(4):574-589.

McLean, R. D. and Pontiff, J. (2016). Does academic research destroy stock return predictability? The Journal of Finance, 71(1):5-32.

Peñaranda, F. (2016). Understanding portfolio efficiency with conditioning information. Journal of Financial and Quantitative Analysis, 51(3):985-1011.

Politis, D. N. and Romano, J. P. (1992). A circular block-resampling procedure for stationary data. Exploring the limits of bootstrap, 2635270.

Püttmann, L. (2018). Patterns of panic: Financial crisis language in historical newspapers. 
Stock, J. H. and Watson, M. W. (1996). Evidence on structural instability in macroeconomic time series relations. Journal of Business 85 Economic Statistics, 14(1):11-30.

Stock, J. H. and Watson, M. W. (2002a). Forecasting using principal components from a large number of predictors. Journal of the American statistical association, 97(460):1167-1179.

Stock, J. H. and Watson, M. W. (2002b). Macroeconomic forecasting using diffusion indexes. Journal of Business \& Economic Statistics, 20(2):147-162.

Stone, M. and Brooks, R. J. (1990). Continuum regression: cross-validated sequentially constructed prediction embracing ordinary least squares, partial least squares and principal components regression. Journal of the Royal Statistical Society: Series B (Methodological), $52(2): 237-258$.

Tibshirani, R. (1996). Regression shrinkage and selection via the lasso. Journal of the Royal Statistical Society: Series B (Methodological), 58(1):267-288.

Welch, I. and Goyal, A. (2007). A comprehensive look at the empirical performance of equity premium prediction. The Review of Financial Studies, 21(4):1455-1508.

Zou, H. and Hastie, T. (2005). Regularization and variable selection via the elastic net. Journal of the royal statistical society: series B (statistical methodology), 67(2):301-320. 


\section{Internet Appendix}

\section{A Appendix - Statistical Inference for the Difference of Sharpe Ratios}

To test for the difference of the Sharpe ratios of two different optimal portfolios, we follow Ledoit and Wolf (2008) approach to perform these tests. The main advantage of this method, is its robustness to (i) when returns have tails heavier than the normal distribution, and (ii) serial correlation of the actual returns or of the squared returns (volatility clustering). Following Ledoit and Wolf (2008) the difference between $S R_{a}$ and $S R_{b}$, for two optimal portfolios produced by two different estimators, a and b, is given by

$$
\Delta=S R^{(a)}-S R^{(b)}=\frac{\mu^{(a)}}{\sigma^{(a)}}-\frac{\mu^{(b)}}{\sigma^{(b)}}
$$

where $\hat{\mu}$ and $\hat{\sigma}$ are the OOS unconditional mean and variance of the excess returns from the optimal portfolio generated by an estimator. Thus, the null hypothesis can be written as: $H_{0}: \Delta=0$. Denote the vector of uncentered population and sample moments of the excess returns generated by two different estimators by

$$
\begin{aligned}
& \zeta=\left[\mu^{(a)}, \mu^{(b)}, \mathbb{E}\left(r^{2^{(a)}}\right), \mathbb{E}\left(r^{2^{(b)}}\right)\right]^{\top} \\
& \widehat{\zeta}=\left[\widehat{\mu^{(a)}}, \widehat{\mu^{(b)}}, \widehat{\left.\mathbb{E}\left(r^{2^{(a)}}\right), \widehat{\mathbb{E}\left(r^{2^{(b)}}\right)}\right]^{\top}}\right.
\end{aligned}
$$

Under the assumption that the differences of these moments converges in distribution to a Normal distribution with mean 0 and variance $\Psi$, using the delta method we have

$$
\sqrt{T}(\widehat{\Delta}-\Delta) \stackrel{d}{\rightarrow} N\left(0, \nabla^{\top} f(\zeta) \Psi \nabla f(\zeta)\right)
$$

where $f(\zeta)=\frac{\mu^{(a)}}{\sqrt{\mathbb{E}\left(r^{2^{(a)}}\right)-\mu^{2^{(a)}}}}-\frac{\mu^{(b)}}{\sqrt{\mathbb{E}\left(r^{2^{(b)}}\right)-\mu^{2^{(b)}}}}$ and the standard error of $\widehat{\Delta}$ is given by

$$
\operatorname{se}(\widehat{\Delta})=\sqrt{\frac{\nabla^{\top} f(\widehat{\zeta}) \widehat{\Psi} \nabla f(\widehat{\zeta})}{T}}
$$

Since neither the returns, nor the squared returns of financial assets are generally an i.i.d. process, we need to use a robust estimator. One evident way to obtain $\widehat{\Psi}$ is using HAC kernel estimation. Another way is to use bootstrap inference. Ledoit and Wolf (2008) suggest to 
construct a studentized time series bootstrap confidence interval for the difference of the SRs. This method has been shown to be robust when returns have tails heavier than the normal distribution or are of time series nature. The bootstrap data is generated using the circular block bootstrap of Politis and Romano (1992). The two-sided distribution function of the studentized statistic can be obtained via bootstrap as follows:

$$
f\left(\frac{|\widehat{\Delta}-\Delta|}{s e(\widehat{\Delta})}\right) \approx f\left(\frac{\left|\widehat{\Delta}^{\text {boot }}-\Delta\right|}{s e\left(\widehat{\Delta}^{\text {boot }}\right)}\right)
$$

where $f(\cdot)$ is the distribution of a random variable, $\Delta$ is populational difference between $S R_{a}$ and $S R_{b}, \widehat{\Delta}$ is the sample counterpart of this difference obtained in the data in the estimation window, and $\widehat{\Delta}^{\text {boot }}$ is the estimated difference computed from bootstrap. The standard errors are denoted by $s e(\cdot)$. Out of the distribution obtained from the bootstrap in the equation (44), we can find the confidence interval for $\Delta$ and the p-values of the test ${ }^{30}$.

\footnotetext{
${ }^{30}$ For further details in this procedure, see Ledoit and Wolf (2008).
} 


\section{B Appendix - Additional Results}

B.1 Out-of-Sample Analysis - Sharpe Ratios

B.1.1 Performance Evaluation 
Table B.1: Out-of-Sample Sharpe ratios delivered by each estimator and set of conditioning information

\begin{tabular}{|c|c|c|c|c|c|c|c|c|c|c|c|c|}
\hline \multirow[b]{3}{*}{ Estimator } & \multicolumn{6}{|c|}{5 Industry Portfolios } & \multicolumn{6}{|c|}{6 Portfolios Formed on Size and Book-to-Market } \\
\hline & \multicolumn{2}{|c|}{ CMV } & \multicolumn{2}{|c|}{ UMV } & \multicolumn{2}{|c|}{ MVATE } & \multicolumn{2}{|c|}{ CMV } & \multicolumn{2}{|c|}{ UMV } & \multicolumn{2}{|c|}{ MVATE } \\
\hline & $S R$ & $p$-val & $S R$ & $p$-val & $S R$ & $p$-val & $S R$ & $p$-val & $S R$ & $p$-val & $S R$ & $p$-val \\
\hline \multicolumn{13}{|l|}{ Panel A: Goyal } \\
\hline OLS & 0.133 & 0.032 & 0.100 & 0.107 & 0.135 & 0.030 & 0.207 & 0.001 & 0.218 & 0.001 & 0.203 & 0.001 \\
\hline $3 \mathrm{PRF}$ & 0.125 & 0.044 & 0.083 & 0.181 & 0.125 & 0.043 & 0.222 & 0.000 & 0.258 & 0.000 & 0.223 & 0.000 \\
\hline PLS & 0.121 & 0.051 & 0.109 & 0.078 & 0.119 & 0.054 & 0.247 & 0.000 & 0.246 & 0.000 & 0.247 & 0.000 \\
\hline PCR & 0.089 & 0.149 & 0.076 & 0.217 & 0.085 & 0.168 & 0.298 & 0.000 & 0.299 & 0.000 & 0.300 & 0.000 \\
\hline LASSO & 0.209 & 0.001 & 0.141 & 0.024 & 0.209 & 0.001 & 0.168 & 0.007 & 0.187 & 0.003 & 0.166 & 0.008 \\
\hline RIDGE & 0.131 & 0.035 & 0.112 & 0.072 & 0.131 & 0.034 & 0.173 & 0.006 & 0.224 & 0.000 & 0.174 & 0.005 \\
\hline ENET & 0.163 & 0.009 & 0.130 & 0.037 & 0.168 & 0.007 & 0.231 & 0.000 & 0.260 & 0.000 & 0.236 & 0.000 \\
\hline \multicolumn{13}{|c|}{ Panel B: FRED-MD } \\
\hline OLS & 0.005 & 0.934 & 0.009 & 0.881 & -0.046 & 0.455 & 0.154 & 0.013 & 0.158 & 0.011 & 0.037 & 0.550 \\
\hline $3 \mathrm{PRF}$ & 0.072 & 0.246 & 0.009 & 0.883 & 0.073 & 0.239 & 0.186 & 0.003 & 0.197 & 0.002 & 0.188 & 0.003 \\
\hline PLS & 0.102 & 0.099 & 0.046 & 0.455 & 0.101 & 0.102 & 0.210 & 0.001 & 0.198 & 0.002 & 0.201 & 0.001 \\
\hline PCR & 0.115 & 0.064 & 0.019 & 0.764 & 0.112 & 0.070 & 0.272 & 0.000 & 0.255 & 0.000 & 0.275 & 0.000 \\
\hline LASSO & 0.156 & 0.012 & 0.056 & 0.363 & 0.152 & 0.014 & 0.226 & 0.000 & 0.201 & 0.001 & 0.226 & 0.000 \\
\hline RIDGE & 0.116 & 0.061 & 0.028 & 0.655 & 0.116 & 0.062 & 0.242 & 0.000 & 0.230 & 0.000 & 0.246 & 0.000 \\
\hline ENET & 0.127 & 0.042 & 0.032 & 0.601 & 0.125 & 0.044 & 0.261 & 0.000 & 0.261 & 0.000 & 0.261 & 0.000 \\
\hline \multicolumn{13}{|c|}{ Panel C: All Instruments } \\
\hline OLS & -0.035 & 0.594 & -0.002 & 0.979 & -0.078 & 0.240 & 0.160 & 0.017 & 0.168 & 0.012 & -0.002 & 0.973 \\
\hline $3 \mathrm{PRF}$ & 0.067 & 0.281 & 0.029 & 0.640 & 0.068 & 0.271 & 0.193 & 0.002 & 0.213 & 0.001 & 0.191 & 0.002 \\
\hline PLS & 0.002 & 0.976 & 0.037 & 0.581 & -0.001 & 0.991 & 0.264 & 0.000 & 0.263 & 0.000 & 0.265 & 0.000 \\
\hline PCR & 0.133 & 0.047 & 0.040 & 0.552 & 0.129 & 0.054 & 0.238 & 0.000 & 0.237 & 0.001 & 0.240 & 0.000 \\
\hline LASSO & 0.122 & 0.067 & 0.052 & 0.435 & 0.118 & 0.078 & 0.271 & 0.000 & 0.228 & 0.000 & 0.274 & 0.000 \\
\hline RIDGE & 0.113 & 0.091 & 0.035 & 0.602 & 0.112 & 0.094 & 0.248 & 0.000 & 0.229 & 0.001 & 0.247 & 0.000 \\
\hline ENET & 0.069 & 0.301 & 0.047 & 0.483 & 0.069 & 0.300 & 0.255 & 0.000 & 0.234 & 0.000 & 0.266 & 0.000 \\
\hline
\end{tabular}

Table B.1 summarises the OOS (Jan-1996 to Dec-2017) Sharpe ratios (SR) by estimator and optimal portfolio framework (CMV, UMV and MVATE) for the 5 industry portfolios and 6 portfolios formed on Size/BTM. Panel A reports the Sharpe ratios generated when the variables from Goyal's website are used as Z. Goyal variables comprises: b/m, dfr, dfy, infl, ltr, lty, ntis, svar, tms and tbl. Panel B presents the Sharpe ratios obtained using the FRED-MD variables. The FRED-MD is a large dataset containing 128 macroeconomic and financial variables. Finally, panel C shows the Sharpe rations when all variables are used as conditioning information. "All Instruments" is the combination of Goyal and FRED-MD datasets with the Economic Policy Uncertainty (EPU) index and the Financial Stress Indicator (FSI). The $p$-val is the p-value from the two-sided test of the SR. 


\section{B.1.2 Statistical Inference for the Difference of Sharpe Ratios}

Table B.2: Test for the difference of the Sharpe ratios - OOS (Jan-1996 - Dec-2017) - 6 Portfolios Formed on Size and Book-to-Market
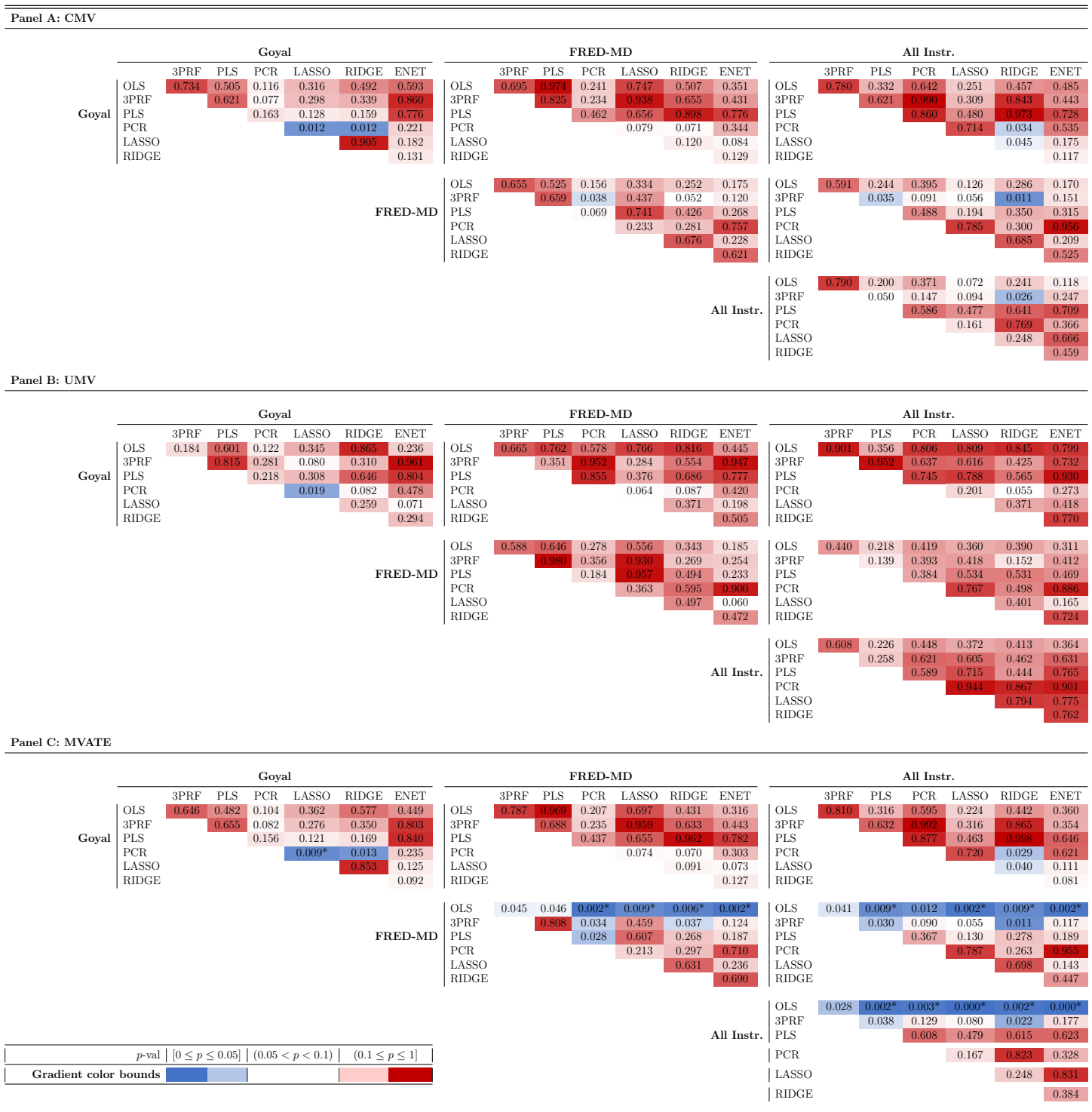

Test for the differences of the Sharpe ratios of the OOS (Jan-1996 - Dec-2017) returns of the efficient portfolios formed from the dataset with 6 Size/BTM portfolios using 7 different estimators (OLS, 3PRF, PLS, PCR, LASSO, RIDGE and ENet) and three different set of conditioning information (Goyal, FRED-MD and "All Instruments"). Each panel shows the test of pairs of Sharpe ratios for three different framework to build efficient portfolios. Panel A reports conditionally mean-variance (CMV) efficient portfolios. Panel B reports unconditionally mean-variance efficient portfolios. Panel $\mathrm{C}$ presents the mean-variance tracking error (MVATE) portfolios. We split the results depending on conditioning information set used. Goyal variables comprises: $b / m, d f r$, dfy, infl, ltr, lty, ntis, svar, tms and $t b l$. The FRED-MD is a large dataset containing 128 macroeconomic and financial variables. Finally, "All Instruments" is the combination of Goyal and FRED-MD datasets with the Economic Policy Uncertainty (EPU) index and the Financial Stress Indicator (FSI). 
Table B.3: Test for the difference of the Sharpe ratios - OOS (Jan-1996 - Dec-2017) - 5 Portfolios Formed on Size and Book-to-Market

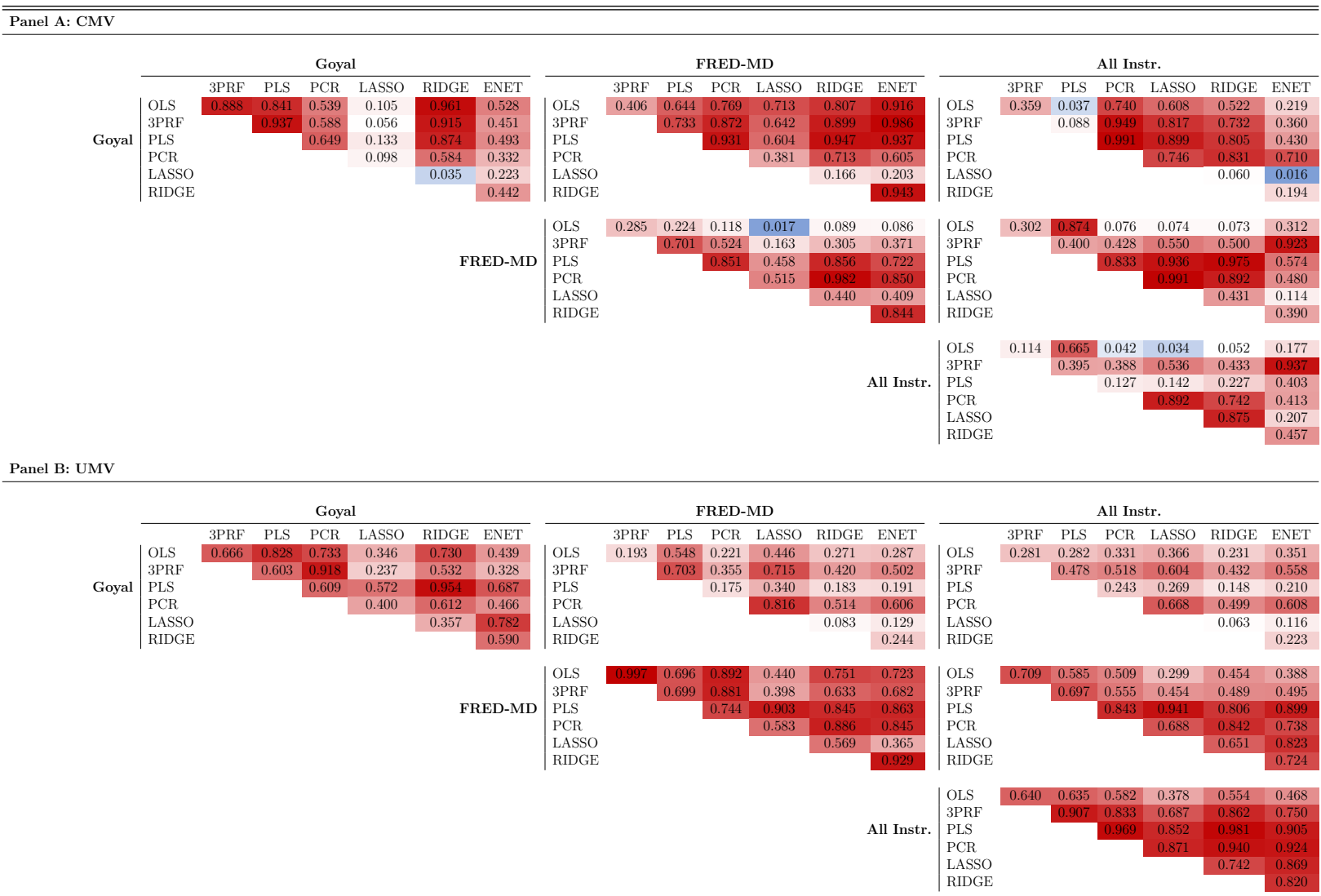

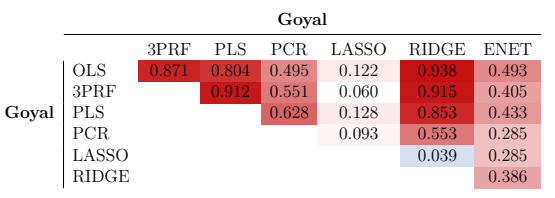

FRED-MD

\begin{tabular}{r|c|c|}
$p$-val $|[0 \leq p \leq 0.05]|(0.05<p<0.1)$ & $(0.1 \leq p \leq 1]$ \\
\hline Gradient color bounds &
\end{tabular}

3PRF
PLS
PCR
LASS
RIDGE

RIDGE

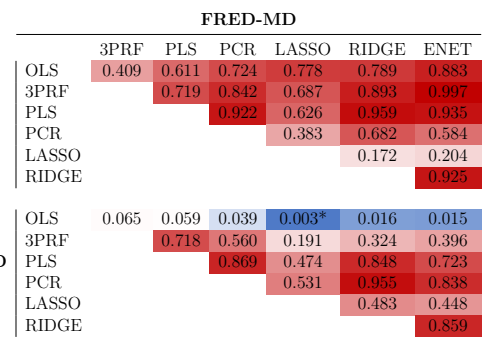

All Inst

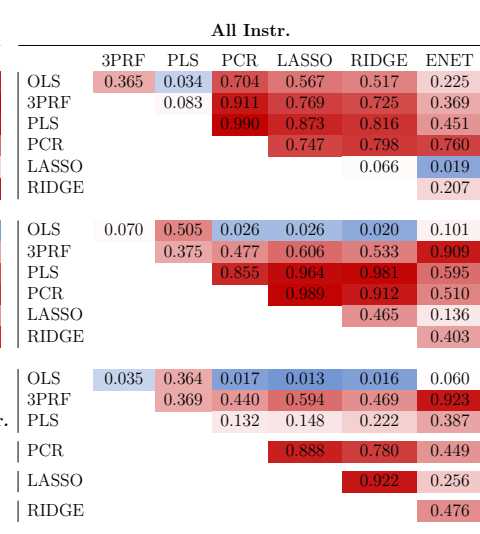

Test for the differences of the Sharpe ratios of the OOS (jan-1996 - Dec-2017) returns of the efficient portfolios formed from the dataset with 5 industry portfolios using 7 different estimators (OLS, 3PRF, PLS, PCR, LASSO, RIDGE and ENet) and three different set of conditioning information (Goyal, FRED-MD and "All Instruments"). Each panel shows the test of pairs of Sharpe ratios for three different framework to build efficient portfolios. Panel A reports conditionally mean-variance (CMV) efficient portfolios. Panel B reports unconditionally mean-variance efficient portfolios. Panel C presents the mean-variance tracking error (MVATE) portfolios. We split the results depending on conditioning information set used. Goyal variables comprises: $b / m$, dfr, dfy, infl, ltr, lty, ntis, svar, tms and tbl. The FRED-MD is a large dataset containing 128 macroeconomic and financial variables. Finally, "All Instruments" is the combination of Goyal and FRED-MD datasets with the Economic Policy Uncertainty (EPU) index and the Financial Stress Indicator (FSI). 


\section{B.2 Characteristics and the Distribution of Optimized Portfolio Weights}

Figure B.1: Distribution of Optimized Portfolio Weights - 6 Portfolios Formed on Size and Book-to-Market

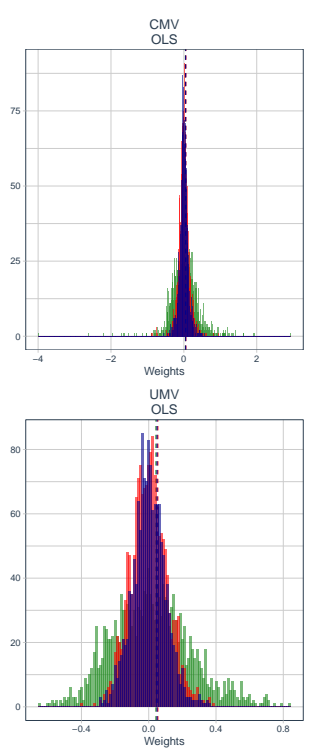

UMUTE

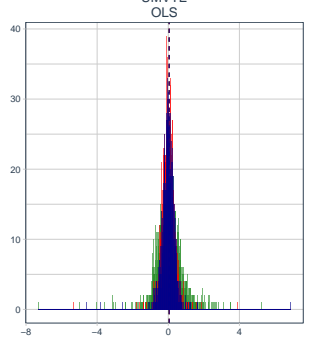

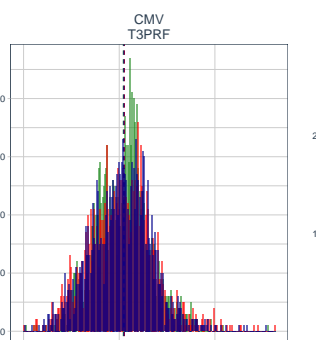

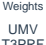

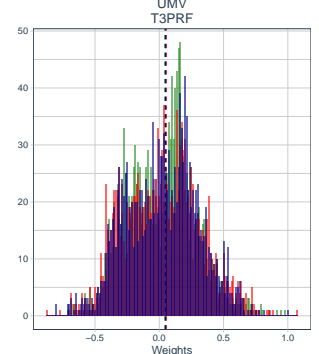

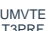

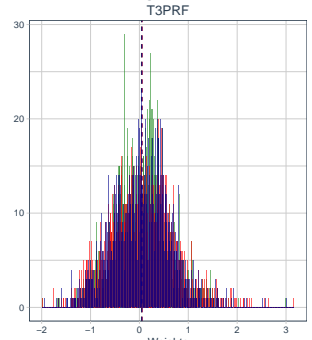

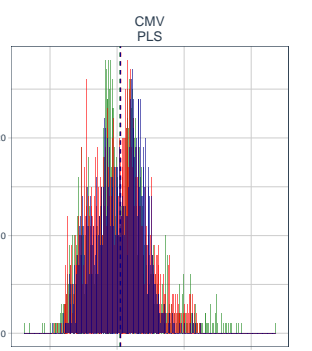

oun

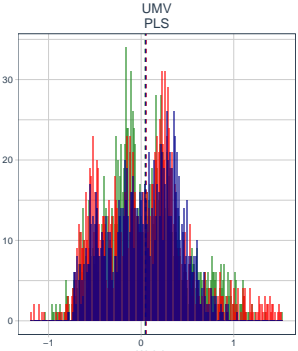

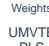

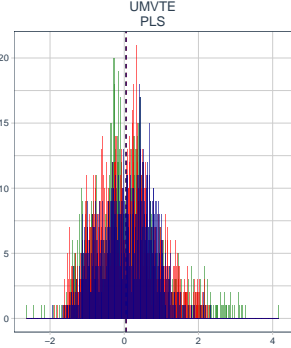

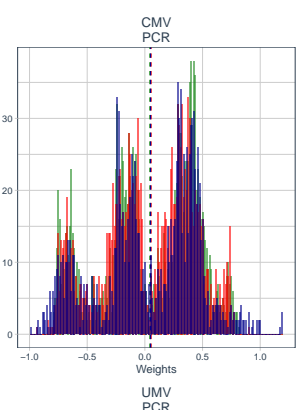

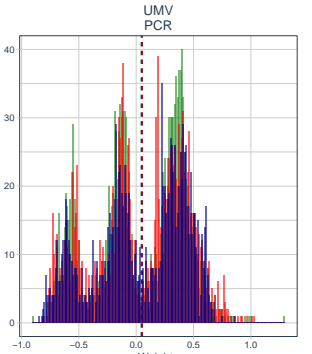

UnuTre

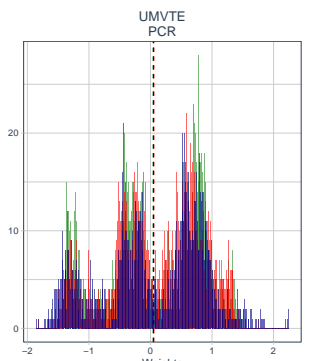

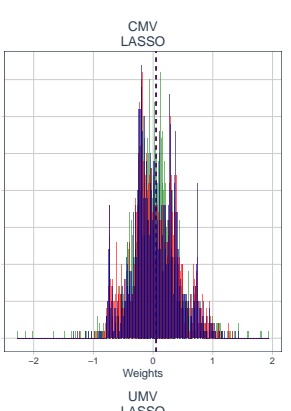

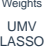

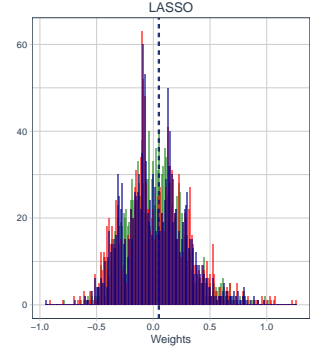

unwre

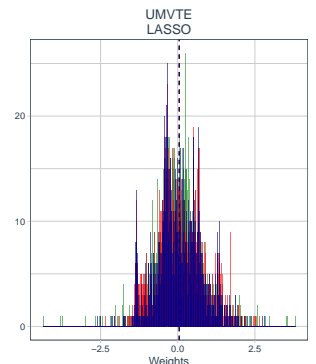

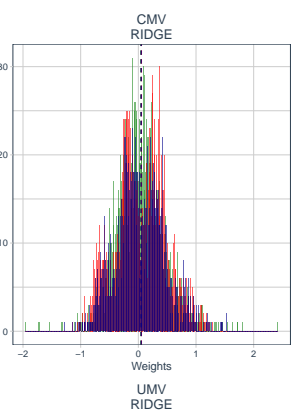

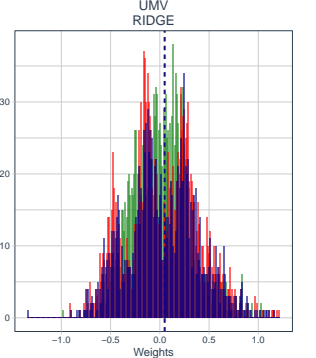

unvre

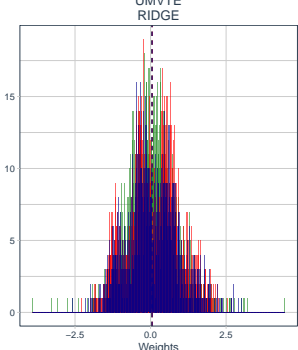

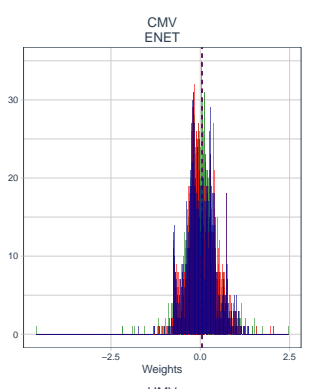

unv

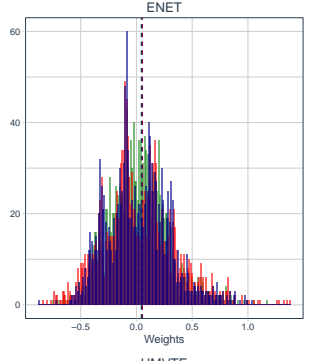

WNTET

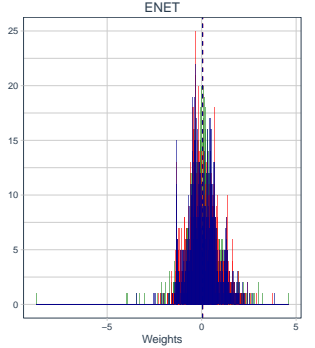

Figure B.1 plots the distribution of the optimized portfolios weights generated by each estimator (columns) and mean-variance optimal framework (rows) for the 6 portfolios formed on Size/BTM. The first row reports the CMV strategies,, the second row reports the UMV, and the third one plots the UMVTE. The set of conditioning information used in $\mathbf{Z}$ are plotted in different colors: (i) Goyal's in green, (ii) FRED-MD in red, and (iii) "All Instruments" in blue. Goyal's variables comprises: $b / m$, dfr, dfy, infl, ltr, lty, ntis, svar, tms and tbl. FRED-MD is a large dataset containing 128 macroeconomic and financial variables. "All Instruments" is the combination of Goyal and FRED-MD datasets with the Economic Policy Uncertainty (EPU) index and the Financial Stress Indicator (FSI). 
Figure B.2: Distribution of Optimized Portfolio Weights - 5 Industry Portfolios

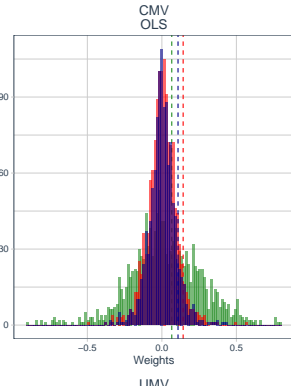

UMY

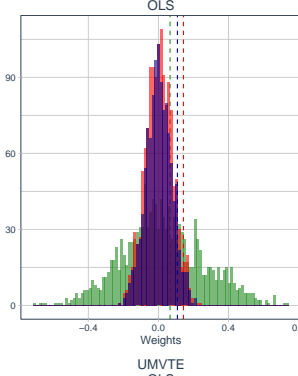

oLs
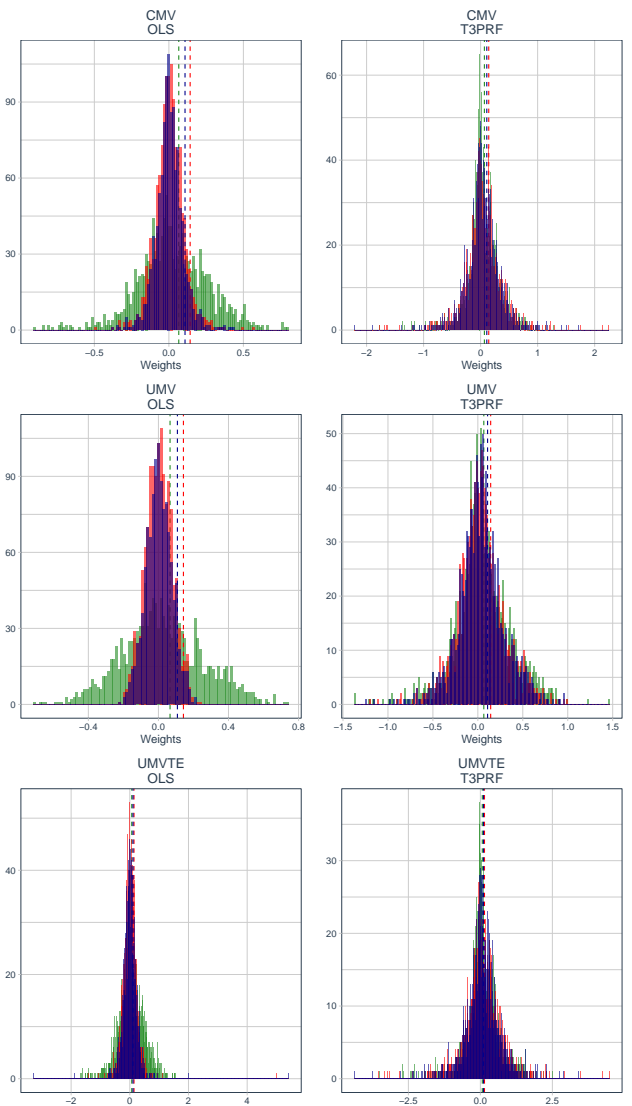

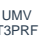

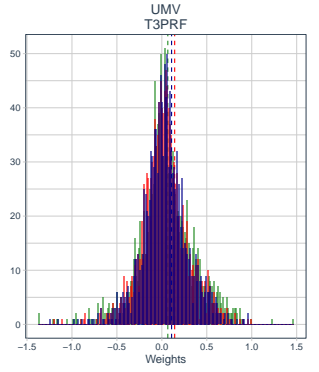

UMVTE
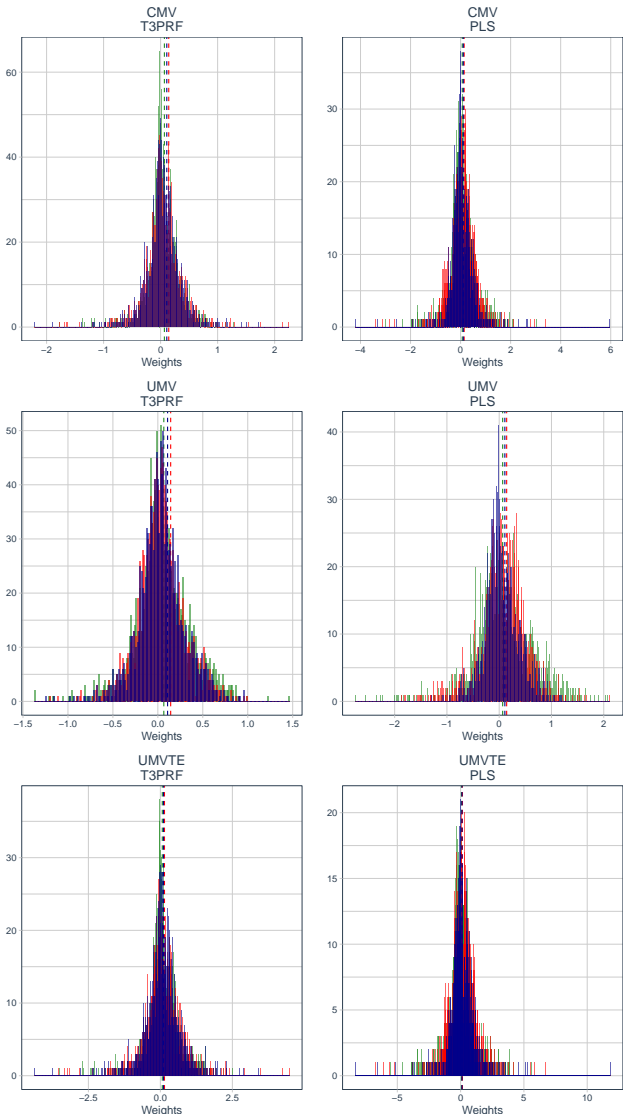

UMVTE

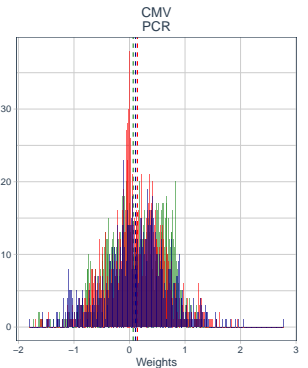

WMights
PCR

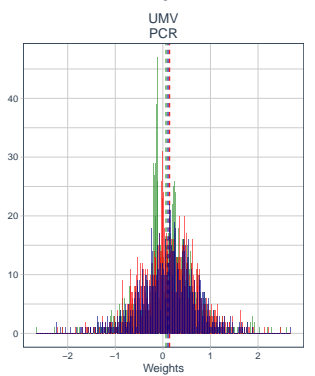

Weights
UMVTE
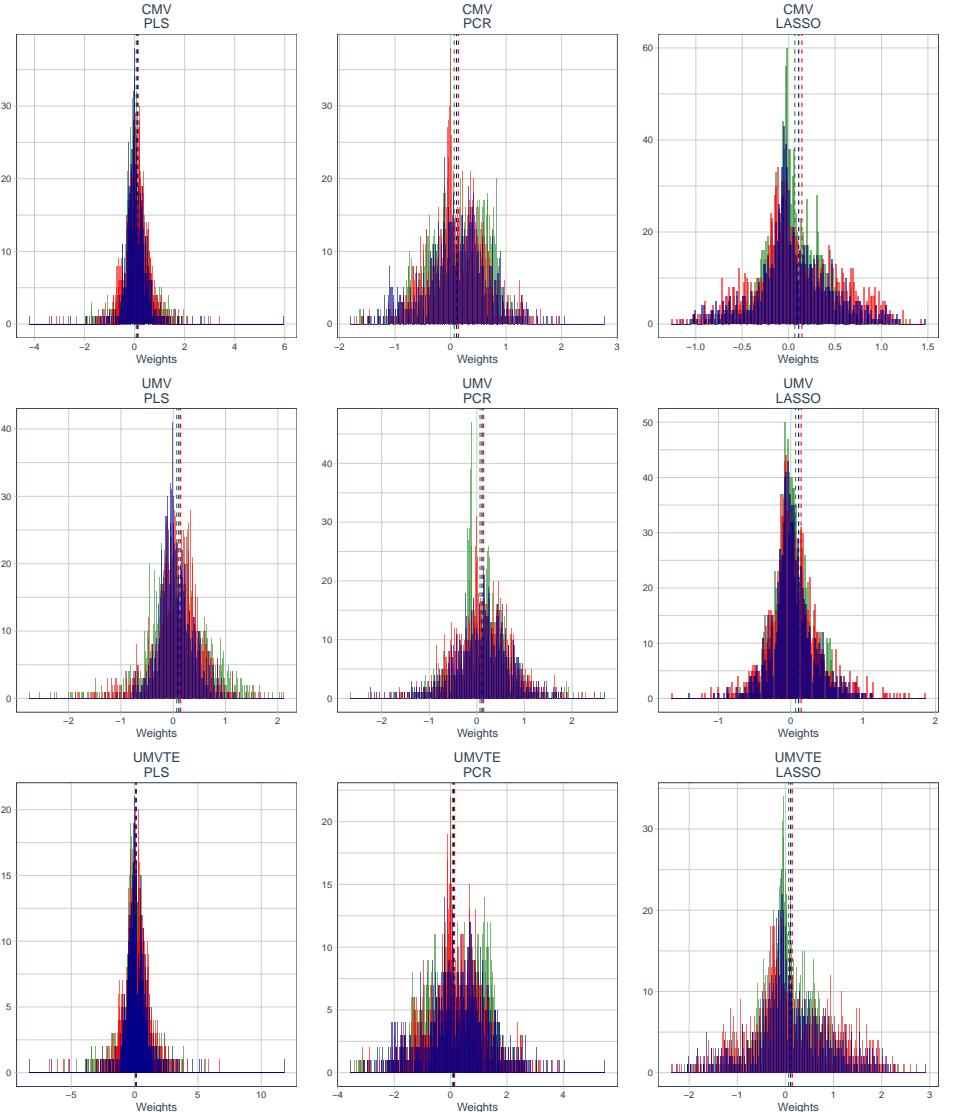

\begin{tabular}{l}
0.0 \\
Weights \\
UMV \\
\hline UASSO
\end{tabular}

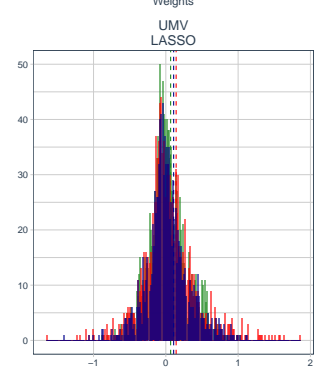

Wuights
UMVTE
LASSO

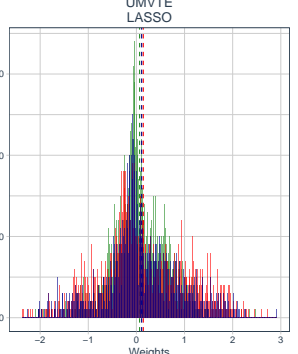

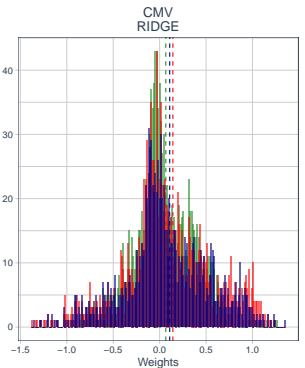

Weights
UMV
RIDGE

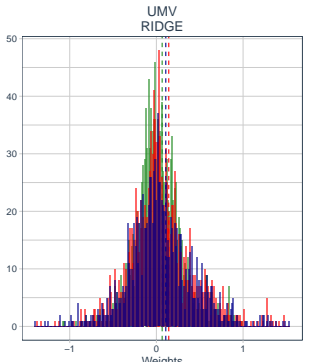

Weights
UMVTE
UNTEE

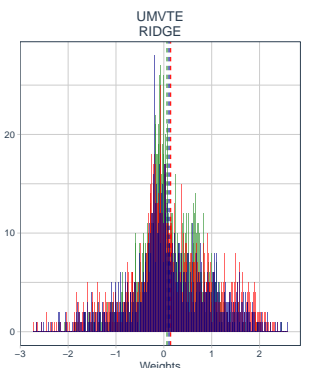

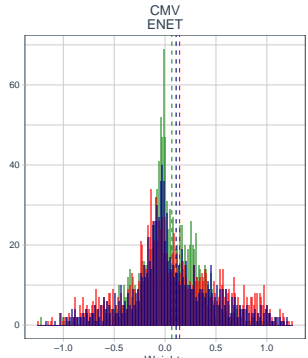

UMights
ENET

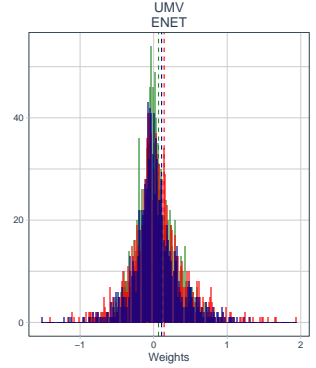

UMVTE

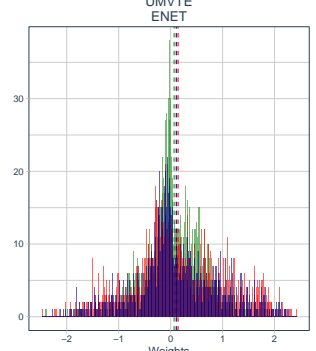

Figure B.2 plots the distribution of the optimized portfolios weights generated by each estimator (columns) and mean-variance optimal framework (rows) for the 5 industry portfolios. The first row reports the CMV strategies,, the second row reports the UMV, and the third one plots the UMVTE. The set of conditioning information used in $\mathbf{Z}$ are plotted in different colors: (i) Goyal's in green, (ii) FRED-MD in red, and (iii) "All Instruments" in blue. Goyal's variables comprises: $b / m, d f r, d f y$, infl, ltr, lty, ntis, svar, tms and tbl. FRED-MD is a large dataset containing 128 macroeconomic and financial variables. "All Instruments" is the combination of Goyal and FRED-MD datasets with the Economic Policy Uncertainty (EPU) index and the Financial Stress Indicator (FSI). 


\section{B.3 Variable Contribution}

Figure B.3: Variable Contribution by Estimator and Set of Conditioning Information - 6 Portfolios Formed on Size and Book-to-Market
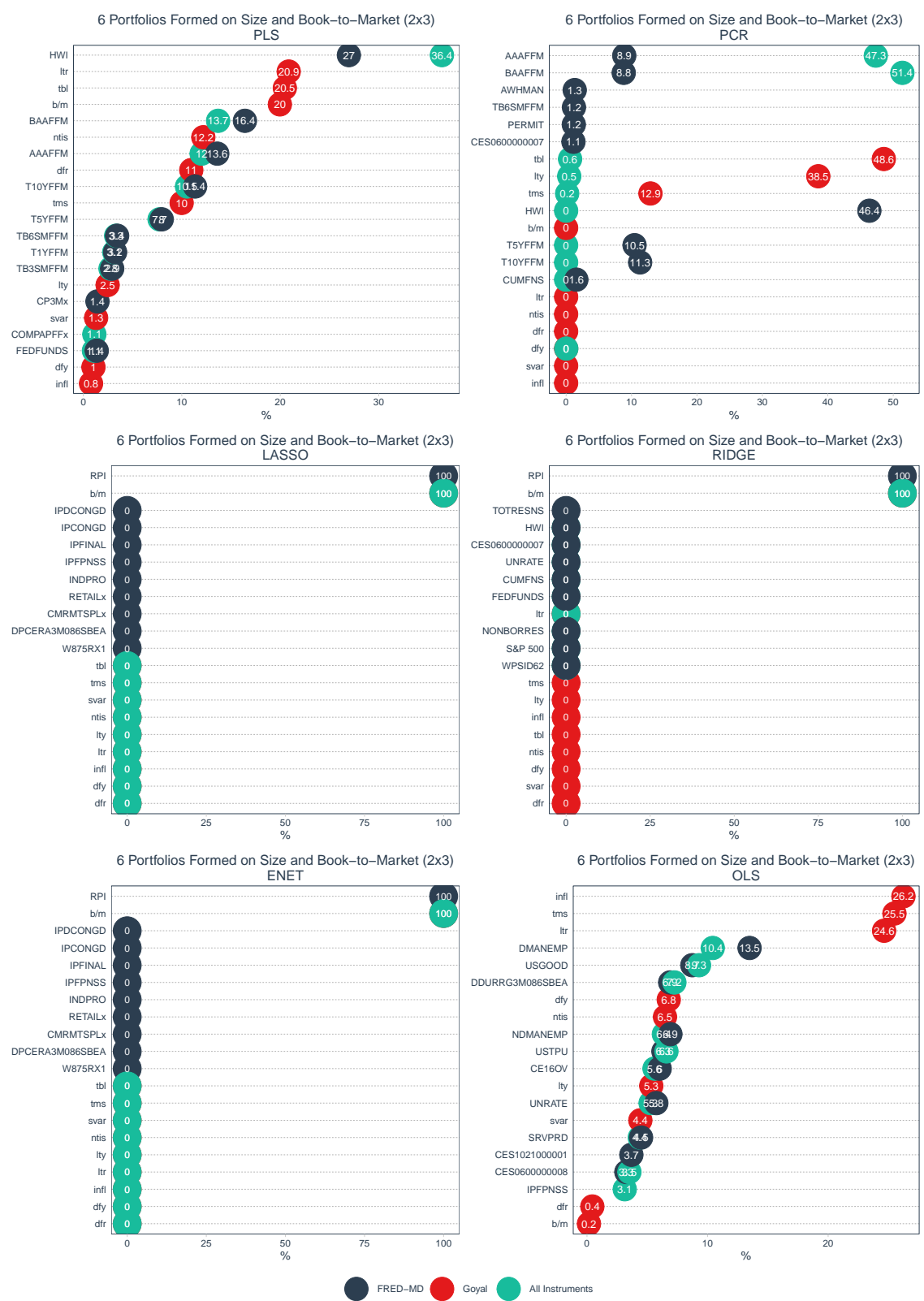

Figure B.3 reports the 10 most influential variables by estimator (panels) and set of conditioning information used (colors) for the for the 6 portfolios formed on Size/BTM. We take a direct approach to obtain the contribution for each variable. At each month $t$, we compute the absolute values of the estimated coefficients in (6) for each asset $i$ and lagged variable $k$ in the conditioning set. We destandardize the variables whenever necessary. Grouping by estimators and $\mathbf{Z}$ used, we pool all the estimates of the $N$ assets and calculate the average of the absolute values. In order to make the comparison clear, we normalize these means to sum one. 
Figure B.4: Variable Contribution by Estimator and Set of Conditioning Information - 5 Industry Portfolios
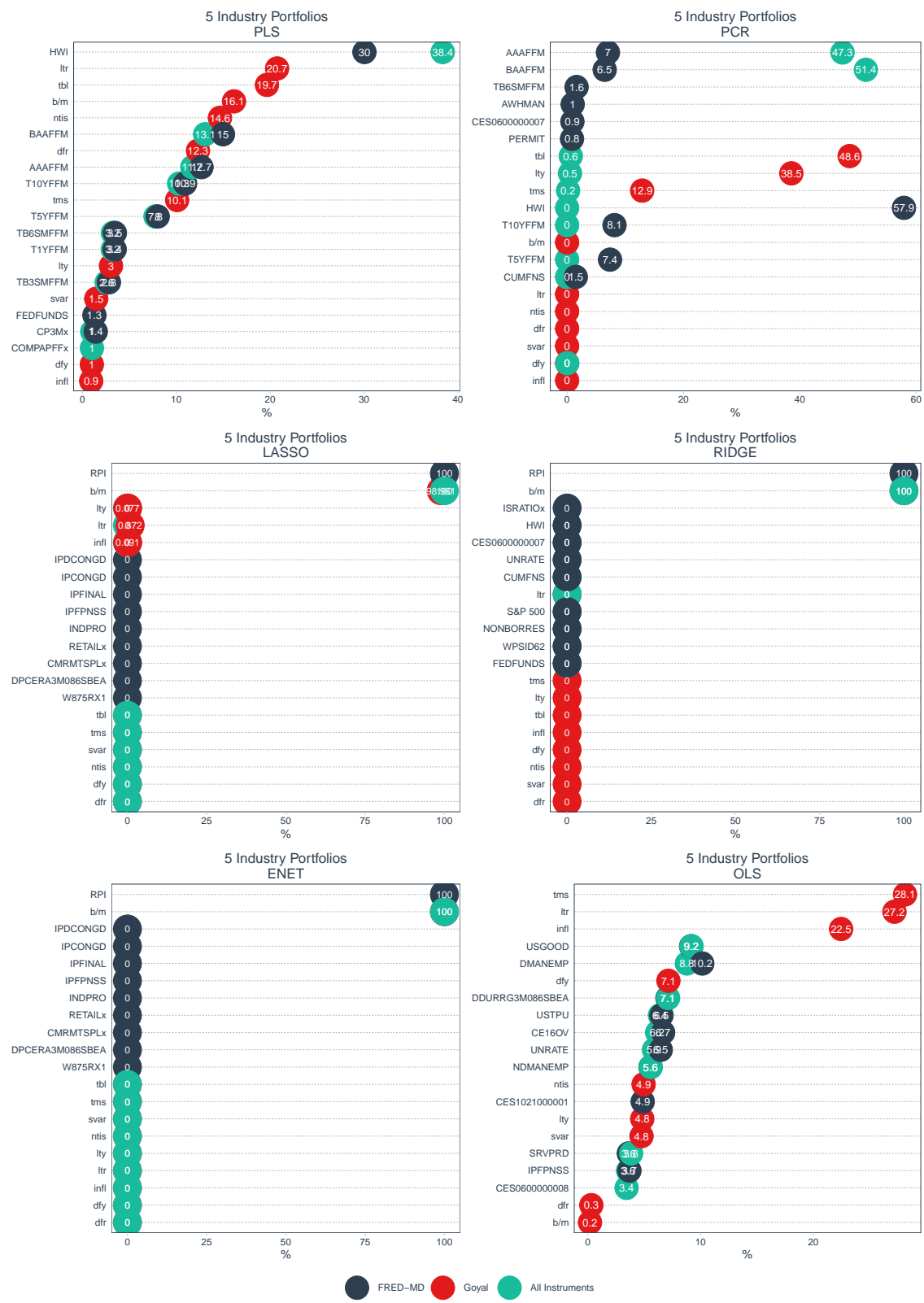

Figure B.4 reports the 10 most influential variables by estimator (panels) and set of conditioning information used (colors) for the for the 5 industry portfolios. We take a direct approach to obtain the contribution for each variable. At each month $t$, we compute the absolute values of the estimated coefficients in (6) for each asset $i$ and lagged variable $k$ in the conditioning set. We destandardize the variables whenever necessary. Grouping by estimators and $\mathbf{Z}$ used, we pool all the estimates of the $N$ assets and calculate the average of the absolute values. In order to make the comparison clear, we normalize these means to sum one. 
Figure B.5: Out-of-Sample $R^{2}$
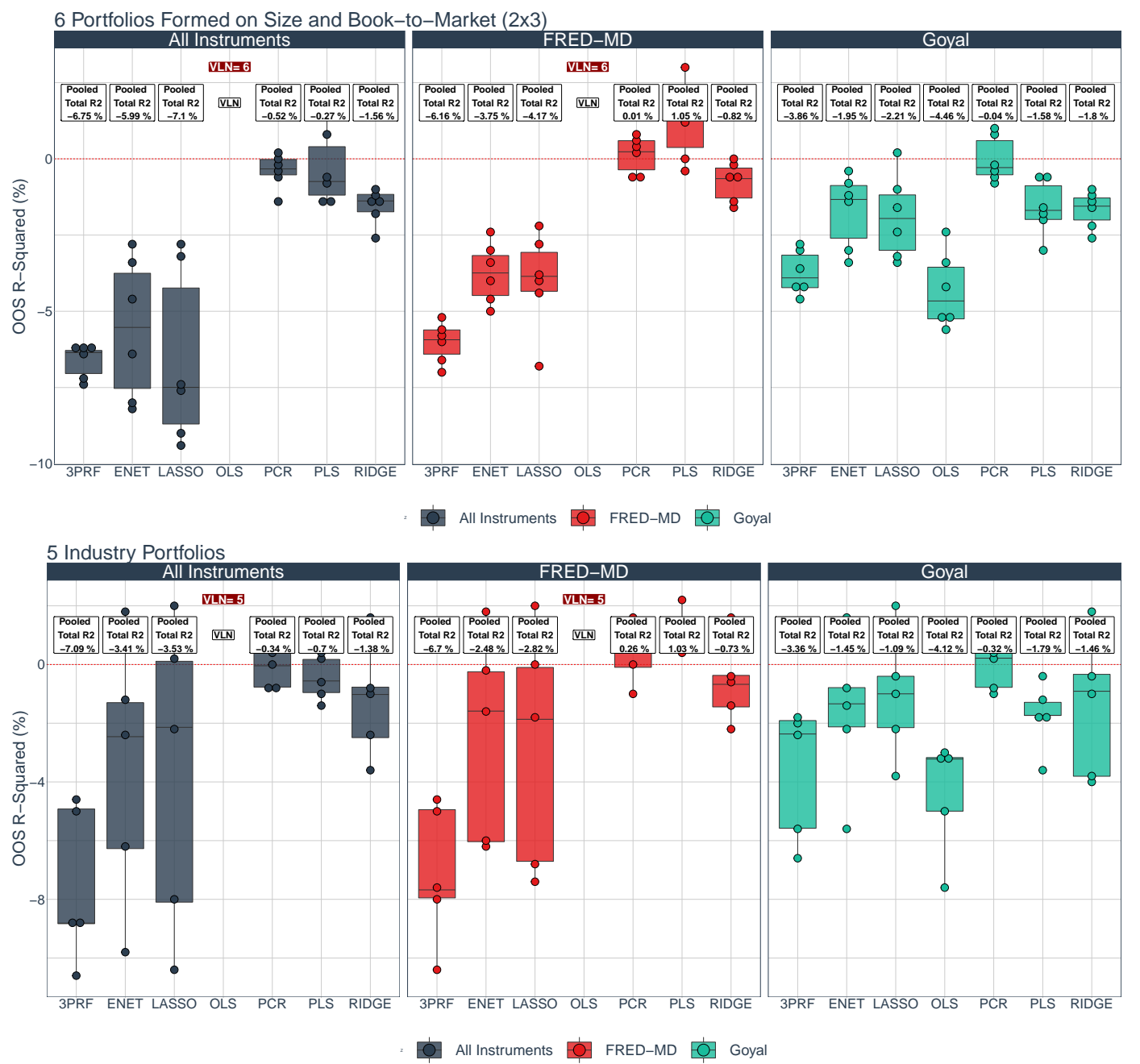

Figure B.5 presents the OOS $R^{2}$ for the datasets with 6 portfolios formed on Size/BTM and the 5 industry portfolios by estimator and set of conditioning information used, along with overlapping boxplots. The pooled OOS $R^{2}$ across all $N$ risky assets in each dataset is also reported in a white box on the top of each plot. In order to make the plots readable and comparable, we filtered the OOS $R^{2}$ larger than -0.5 in absolute values and present the amount of assets that generated these very large numbers (VLN). The amount of VLN per estimator is reported in a red box on top of each plot. 


\section{B.4 Economic Value}

Table B.4: Implied Sharpe Ratios

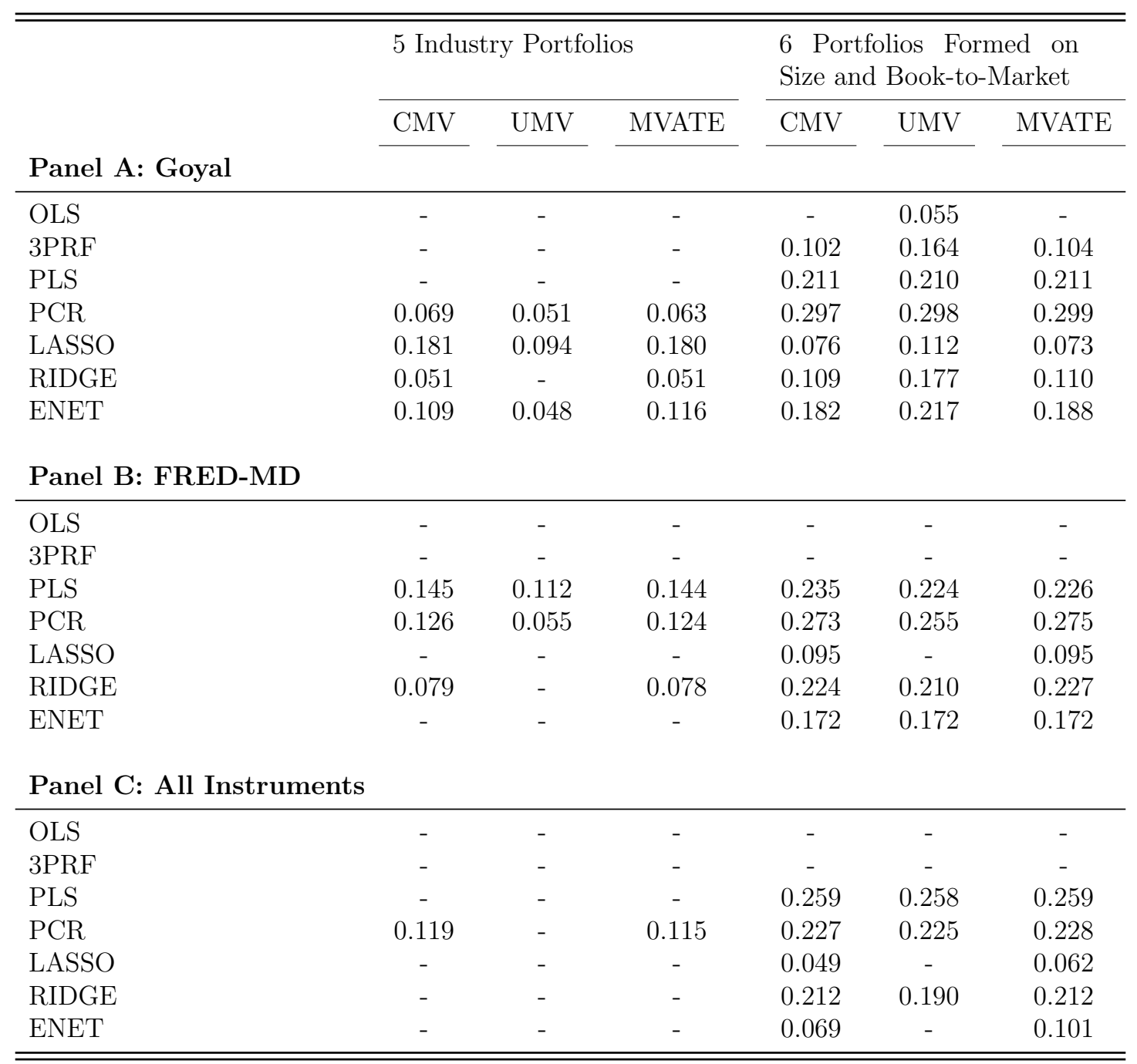

Table B.4 reports the implied $S R^{*}$ for the 5 Industry portfolios and 6 portfolios formed on Size/BTM as given in equation (30) for all seven estimators (OLS, 3PRF, PLS, PCR, LASSO, Ridge and ENet), three different sets of conditioning information (Goyal's, FRED-MD, and "All Instruments", wich is the combination of the previous two with the Economic Policy Uncertainty (EPU) index and the Financial Stress Indicator (FSI)) and three different mean-variance approaches (CMV, UMV, MVATE) to build efficient portfolios. Notice that when the OOS $R^{2}$ is negative, larger absolute $R^{2}$ values than the Sharpe ratio will cause the impossibility of the implied Sharpe ratio to be calculated. These cases are omitted in the table. 


\section{B.4.1 Economic Gains}

Table B.5: Certainty Equivalent Excess Returns (CER) (Monthly \%)

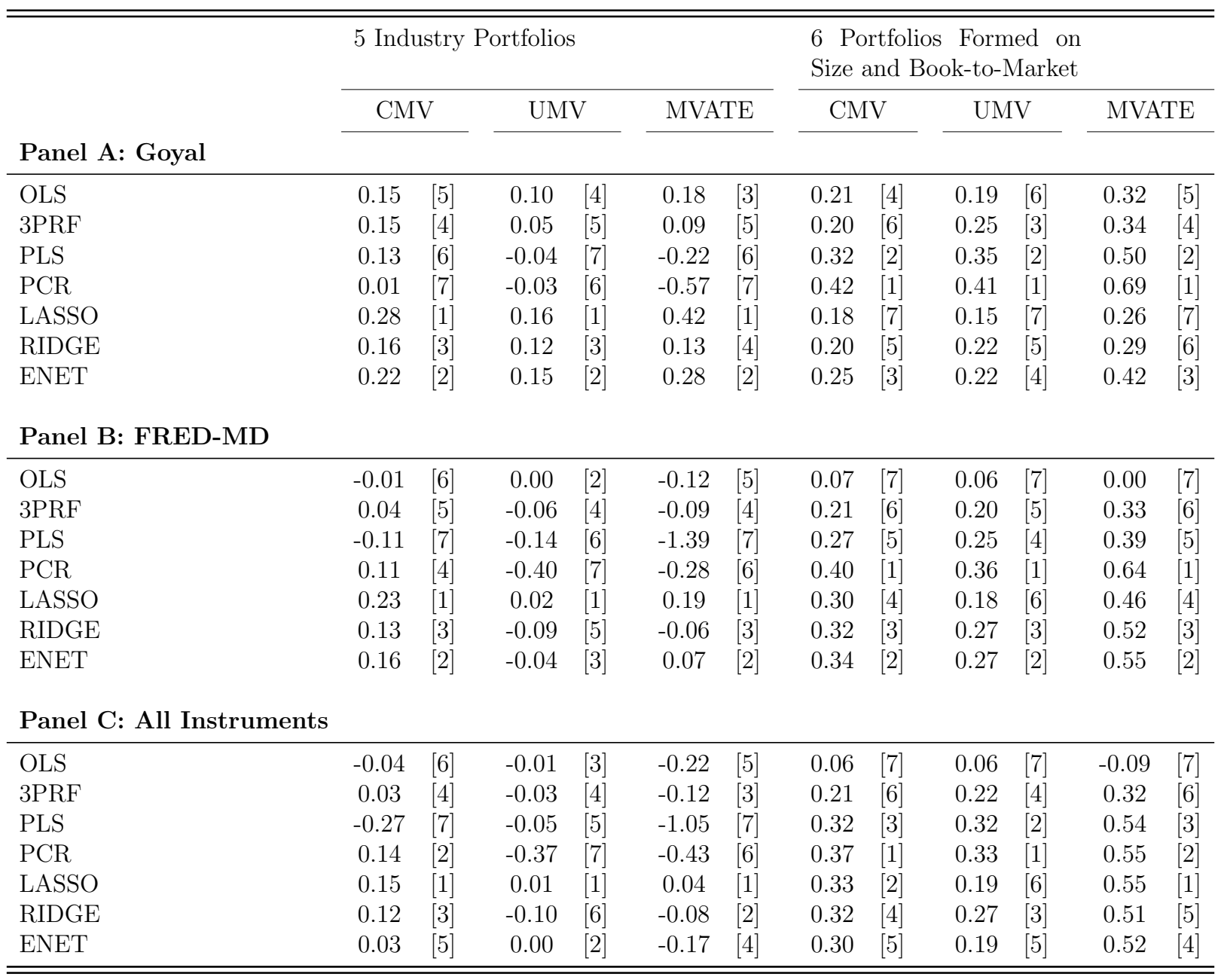

Table B.5 summarises the CER (monthly \%) by estimator and optimal portfolio framework (CMV, UMV and MVATE) for the 5 Industry portfolios and 6 portfolios formed on Size/BTM. Panel A reports the Sharpe ratios generated when the variables from Goyal's website are used as Z. Goyal variables comprises: $b / m, d f r$, $d f y$, infl, ltr, lty, ntis, svar, tms and tbl. Panel B presents the Sharpe ratios obtained using the FRED-MD variables. The FRED-MD is a large dataset containing 128 macroeconomic and financial variables. Finally, panel C shows the Sharpe rations when all variables are used as conditioning information. "All Instruments" is the combination of Goyal and FRED-MD datasets with the Economic Policy Uncertainty (EPU) index and the Financial Stress Indicator (FSI). Inside brackets, we also report the ranking among all 7 estimators within strategy and set of conditioning information employed 
Figure B.6: Management Fee

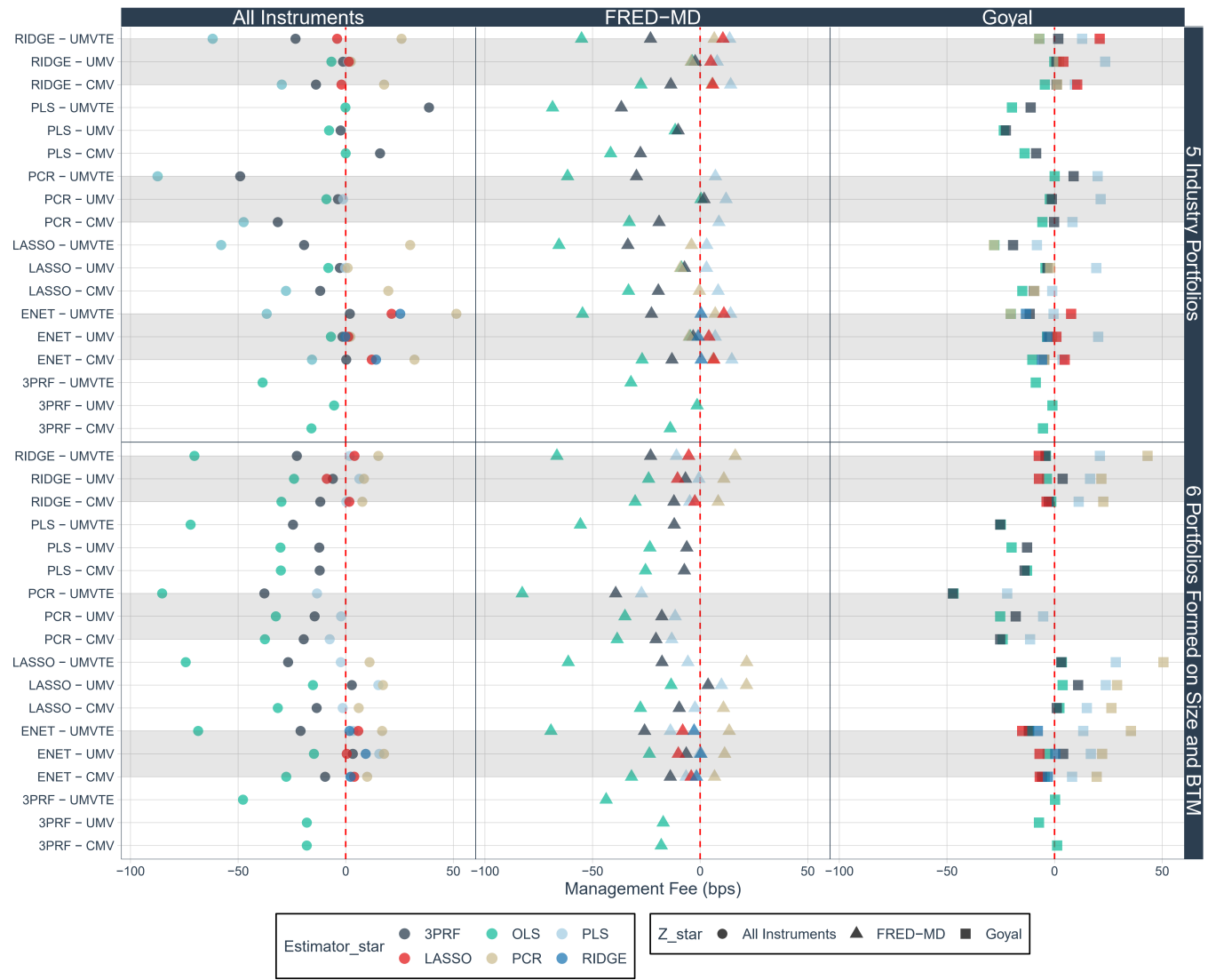

Figure B.6 presents the management fee in bps for the 5 Industry portfolios and 6 portfolios formed on Size/BTM, computed as the solution for $\mathcal{F}$ in equation $(36)$, from an investor switching from an optimal portfolio formed by the estimator and mean-variance framework given in the left axis to another portfolio plotted. The comparison is done in pairs of optimal portfolios generated by each mean-variance strategy (CMV, UMV, and MVATE), estimators (OLS, 3PRF, PLS, PCR, LASSO, Ridge, and ENet) and sets of conditioning information. 


\title{
B.5 Out-of-Sample Analysis - Portfolio Efficiency
}

\author{
Figure B.7: p-values
}
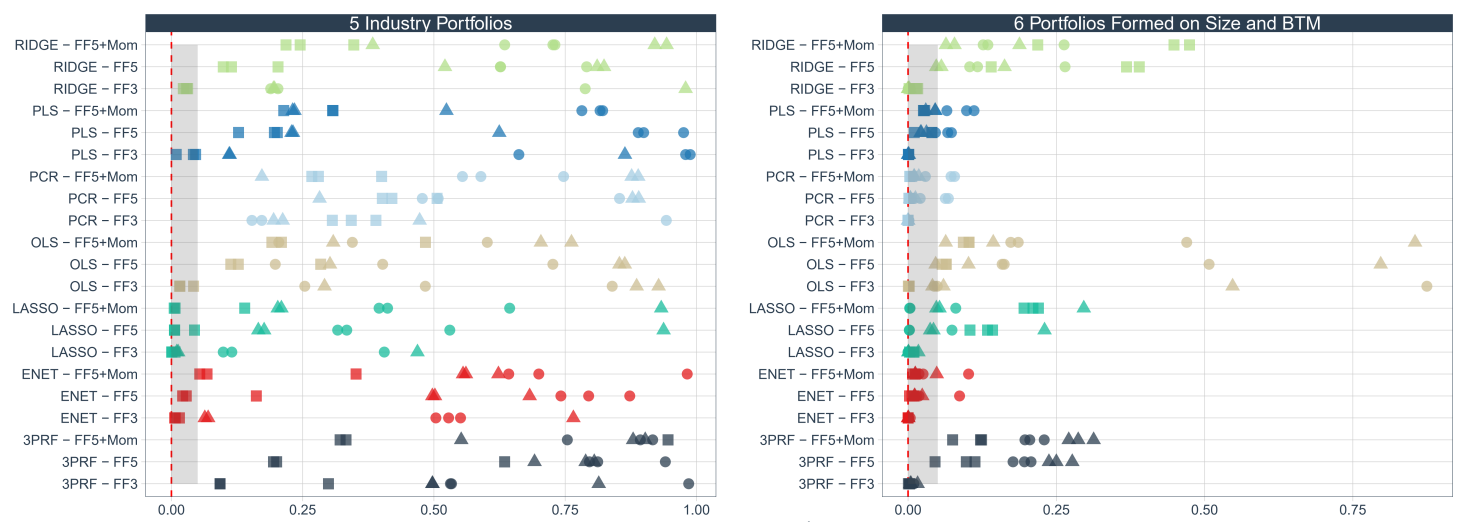

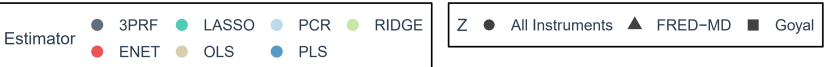

Figure B.7 reports for the 5 Industry portfolios and 6 portfolios formed on Size/BTM the $p$-values of alphas of the regressions of the optimal portfolios generated by each mean-variance strategy (CMV, UMV, and MVATE), estimators (OLS, 3PRF, PLS, PCR, LASSO, Ridge, and ENet) and set of conditioning information used on the Fama-French 3, 5, and $5+$ momentum factor models. The $p$-values are calculated from Newey-West $t$-statistics computed with one lag.

Figure B.8: $R$-Squared
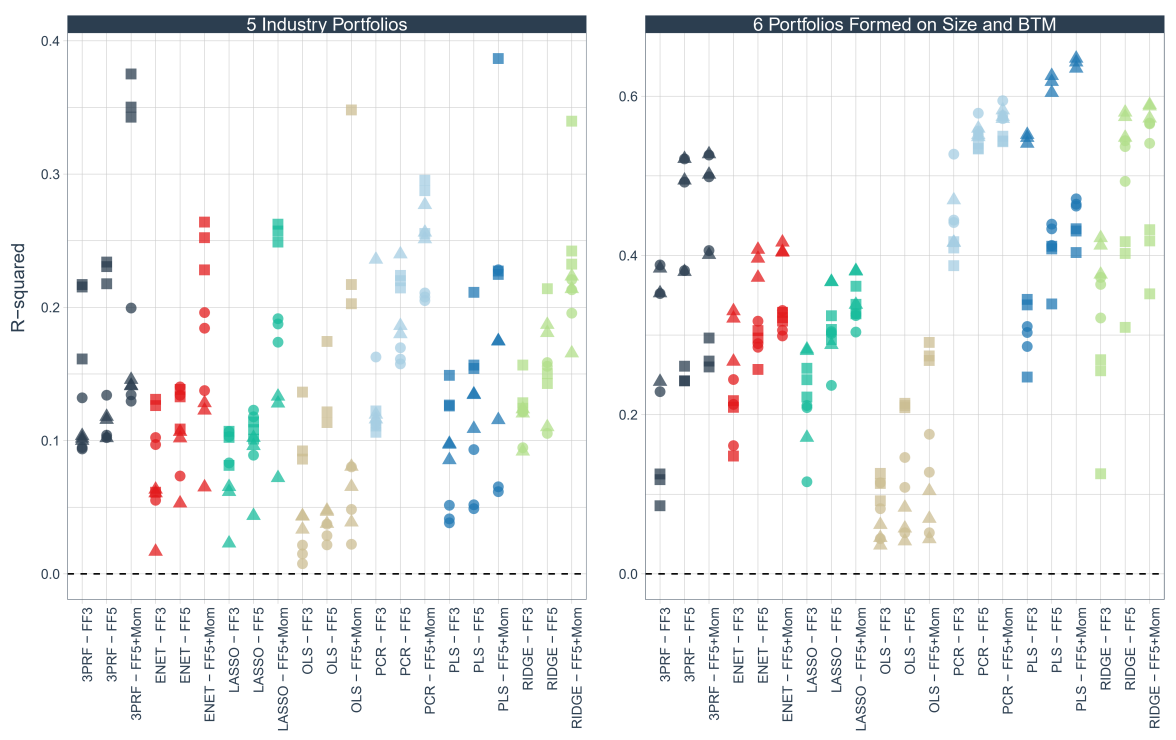

$$
\text { Estimator }- \text { SPRF } \bullet \text { LASSO } \odot \text { PCR } \odot \text { RIDGE }
$$

$Z \bullet$ All Instruments $\triangle$ FRED-MD $\because$ Goyal

Figure B.8 reports for the 5 Industry portfolios and 6 portfolios formed on Size/BTM the $R^{2}$ of the regressions of the optimal portfolios generated by each mean-variance strategy (CMV, UMV, and MVATE), estimators (OLS, 3PRF, PLS, PCR, LASSO, Ridge, and ENet) and set of conditioning information used on the Fama-French 3, 5, and $5+$ momentum factor models. 
Table B.6: Alphas (Monthly \%) - 25 and 100 Portfolios Formed on Size and Book-to-Market - "All Instruments"

\begin{tabular}{|c|c|c|c|c|c|c|c|c|c|c|c|c|c|c|c|c|c|c|c|}
\hline & & \multicolumn{9}{|c|}{25 Portfolios Formed on Size and Book-to-Market } & \multicolumn{9}{|c|}{100 Portfolios Formed on Size and Book-to-Market } \\
\hline & & \multicolumn{3}{|c|}{ CMV } & \multicolumn{3}{|c|}{ UMV } & \multicolumn{3}{|c|}{ MVATE } & \multicolumn{3}{|c|}{ CMV } & \multicolumn{3}{|c|}{ UMV } & \multicolumn{3}{|c|}{ MVATE } \\
\hline & & FF3 & FF5 & $\begin{array}{l}\text { FF5 + } \\
\text { Mom }\end{array}$ & FF3 & FF5 & $\begin{array}{l}\text { FF5 + } \\
\text { Mom }\end{array}$ & FF3 & FF5 & $\begin{array}{l}\text { FF5 + } \\
\text { Mom }\end{array}$ & FF3 & FF5 & $\begin{array}{l}\text { FF5 + } \\
\text { Mom }\end{array}$ & FF3 & FF5 & $\begin{array}{l}\text { FF5 + } \\
\text { Mom }\end{array}$ & FF3 & FF5 & $\begin{array}{l}\text { FF5 }+ \\
\text { Mom }\end{array}$ \\
\hline \multicolumn{20}{|c|}{ Z: All Instruments } \\
\hline \multirow[t]{3}{*}{ OLS } & $\begin{array}{l}\alpha(\%) \\
t \text {-statistics }\end{array}$ & 0.034 & 0.027 & 0.025 & 0.034 & 0.027 & 0.025 & -0.218 & -0.246 & $\begin{array}{l}-0.237 \\
-118]\end{array}$ & 0.018 & 0.016 & 0.015 & 0.018 & 0.016 & 0.015 & $\begin{array}{l}-0.420 \\
-0.791\end{array}$ & $\begin{array}{c}-0.226 \\
-[-0.5]\end{array}$ & $\begin{array}{l}-0.181 \\
{[-0.381}\end{array}$ \\
\hline & $p$-val & $0.002^{* *}$ & $0.027^{*}$ & $0.018^{*}$ & $0.002^{* *}$ & $0.022^{*}$ & $0.017^{*}$ & 0.273 & 0.204 & 0.238 & 0.097 & 0.096 & 0.087 & 0.098 & 0.097 & 0.088 & 0.433 & 0.618 & 0.706 \\
\hline & $R^{2}$ & 0.02 & 0.04 & 0.12 & 0.02 & 0.04 & 0.12 & 0.07 & 0.07 & 0.08 & 0.01 & 0.01 & 0.01 & 0.01 & 0.01 & 0.01 & 0.06 & 0.06 & 0.08 \\
\hline \multirow[t]{4}{*}{$3 \mathrm{PRF}$} & $\alpha(\%)$ & 0.175 & 0.150 & 0.141 & 0.167 & 0.140 & 0.131 & 0.295 & 0.242 & 0.229 & 0.163 & 0.147 & 0.147 & 0.159 & 0.143 & 0.143 & 0.245 & 0.209 & 0.221 \\
\hline & $t$-statistics & [4.01] & {$[3.3]$} & {$[3.4]$} & {$[3.77]$} & {$[2.96]$} & {$[3.24]$} & {$[3.3]$} & {$[2.56]$} & {$[2.66]$} & {$[3.7]$} & {$[2.72]$} & {$[3.01]$} & {$[3.57]$} & {$[2.64]$} & {$[2.91]$} & {$[2.18]$} & {$[1.83]$} & {$[1.84]$} \\
\hline & $p$-val & $0^{* *}$ & $0.001^{* *}$ & $0.001^{* *}$ & $0^{* *}$ & $0.003^{* *}$ & $0.001^{* *}$ & $0.001^{* *}$ & $0.011^{*}$ & $0.008^{* *}$ & $0^{* *}$ & $0.007^{* *}$ & $0.003^{* *}$ & $0^{* *}$ & $0.009^{* *}$ & $0.004^{* *}$ & $0.03^{*}$ & 0.068 & 0.067 \\
\hline & $R^{2}$ & 0.09 & 0.11 & 0.16 & 0.07 & 0.09 & 0.15 & 0.12 & 0.15 & 0.17 & 0.04 & 0.05 & 0.05 & 0.04 & 0.05 & 0.05 & 0.07 & 0.08 & 0.08 \\
\hline \multirow[t]{4}{*}{ PLS } & $\alpha(\%)$ & 0.270 & 0.204 & 0.200 & 0.254 & 0.175 & 0.172 & 0.507 & 0.375 & 0.369 & 0.176 & 0.148 & 0.142 & 0.163 & 0.136 & 0.130 & 0.331 & 0.257 & 0.256 \\
\hline & $t$-statistics & {$[4]$} & {$[3.12]$} & {$[2.93]$} & [3.59] & {$[2.66]$} & {$[2.55]$} & {$[3.7]$} & {$[2.87]$} & {$[2.93]$} & {$[4.36]$} & {$[3.72]$} & {$[3.74]$} & {$[4.36]$} & [3.64] & [3.63] & {$[2.68]$} & {$[2.92]$} & {$[2.75]$} \\
\hline & $p$-val & $0^{* *}$ & $0.002^{* *}$ & $0.004^{* *}$ & $0^{* *}$ & $0.008^{* *}$ & $0.011^{*}$ & $0^{* *}$ & $0.005^{* *}$ & $0.004^{* *}$ & $0^{* *}$ & $0^{* *}$ & $0^{* *}$ & $0^{* *}$ & $0^{* *}$ & $0^{* *}$ & $0.008^{* *}$ & $0.004^{* *}$ & $0.006 * *$ \\
\hline & $R^{2}$ & 0.08 & 0.16 & 0.18 & 0.04 & 0.15 & 0.16 & 0.08 & 0.17 & 0.18 & 0.03 & 0.07 & 0.13 & 0.04 & 0.08 & 0.15 & 0.07 & 0.11 & 0.11 \\
\hline \multirow[t]{4}{*}{ PCR } & $\alpha(\%)$ & 0.332 & 0.278 & 0.259 & 0.301 & 0.265 & 0.247 & 0.652 & 0.542 & 0.506 & 0.267 & 0.251 & 0.246 & 0.249 & 0.232 & 0.227 & 0.533 & 0.498 & 0.489 \\
\hline & $t$-statistics & {$[4.44]$} & [3.87] & {$[3.77]$} & {$[4.1]$} & {$[3.7]$} & [3.85] & {$[4.47]$} & {$[4.03]$} & {$[3.77]$} & {$[3.57]$} & {$[3.3]$} & {$[3.27]$} & [3.62] & {$[3.36]$} & {$[3.3]$} & {$[3.64]$} & {$[3.36]$} & {$[3.3]$} \\
\hline & $p$-val & $0^{* *}$ & $0^{* *}$ & $0^{* *}$ & $0^{* *}$ & $0^{* *}$ & $0^{* *}$ & $0^{* *}$ & $0^{* *}$ & $0^{* *}$ & $0^{* *}$ & $0.001^{* *}$ & $0.001^{* *}$ & $0^{* *}$ & $0.001^{* *}$ & $0.001^{* *}$ & $0^{* *}$ & $0.001^{* *}$ & $0.001^{* *}$ \\
\hline & $R^{2}$ & 0.24 & 0.25 & 0.38 & 0.25 & 0.25 & 0.37 & 0.24 & 0.26 & 0.38 & 0.00 & 0.01 & 0.02 & 0.00 & 0.01 & 0.03 & 0.00 & 0.01 & 0.02 \\
\hline \multirow[t]{4}{*}{ LASSO } & $\alpha(\%)$ & 0.178 & 0.139 & 0.132 & 0.139 & 0.114 & 0.108 & 0.336 & 0.260 & 0.248 & 0.134 & 0.121 & 0.119 & 0.128 & 0.116 & 0.114 & 0.244 & 0.221 & 0.215 \\
\hline & $t$-statistics & {$[3.81]$} & {$[3.22]$} & {$[3.18]$} & [3.65] & {$[3.21]$} & {$[2.98]$} & {$[3.84]$} & {$[3.21]$} & {$[3.21]$} & {$[3.75]$} & {$[3.18]$} & [3.51] & {$[3.72]$} & {$[3.07]$} & {$[3.3]$} & {$[3.3]$} & {$[2.74]$} & {$[2.8]$} \\
\hline & $p$-val & $0^{* *}$ & $0.001^{* *}$ & $0.002^{* *}$ & $0^{* *}$ & $0.002^{* *}$ & $0.003^{* *}$ & $0^{* *}$ & $0.002^{* *}$ & $0.002^{* *}$ & $0^{* *}$ & $0.002^{* *}$ & $0.001^{* *}$ & $0^{* *}$ & $0.002^{* *}$ & $0.001^{* *}$ & $0.001^{* *}$ & $0.007^{* *}$ & $0.0066^{* *}$ \\
\hline & $R^{2}$ & 0.08 & 0.11 & 0.14 & 0.08 & 0.10 & 0.14 & 0.08 & 0.11 & 0.15 & 0.04 & 0.05 & 0.06 & 0.05 & 0.06 & 0.07 & 0.03 & 0.04 & 0.06 \\
\hline \multirow[t]{4}{*}{ RIDGE } & $\alpha(\%)$ & 0.277 & 0.207 & 0.193 & 0.271 & 0.208 & 0.193 & 0.513 & 0.380 & 0.353 & 0.119 & 0.099 & 0.094 & 0.118 & 0.099 & 0.094 & 0.144 & 0.108 & 0.103 \\
\hline & $t$-statistics & [4.31] & {$[2.92]$} & {$[3.32]$} & [3.89] & {$[2.87]$} & {$[3.26]$} & {$[4.37]$} & {$[2.9]$} & {$[2.95]$} & {$[3.4]$} & {$[2.83]$} & {$[2.75]$} & {$[3.38]$} & {$[2.87]$} & {$[2.76]$} & {$[1.34]$} & {$[1.02]$} & {$[1.03]$} \\
\hline & $p$-val & $0^{* *}$ & $0.004^{* *}$ & $0.001^{* *}$ & $0^{* *}$ & $0.004^{* *}$ & $0.001^{* *}$ & $0^{* *}$ & $0.004^{* *}$ & $0.004^{* *}$ & $0.001^{* *}$ & $0.005^{* *}$ & $0.006^{* *}$ & $0.001^{* *}$ & $0.005^{* *}$ & $0.006^{* *}$ & 0.180 & 0.307 & 0.305 \\
\hline & $R^{2}$ & 0.12 & 0.17 & 0.28 & 0.11 & 0.16 & 0.29 & 0.13 & 0.18 & 0.28 & 0.09 & 0.11 & 0.15 & 0.09 & 0.11 & 0.15 & 0.11 & 0.11 & 0.12 \\
\hline \multirow[t]{4}{*}{ ENET } & $\alpha(\%)$ & 0.213 & 0.182 & 0.177 & 0.165 & 0.141 & 0.137 & 0.423 & 0.350 & 0.344 & 0.140 & 0.130 & 0.128 & 0.134 & 0.124 & 0.122 & 0.226 & 0.190 & 0.192 \\
\hline & $t$-statistics & {$[4.79]$} & {$[4.19]$} & {$[4.05]$} & {$[4.13]$} & {$[3.48]$} & {$[3.48]$} & {$[4.64]$} & {$[4]$} & {$[3.98]$} & {$[4.03]$} & {$[3.79]$} & [3.99] & {$[3.9]$} & {$[3.67]$} & [3.85] & {$[2.73]$} & {$[2.14]$} & {$[1.83]$} \\
\hline & $p$-val & $0^{* *}$ & $0^{* *}$ & $0^{* *}$ & $0^{* *}$ & $0.001^{* *}$ & $0.001^{* *}$ & $0^{* *}$ & $0^{* *}$ & $0^{* *}$ & $0^{* *}$ & $0^{* *}$ & $0^{* *}$ & $0^{* *}$ & $0^{* *}$ & $0^{* *}$ & $0.007^{* *}$ & $0.033^{*}$ & 0.069 \\
\hline & $R^{2}$ & 0.10 & 0.12 & 0.15 & 0.08 & 0.10 & 0.13 & 0.06 & 0.09 & 0.11 & 0.05 & 0.06 & 0.08 & 0.06 & 0.07 & 0.09 & 0.01 & 0.02 & 0.03 \\
\hline
\end{tabular}

Table $\mathrm{B} .6$ reports the alphas, $t$-statistics, $p$-values and the $R^{2}$ of the regressions of the optimal portfolios generated by each mean-variance strategy (CMV, UMV, and MVATE), estimators (OLS, 3PRF, PLS, PCR, LASSO, Ridge, and ENet) using the FRED-MD as conditioning information on the Fama-French 3, 5, and $5+$ momentum factor models. "All Instruments" is the combination of Goyal and FRED-MD datasets with the Economic Policy Uncertainty (EPU) index and the Financial Stress Indicator (FSI). Goyal's variables comprises: b/m, dfr, dfy, infl, ltr, lty, ntis, svar, tms and tbl. FRED-MD is a large dataset containing 128 macroeconomic and financial variables. 
Table B.7: Alphas (Monthly \%) - 25 and 100 Portfolios Formed on Size and Book-to-Market - Goyal Variables

\begin{tabular}{|c|c|c|c|c|c|c|c|c|c|c|c|c|c|c|c|c|c|c|c|}
\hline & & \multicolumn{9}{|c|}{25 Portfolios Formed on Size and Book-to-Market } & \multicolumn{9}{|c|}{100 Portfolios Formed on Size and Book-to-Market } \\
\hline & & \multicolumn{3}{|c|}{ CMV } & \multicolumn{3}{|c|}{ UMV } & \multicolumn{3}{|c|}{ MVATE } & \multicolumn{3}{|c|}{ CMV } & \multicolumn{3}{|c|}{ UMV } & \multicolumn{3}{|c|}{ MVATE } \\
\hline & & FF3 & FF5 & $\begin{array}{l}\text { FF5 + } \\
\text { Mom }\end{array}$ & FF3 & FF5 & $\begin{array}{l}\text { FF5 + } \\
\text { Mom }\end{array}$ & FF3 & FF5 & $\begin{array}{l}\text { FF5 + } \\
\text { Mom }\end{array}$ & FF3 & FF5 & $\begin{array}{l}\text { FF5 + } \\
\text { Mom }\end{array}$ & FF3 & FF5 & $\begin{array}{l}\text { FF5 + } \\
\text { Mom }\end{array}$ & FF3 & FF5 & $\begin{array}{l}\text { FF5 + } \\
\text { Mom }\end{array}$ \\
\hline \multicolumn{20}{|c|}{ Z: Goyal } \\
\hline \multirow[t]{4}{*}{ OLS } & $\alpha(\%)$ & 0.199 & 0.184 & 0.169 & 0.193 & 0.176 & 0.163 & 0.329 & 0.309 & 0.287 & 0.076 & 0.072 & 0.065 & 0.074 & 0.070 & 0.064 & -0.023 & 0.002 & 0.004 \\
\hline & $t$-statistics & [5.06] & [4.68] & [4.71] & {$[4.7]$} & [4.42] & [4.25] & {$[4.18]$} & {$[3.92]$} & [4.03] & {$[1.59]$} & {$[1.82]$} & {$[1.54]$} & {$[1.56]$} & {$[1.8]$} & {$[1.52]$} & {$[-0.16]$} & {$[0.01]$} & {$[0.03]$} \\
\hline & $p$-val & $0^{* *}$ & $0^{* *}$ & $0^{* *}$ & $0^{* *}$ & $0^{* *}$ & $0^{* *}$ & $0^{* *}$ & $0^{* *}$ & $0^{* *}$ & 0.112 & 0.070 & 0.124 & 0.120 & 0.073 & 0.128 & 0.873 & 0.989 & 0.974 \\
\hline & $R^{2}$ & 0.01 & 0.05 & 0.18 & 0.02 & 0.06 & 0.18 & 0.01 & 0.05 & 0.14 & 0.01 & 0.03 & 0.04 & 0.01 & 0.03 & 0.04 & 0.01 & 0.02 & 0.02 \\
\hline \multirow[t]{4}{*}{ 3PRF } & $\alpha(\%)$ & 0.226 & 0.210 & 0.194 & 0.244 & 0.224 & 0.210 & 0.403 & 0.381 & 0.357 & 0.143 & 0.128 & 0.121 & 0.146 & 0.129 & 0.122 & 0.134 & 0.102 & 0.101 \\
\hline & $t$-statistics & [4.99] & [5.06] & {$[4.97]$} & {$[4.89]$} & {$[4.71]$} & [4.53] & {$[4.63]$} & {$[4.54]$} & [4.61] & {$[2.46]$} & {$[3.04]$} & {$[2.74]$} & {$[2.68]$} & {$[3.29]$} & {$[2.99]$} & {$[0.98]$} & {$[0.92]$} & {$[0.91]$} \\
\hline & $p$-val & $0^{* *}$ & $0^{* *}$ & $0^{* *}$ & $0^{* *}$ & $0^{* *}$ & $0^{* *}$ & $0^{* *}$ & $0^{* *}$ & $0^{* *}$ & $0.015^{*}$ & $0.003^{* *}$ & $0.007 * *$ & $0.008^{* *}$ & $0.001 * *$ & $0.003^{* *}$ & 0.328 & 0.358 & 0.363 \\
\hline & $R^{2}$ & 0.03 & 0.06 & 0.19 & 0.03 & 0.07 & 0.18 & 0.02 & 0.05 & 0.14 & 0.01 & 0.02 & 0.03 & 0.01 & 0.02 & 0.03 & 0.01 & 0.02 & 0.02 \\
\hline \multirow[t]{4}{*}{ PLS } & $\alpha(\%)$ & 0.292 & 0.254 & 0.235 & 0.352 & 0.303 & 0.285 & 0.537 & 0.472 & 0.435 & 0.242 & 0.217 & 0.206 & 0.243 & 0.217 & 0.207 & 0.462 & 0.421 & 0.403 \\
\hline & $t$-statistics & {$[4.35]$} & {$[3.78]$} & {$[3.51]$} & {$[3.73]$} & {$[3.52]$} & {$[3.15]$} & [4.64] & {$[3.87]$} & {$[3.78]$} & {$[3.9]$} & {$[3.97]$} & {$[3.67]$} & {$[3.85]$} & {$[3.96]$} & {$[3.62]$} & {$[4.38]$} & [3.97] & [4.14] \\
\hline & $p$-val & $0^{* *}$ & $0^{* *}$ & $0.001^{* *}$ & $0^{* *}$ & $0.001 * *$ & $0.002^{* *}$ & $0^{* *}$ & $0^{* *}$ & $0^{* *}$ & $0^{* *}$ & $0^{* *}$ & $0^{* *}$ & $0^{* *}$ & $0^{* *}$ & $0^{* *}$ & $0^{* *}$ & $0^{* *}$ & $0^{* *}$ \\
\hline & $R^{2}$ & 0.11 & 0.13 & 0.22 & 0.09 & 0.12 & 0.20 & 0.12 & 0.13 & 0.23 & 0.01 & 0.02 & 0.05 & 0.01 & 0.02 & 0.05 & 0.02 & 0.02 & 0.05 \\
\hline \multirow[t]{4}{*}{ PCR } & $\alpha(\%)$ & 0.367 & 0.326 & 0.311 & 0.381 & 0.329 & 0.318 & 0.700 & 0.624 & 0.594 & 0.277 & 0.267 & 0.260 & 0.272 & 0.259 & 0.252 & 0.529 & 0.510 & 0.496 \\
\hline & $t$-statistics & {$[5.38]$} & {$[4.64]$} & {$[4.52]$} & {$[4.62]$} & {$[4.07]$} & {$[3.98]$} & {$[5.36]$} & {$[4.65]$} & {$[4.56]$} & {$[4.21]$} & {$[3.98]$} & {$[3.73]$} & {$[4.21]$} & {$[3.78]$} & {$[3.77]$} & {$[4.23]$} & {$[3.83]$} & {$[3.83]$} \\
\hline & $p$-val & $0^{* *}$ & $0^{* *}$ & $0^{* *}$ & $0^{* *}$ & $0^{* *}$ & $0^{* *}$ & $0^{* *}$ & $0^{* *}$ & $0^{* *}$ & $0^{* *}$ & $0^{* *}$ & $0^{* *}$ & $0^{* *}$ & $0^{* *}$ & $0^{* *}$ & $0^{* *}$ & $0^{* *}$ & $0^{* *}$ \\
\hline & $R^{2}$ & 0.17 & 0.19 & 0.24 & 0.13 & 0.16 & 0.19 & 0.18 & 0.20 & 0.25 & 0.01 & 0.02 & 0.03 & 0.01 & 0.02 & 0.04 & 0.02 & 0.03 & 0.04 \\
\hline \multirow[t]{4}{*}{ LASSO } & $\alpha(\%)$ & 0.169 & 0.159 & 0.151 & 0.151 & 0.140 & 0.133 & 0.326 & 0.305 & 0.291 & 0.089 & 0.079 & 0.079 & 0.092 & 0.081 & 0.080 & 0.164 & 0.136 & 0.131 \\
\hline & $t$-statistics & [4.09] & {$[4.29]$} & {$[3.75]$} & {$[4.43]$} & {$[4.45]$} & [4.07] & {$[4.04]$} & {$[4.51]$} & {$[3.8]$} & {$[1.71]$} & {$[1.71]$} & {$[1.76]$} & {$[1.83]$} & {$[1.94]$} & {$[1.94]$} & {$[1.57]$} & {$[1.61]$} & {$[1.55]$} \\
\hline & $p$-val & $0^{* *}$ & $0^{* *}$ & $0^{* *}$ & $0^{* *}$ & $0^{* *}$ & $0^{* *}$ & $0^{* *}$ & $0^{* *}$ & $0^{* *}$ & 0.088 & 0.089 & 0.080 & 0.069 & 0.054 & 0.053 & 0.118 & 0.108 & 0.123 \\
\hline & $R^{2}$ & 0.06 & 0.07 & 0.11 & 0.07 & 0.08 & 0.13 & 0.07 & 0.08 & 0.12 & 0.01 & 0.01 & 0.01 & 0.00 & 0.01 & 0.01 & 0.01 & 0.02 & 0.02 \\
\hline \multirow[t]{4}{*}{ RIDGE } & $\alpha(\%)$ & 0.258 & 0.232 & 0.214 & 0.239 & 0.213 & 0.199 & 0.456 & 0.408 & 0.375 & 0.092 & 0.092 & 0.089 & 0.104 & 0.103 & 0.100 & 0.120 & 0.119 & 0.117 \\
\hline & $t$-statistics & {$[5.67]$} & [5.29] & {$[5.25]$} & {$[5.3]$} & {$[5.16]$} & [4.86] & {$[5.3]$} & {$[4.95]$} & [5.31] & {$[1.21]$} & {$[1.38]$} & {$[1.39]$} & {$[1.58]$} & {$[1.81]$} & {$[1.77]$} & {$[0.77]$} & {$[0.9]$} & {$[0.87]$} \\
\hline & $p$-val & $0^{* *}$ & $0^{* *}$ & $0^{* *}$ & $0^{* *}$ & $0^{* *}$ & $0^{* *}$ & $0^{* *}$ & $0^{* *}$ & $0^{* *}$ & 0.225 & 0.169 & 0.166 & 0.116 & 0.072 & 0.077 & 0.444 & 0.366 & 0.385 \\
\hline & $R^{2}$ & 0.05 & 0.07 & 0.19 & 0.08 & 0.11 & 0.22 & 0.05 & 0.07 & 0.19 & 0.01 & 0.02 & 0.02 & 0.02 & 0.02 & 0.02 & 0.01 & 0.01 & 0.01 \\
\hline \multirow[t]{4}{*}{ ENET } & $\alpha(\%)$ & 0.192 & 0.179 & 0.168 & 0.176 & 0.157 & 0.148 & 0.329 & 0.300 & 0.283 & 0.094 & 0.085 & 0.085 & 0.095 & 0.084 & 0.085 & 0.076 & 0.052 & 0.060 \\
\hline & $t$-statistics & [5.49] & [5.47] & {$[5]$} & {$[5.42]$} & [5.61] & [5.17] & {$[4.1]$} & {$[4.02]$} & {$[3.9]$} & {$[1.66]$} & {$[1.8]$} & {$[1.88]$} & {$[1.73]$} & {$[1.9]$} & {$[1.99]$} & {$[0.53]$} & {$[0.46]$} & {$[0.53]$} \\
\hline & $p$-val & $0^{* *}$ & $0^{* *}$ & $0^{* *}$ & $0^{* *}$ & $0^{* *}$ & $0^{* *}$ & $0^{* *}$ & $0^{* *}$ & $0^{* *}$ & 0.099 & 0.073 & 0.061 & 0.084 & 0.059 & $0.047^{*}$ & 0.596 & 0.646 & 0.595 \\
\hline & $R^{2}$ & 0.04 & 0.06 & 0.14 & 0.06 & 0.09 & 0.17 & 0.04 & 0.06 & 0.11 & 0.01 & 0.01 & 0.01 & 0.01 & 0.01 & 0.01 & 0.00 & 0.01 & 0.01 \\
\hline
\end{tabular}

Table $\quad$ B.7 reports the alphas, $t$-statistics, $p$-values and the $R^{2}$ of the regressions of the optimal portfolios generated by each mean-variance strategy (CMV, UMV, and MVATE), estimators (OLS, 3PRF, PLS, PCR, LASSO, Ridge, and ENet) using Goyal's variables as conditioning information on the Fama-French 3, 5, and $5+$ momentum factor models. Goyal's variables comprises: b/m, dfr, dfy, infl, ltr, lty, ntis, svar, tms and tbl. 
Table B.8: Alphas from Factor Models (Monthly \%) - 5 Industry Portfolios and 6 Portfolios Formed on Size and Book-to-Market - FRED-MD

\begin{tabular}{|c|c|c|c|c|c|c|c|c|c|c|c|c|c|c|c|c|c|c|c|}
\hline & & \multicolumn{9}{|c|}{5 Industry Portfolios } & \multicolumn{9}{|c|}{6 Portfolios Formed on Size and Book-to-Market } \\
\hline & & \multicolumn{3}{|c|}{ CMV } & \multicolumn{3}{|c|}{ UMV } & \multicolumn{3}{|c|}{ MVATE } & \multicolumn{3}{|c|}{ CMV } & \multicolumn{3}{|c|}{ UMV } & \multicolumn{3}{|c|}{ MVATE } \\
\hline & & FF3 & FF5 & $\begin{array}{l}\text { FF5 + } \\
\text { Mom }\end{array}$ & FF3 & FF5 & $\begin{array}{l}\text { FF5 + } \\
\text { Mom }\end{array}$ & FF3 & FF5 & $\begin{array}{l}\text { FF5 + } \\
\text { Mom }\end{array}$ & FF3 & FF5 & $\begin{array}{l}\text { FF5 + } \\
\text { Mom }\end{array}$ & FF3 & FF5 & $\begin{array}{l}\text { FF5 + } \\
\text { Mom }\end{array}$ & FF3 & FF5 & $\begin{array}{l}\text { FF5 + } \\
\text { Mom }\end{array}$ \\
\hline \multicolumn{20}{|c|}{ Z: FRED-MD } \\
\hline \multirow[t]{4}{*}{ OLS } & $\alpha(\%)$ & -0.006 & -0.007 & -0.012 & -0.003 & -0.006 & -0.011 & -0.090 & -0.092 & -0.095 & 0.070 & 0.062 & 0.058 & 0.062 & 0.048 & 0.044 & 0.039 & 0.018 & 0.013 \\
\hline & $t$-statistics & {$[-0.14]$} & {$[-0.17]$} & {$[-0.3]$} & {$[-0.09]$} & {$[-0.19]$} & {$[-0.38]$} & {$[-1.06]$} & {$[-1.03]$} & {$[-1.02]$} & {$[2.05]$} & {$[2]$} & [1.86] & [1.89] & [1.64] & {$[1.47]$} & {$[0.6]$} & {$[0.26]$} & {$[0.18]$} \\
\hline & $p$-val & 0.886 & 0.864 & 0.762 & 0.928 & 0.853 & 0.704 & 0.292 & 0.302 & 0.308 & $0.041^{*}$ & $0.047^{*}$ & 0.063 & 0.060 & 0.102 & 0.144 & 0.547 & 0.797 & 0.855 \\
\hline & $R^{2}$ & 0.04 & 0.05 & 0.07 & 0.04 & 0.05 & 0.08 & 0.03 & 0.04 & 0.04 & 0.05 & 0.06 & 0.07 & 0.06 & 0.08 & 0.10 & 0.04 & 0.04 & 0.04 \\
\hline \multirow[t]{4}{*}{$3 \mathrm{PRF}$} & $\alpha(\%)$ & 0.091 & 0.039 & 0.019 & -0.025 & -0.045 & -0.066 & 0.178 & 0.083 & 0.046 & 0.210 & 0.074 & 0.067 & 0.203 & 0.082 & 0.071 & 0.399 & 0.143 & 0.130 \\
\hline & $t$-statistics & {$[0.68]$} & {$[0.25]$} & {$[0.12]$} & {$[-0.24]$} & {$[-0.4]$} & {$[-0.6]$} & {$[0.68]$} & {$[0.27]$} & {$[0.15]$} & {$[2.84]$} & {$[1.15]$} & {$[1.07]$} & {$[2.42]$} & [1.09] & {$[1.01]$} & {$[2.91]$} & {$[1.18]$} & {$[1.1]$} \\
\hline & $p$-val & 0.497 & 0.805 & 0.902 & 0.814 & 0.692 & 0.552 & 0.497 & 0.789 & 0.879 & $0.005^{* *}$ & 0.250 & 0.287 & $0.016^{*}$ & 0.277 & 0.313 & $0.004^{* *}$ & 0.238 & 0.271 \\
\hline & $R^{2}$ & 0.10 & 0.12 & 0.15 & 0.10 & 0.10 & 0.14 & 0.10 & 0.12 & 0.14 & 0.35 & 0.49 & 0.50 & 0.24 & 0.38 & 0.40 & 0.38 & 0.52 & 0.53 \\
\hline \multirow[t]{4}{*}{ PLS } & $\alpha(\%)$ & 0.484 & 0.495 & 0.434 & 0.035 & -0.111 & -0.128 & 0.943 & 0.974 & 0.856 & 0.275 & 0.153 & 0.141 & 0.235 & 0.133 & 0.113 & 0.520 & 0.285 & 0.260 \\
\hline & $t$-statistics & {$[1.6]$} & {$[1.2]$} & {$[1.2]$} & {$[0.17]$} & {$[-0.49]$} & {$[-0.64]$} & {$[1.6]$} & [1.21] & {$[1.19]$} & {$[3.74]$} & {$[2.3]$} & {$[2.01]$} & {$[3.25]$} & {$[2.32]$} & {$[2.18]$} & {$[3.73]$} & {$[2.17]$} & {$[2.01]$} \\
\hline & $p$-val & 0.110 & 0.232 & 0.231 & 0.864 & 0.624 & 0.524 & 0.111 & 0.229 & 0.234 & $0^{* *}$ & $0.022^{*}$ & $0.046^{*}$ & $0.001 * *$ & $0.021^{*}$ & $0.03^{*}$ & $0^{* *}$ & $0.031^{*}$ & $0.046^{*}$ \\
\hline & $R^{2}$ & 0.10 & 0.13 & 0.17 & 0.09 & 0.11 & 0.12 & 0.10 & 0.13 & 0.17 & 0.54 & 0.62 & 0.63 & 0.55 & 0.60 & 0.65 & 0.55 & 0.63 & 0.64 \\
\hline \multirow[t]{4}{*}{ PCR } & $\alpha(\%)$ & 0.251 & 0.028 & -0.024 & -0.150 & -0.196 & -0.248 & 0.468 & 0.050 & -0.053 & 0.393 & 0.219 & 0.203 & 0.324 & 0.186 & 0.171 & 0.771 & 0.434 & 0.403 \\
\hline & $t$-statistics & {$[1.3]$} & {$[0.15]$} & {$[-0.14]$} & {$[-0.72]$} & {$[-1.08]$} & {$[-1.37]$} & {$[1.25]$} & {$[0.14]$} & {$[-0.16]$} & {$[4.3]$} & {$[2.9]$} & {$[2.58]$} & [4.37] & {$[2.52]$} & {$[2.38]$} & {$[4.27]$} & {$[2.92]$} & {$[2.59]$} \\
\hline & $p$-val & 0.195 & 0.878 & 0.889 & 0.473 & 0.282 & 0.172 & 0.212 & 0.890 & 0.877 & $0^{* *}$ & $0.004^{* *}$ & $0.011^{*}$ & $0^{* *}$ & $0.012^{*}$ & $0.018^{*}$ & $0^{* *}$ & $0.004^{* *}$ & $0.01^{*}$ \\
\hline & $R^{2}$ & 0.12 & 0.19 & 0.26 & 0.24 & 0.24 & 0.28 & 0.12 & 0.18 & 0.25 & 0.42 & 0.55 & 0.57 & 0.47 & 0.56 & 0.58 & 0.42 & 0.55 & 0.57 \\
\hline \multirow[t]{4}{*}{ LASSO } & $\alpha(\%)$ & 0.310 & 0.201 & 0.176 & 0.081 & 0.010 & -0.010 & 0.575 & 0.377 & 0.330 & 0.283 & 0.165 & 0.154 & 0.168 & 0.074 & 0.061 & 0.548 & 0.325 & 0.303 \\
\hline & $t$-statistics & [2.59] & {$[1.39]$} & {$[1.28]$} & {$[0.73]$} & {$[0.08]$} & {$[-0.08]$} & {$[2.51]$} & [1.35] & {$[1.26]$} & {$[3.47]$} & {$[2.04]$} & [1.94] & {$[2.39]$} & {$[1.2]$} & [1.05] & {$[3.49]$} & {$[2.09]$} & {$[1.99]$} \\
\hline & $p$-val & $0.01^{*}$ & 0.166 & 0.203 & 0.469 & 0.937 & 0.933 & $0.013^{*}$ & 0.177 & 0.210 & $0.001^{* *}$ & $0.043^{*}$ & 0.053 & $0.017^{*}$ & 0.230 & 0.296 & $0.001^{* *}$ & $0.037^{*}$ & $0.048^{*}$ \\
\hline & $R^{2}$ & 0.07 & 0.10 & 0.13 & 0.02 & 0.04 & 0.07 & 0.06 & 0.10 & 0.13 & 0.28 & 0.37 & 0.38 & 0.17 & 0.29 & 0.34 & 0.28 & 0.37 & 0.38 \\
\hline \multirow[t]{4}{*}{ RIDGE } & $\alpha(\%)$ & 0.218 & 0.043 & 0.012 & -0.004 & -0.095 & -0.131 & 0.417 & 0.090 & 0.034 & 0.305 & 0.133 & 0.122 & 0.250 & 0.098 & 0.086 & 0.596 & 0.272 & 0.254 \\
\hline & $t$-statistics & {$[1.3]$} & {$[0.22]$} & {$[0.07]$} & {$[-0.03]$} & {$[-0.64]$} & {$[-0.87]$} & {$[1.3]$} & {$[0.24]$} & {$[0.1]$} & [3.97] & [1.92] & {$[1.77]$} & {$[3.15]$} & {$[1.4]$} & {$[1.32]$} & {$[3.96]$} & {$[1.99]$} & [1.86] \\
\hline & $p$-val & 0.196 & 0.824 & 0.943 & 0.979 & 0.521 & 0.383 & 0.194 & 0.811 & 0.921 & $0^{* *}$ & 0.056 & 0.078 & $0.002^{* *}$ & 0.163 & 0.188 & $0^{* *}$ & $0.048^{*}$ & 0.064 \\
\hline & $R^{2}$ & 0.12 & 0.19 & 0.22 & 0.09 & 0.11 & 0.17 & 0.12 & 0.18 & 0.21 & 0.41 & 0.57 & 0.59 & 0.38 & 0.55 & 0.57 & 0.42 & 0.58 & 0.59 \\
\hline \multirow[t]{4}{*}{ ENET } & $\alpha(\%)$ & 0.246 & 0.119 & 0.099 & 0.037 & -0.056 & -0.070 & 0.466 & 0.235 & 0.196 & 0.313 & 0.203 & 0.194 & 0.254 & 0.151 & 0.139 & 0.606 & 0.399 & 0.383 \\
\hline & $t$-statistics & {$[1.86]$} & {$[0.67]$} & {$[0.58]$} & {$[0.3]$} & {$[-0.41]$} & {$[-0.49]$} & {$[1.82]$} & {$[0.68]$} & {$[0.59]$} & {$[3.82]$} & {$[2.57]$} & {$[2.53]$} & {$[3.61]$} & {$[2.27]$} & {$[1.98]$} & {$[3.72]$} & {$[2.55]$} & {$[2.53]$} \\
\hline & $p$-val & 0.064 & 0.502 & 0.561 & 0.766 & 0.682 & 0.623 & 0.070 & 0.497 & 0.556 & $0^{* *}$ & $0.011^{*}$ & $0.012^{*}$ & $0^{* *}$ & $0.024^{*}$ & $0.048^{*}$ & $0^{* * *}$ & $0.011^{*}$ & $0.012^{*}$ \\
\hline & $R^{2}$ & 0.06 & 0.11 & 0.13 & 0.02 & 0.05 & 0.07 & 0.06 & 0.10 & 0.12 & 0.33 & 0.41 & 0.42 & 0.27 & 0.37 & 0.40 & 0.32 & 0.40 & 0.40 \\
\hline
\end{tabular}

Table B.8 reports the alphas, $t$-statistics, $p$-values and the $R^{2}$ of the regressions of the optimal portfolios generated by each mean-variance strategy (CMV, UMV, and MVATE), estimators (OLS, 3PRF, PLS, PCR, LASSO, Ridge, and ENet) using the FRED-MD as conditioning information on the Fama-French 3, 5, and $5+$ momentum factor models. FRED-MD is a large dataset containing 128 macroeconomic and financial variables. 
Table B.9: Alphas (Monthly \%) - 5 Industry Portfolios and 6 Portfolios Formed on Size and Book-to-Market - "All Instruments"

\begin{tabular}{|c|c|c|c|c|c|c|c|c|c|c|c|c|c|c|c|c|c|c|c|}
\hline & & \multicolumn{9}{|c|}{5 Industry Portfolios } & \multicolumn{9}{|c|}{6 Portfolios Formed on Size and Book-to-Market } \\
\hline & & \multicolumn{3}{|c|}{ CMV } & \multicolumn{3}{|c|}{ UMV } & \multicolumn{3}{|c|}{ MVATE } & \multicolumn{3}{|c|}{ CMV } & \multicolumn{3}{|c|}{ UMV } & \multicolumn{3}{|c|}{ MVATE } \\
\hline & & FF3 & FF5 & $\begin{array}{l}\text { FF5 + } \\
\text { Mom }\end{array}$ & FF3 & FF5 & 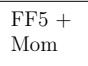 & FF3 & FF5 & $\begin{array}{l}\text { FF5 + } \\
\text { Mom }\end{array}$ & FF3 & FF5 & $\begin{array}{l}\text { FF5 + } \\
\text { Mom }\end{array}$ & FF3 & FF5 & $\begin{array}{l}\text { FF5 + } \\
\text { Mom }\end{array}$ & FF3 & FF5 & $\begin{array}{l}\text { FF5 + } \\
\text { Mom }\end{array}$ \\
\hline \multicolumn{20}{|c|}{ Z: All Instruments } \\
\hline \multirow[t]{3}{*}{ OLS } & $\begin{array}{l}\alpha(\%) \\
t \text {-statistics }\end{array}$ & $\begin{array}{c}-0.032 \\
{[-0.7]}\end{array}$ & $\begin{array}{l}-0.042 \\
{[-0.84]}\end{array}$ & $\begin{array}{l}-0.047 \\
{[-0.95]}\end{array}$ & $\begin{array}{c}-0.006 \\
-0.2]\end{array}$ & $\begin{array}{l}-0.011 \\
{[-0.35]}\end{array}$ & $\begin{array}{l}-0.015 \\
{[-0.52]}\end{array}$ & $\begin{array}{c}-0.162 \\
{[-1.14]}\end{array}$ & $\begin{array}{l}-0.176 \\
{[-1.29]}\end{array}$ & $\begin{array}{l}-0.178 \\
{[-1.27]}\end{array}$ & $\begin{array}{l}0.064 \\
{[1.987}\end{array}$ & $\begin{array}{l}0.046 \\
{[1.41]}\end{array}$ & $\begin{array}{l}0.044 \\
{[1.37]}\end{array}$ & $\begin{array}{l}0.063 \\
{[2.02]}\end{array}$ & $\begin{array}{l}0.044 \\
{[1.4]}\end{array}$ & $\begin{array}{l}0.041 \\
{[1.33]}\end{array}$ & $\begin{array}{l}-0.016 \\
{[-0.16]}\end{array}$ & $\begin{array}{l}-0.070 \\
-0.066\end{array}$ & $\begin{array}{l}-0.070 \\
-0.072]\end{array}$ \\
\hline & ${ }_{p \text {-vatistics }}^{p-v a l}$ & $\begin{array}{l}{[-0.7]} \\
0.484\end{array}$ & $\begin{array}{r}{[-0.840} \\
0.402\end{array}$ & 0.345 & $\begin{array}{l}{[-0.2]} \\
0.840\end{array}$ & $\begin{array}{c}{[-0.35]} \\
0.727\end{array}$ & $\begin{array}{l}{[-0.52]} \\
0.602\end{array}$ & $\begin{array}{c}{[-1.144]} \\
0.254\end{array}$ & $\begin{array}{r}{[-1.29]} \\
0.198\end{array}$ & 0.205 & $0.049^{*}$ & [1.41] & 0.173 & $0.044^{*}$ & $\begin{array}{l}1.162 \\
0.162\end{array}$ & [1.183] & $\begin{array}{l}{[-0.16]} \\
0.875\end{array}$ & $\begin{array}{c}{[-0.606} \\
0.508\end{array}$ & 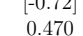 \\
\hline & $R^{2}$ & 0.01 & 0.03 & 0.05 & 0.02 & 0.04 & 0.08 & 0.01 & 0.02 & 0.02 & 0.08 & 0.11 & 0.13 & 0.11 & 0.15 & 0.18 & 0.04 & 0.05 & 0.05 \\
\hline \multirow[t]{4}{*}{$3 \mathrm{PRF}$} & $\alpha(\%)$ & 0.086 & 0.037 & 0.016 & 0.002 & -0.009 & -0.037 & 0.168 & 0.080 & 0.041 & 0.213 & 0.086 & 0.079 & 0.219 & 0.096 & 0.084 & 0.393 & 0.154 & 0.143 \\
\hline & $t$-statistics & {$[0.63]$} & [0.24] & {$[0.1]$} & {$[0.02]$} & {$[-0.07]$} & {$[-0.31]$} & {$[0.62]$} & {$[0.26]$} & {$[0.13]$} & {$[3.05]$} & {$[1.35]$} & {$[1.29]$} & {$[2.6]$} & {$[1.26]$} & {$[1.2]$} & {$[3.05]$} & {$[1.3]$} & {$[1.27]$} \\
\hline & $p$-val & 0.531 & 0.812 & 0.917 & 0.985 & 0.941 & 0.754 & 0.534 & 0.796 & 0.893 & $0.003^{* *}$ & 0.177 & 0.197 & $0.01^{* *}$ & 0.207 & 0.230 & $0.003^{* *}$ & 0.196 & 0.205 \\
\hline & $R^{2}$ & 0.09 & 0.10 & 0.13 & 0.13 & 0.13 & 0.20 & 0.09 & 0.10 & 0.13 & 0.35 & 0.49 & 0.50 & 0.23 & 0.38 & 0.41 & 0.39 & 0.52 & 0.53 \\
\hline \multirow[t]{4}{*}{ PLS } & $\alpha(\%)$ & 0.003 & -0.025 & -0.042 & 0.063 & -0.005 & -0.041 & -0.010 & -0.055 & -0.086 & 0.321 & 0.174 & 0.164 & 0.314 & 0.166 & 0.151 & 0.633 & 0.348 & 0.329 \\
\hline & $t$-statistics & {$[0.01]$} & {$[-0.13]$} & {$[-0.23]$} & {$[0.44]$} & {$[-0.03]$} & {$[-0.28]$} & {$[-0.03]$} & {$[-0.14]$} & {$[-0.23]$} & {$[3.3]$} & {$[1.8]$} & {$[1.6]$} & {$[3.4]$} & {$[2.01]$} & {$[1.85]$} & {$[3.34]$} & [1.84] & {$[1.66]$} \\
\hline & $p$-val & 0.988 & 0.900 & 0.821 & 0.662 & 0.976 & 0.782 & 0.979 & 0.889 & 0.816 & $0.001^{* *}$ & 0.073 & 0.111 & $0.001^{* *}$ & $0.046^{*}$ & 0.065 & $0.001^{* *}$ & 0.067 & 0.099 \\
\hline & $R^{2}$ & 0.04 & 0.05 & 0.07 & 0.05 & 0.09 & 0.23 & 0.04 & 0.05 & 0.06 & 0.30 & 0.43 & 0.46 & 0.29 & 0.41 & 0.47 & 0.31 & 0.44 & 0.46 \\
\hline \multirow[t]{4}{*}{ PCR } & $\alpha(\%)$ & 0.376 & 0.168 & 0.131 & 0.020 & -0.046 & -0.083 & 0.697 & 0.302 & 0.231 & 0.359 & 0.171 & 0.160 & 0.298 & 0.187 & 0.178 & 0.708 & 0.343 & 0.321 \\
\hline & $t$-statistics & {$[1.43]$} & {$[0.71]$} & {$[0.59]$} & {$[0.07]$} & {$[-0.18]$} & {$[-0.32]$} & {$[1.37]$} & {$[0.66]$} & {$[0.54]$} & {$[3.23]$} & {$[1.83]$} & {$[1.77]$} & {$[3.88]$} & {$[2.34]$} & {$[2.19]$} & {$[3.24]$} & {$[1.87]$} & [1.81] \\
\hline & $p$-val & 0.153 & 0.478 & 0.554 & 0.943 & 0.854 & 0.747 & 0.172 & 0.508 & 0.589 & $0.001^{* *}$ & 0.068 & 0.078 & $0^{* *}$ & $0.02^{*}$ & $0.029^{*}$ & $0.001^{* *}$ & 0.063 & 0.072 \\
\hline & $R^{2}$ & 0.11 & 0.16 & 0.21 & 0.16 & 0.17 & 0.21 & 0.11 & 0.16 & 0.21 & 0.44 & 0.55 & 0.57 & 0.53 & 0.58 & 0.59 & 0.44 & 0.56 & 0.58 \\
\hline \multirow[t]{4}{*}{ LASSO } & $\alpha(\%)$ & 0.227 & 0.152 & 0.127 & 0.085 & 0.075 & 0.052 & 0.419 & 0.284 & 0.236 & 0.336 & 0.227 & 0.216 & 0.199 & 0.112 & 0.100 & 0.652 & 0.446 & 0.426 \\
\hline & $t$-statistics & {$[1.66]$} & {$[1]$} & {$[0.85]$} & {$[0.83]$} & {$[0.63]$} & {$[0.46]$} & {$[1.58]$} & {$[0.97]$} & {$[0.82]$} & {$[4.42]$} & {$[3.09]$} & {$[2.96]$} & {$[3.15]$} & {$[1.79]$} & {$[1.76]$} & {$[4.45]$} & {$[3.11]$} & {$[3.04]$} \\
\hline & $p$-val & 0.099 & 0.317 & 0.396 & 0.405 & 0.530 & 0.644 & 0.115 & 0.334 & 0.412 & $0^{* *}$ & $0.002^{* *}$ & $0.003^{* *}$ & $0.002^{* *}$ & 0.074 & 0.080 & $0^{* *}$ & $0.002^{* *}$ & $0.003^{* *}$ \\
\hline & $R^{2}$ & 0.11 & 0.12 & 0.19 & 0.08 & 0.09 & 0.17 & 0.10 & 0.12 & 0.19 & 0.21 & 0.30 & 0.32 & 0.12 & 0.24 & 0.30 & 0.21 & 0.30 & 0.33 \\
\hline \multirow[t]{4}{*}{ RIDGE } & $\alpha(\%)$ & 0.228 & 0.088 & 0.060 & 0.043 & -0.046 & -0.082 & 0.435 & 0.173 & 0.121 & 0.313 & 0.132 & 0.121 & 0.260 & 0.096 & 0.083 & 0.603 & 0.258 & 0.239 \\
\hline & $t$-statistics & {$[1.32]$} & {$[0.49]$} & {$[0.34]$} & {$[0.27]$} & {$[-0.27]$} & {$[-0.48]$} & {$[1.28]$} & {$[0.49]$} & {$[0.35]$} & [3.62] & {$[1.63]$} & {$[1.53]$} & {$[2.97]$} & {$[1.12]$} & {$[1.12]$} & {$[3.6]$} & {$[1.57]$} & {$[1.5]$} \\
\hline & $p$-val & $\begin{array}{l}1 . .24 \\
0.189\end{array}$ & 0.627 & 0.730 & 0.788 & 0.791 & 0.635 & 0.203 & 0.627 & 0.727 & $0^{* *}$ & 0.104 & 0.127 & $0.003^{* *}$ & 0.265 & 0.263 & $0^{* *}$ & 0.117 & 0.135 \\
\hline & $R^{2}$ & 0.12 & 0.16 & 0.22 & 0.09 & 0.11 & 0.20 & 0.12 & 0.16 & 0.21 & 0.36 & 0.54 & 0.57 & 0.32 & 0.49 & 0.54 & 0.37 & 0.54 & 0.57 \\
\hline \multirow[t]{4}{*}{ ENET } & $\alpha(\%)$ & 0.086 & -0.045 & -0.067 & 0.062 & 0.017 & -0.002 & 0.177 & -0.071 & -0.112 & 0.286 & 0.190 & 0.181 & 0.185 & 0.094 & 0.086 & 0.581 & 0.392 & 0.378 \\
\hline & $t$-statistics & {$[0.63]$} & {$[-0.33]$} & {$[-0.46]$} & {$[0.6]$} & {$[0.16]$} & {$[-0.02]$} & {$[0.67]$} & {$[-0.26]$} & {$[-0.39]$} & {$[3.33]$} & {$[2.37]$} & {$[2.25]$} & {$[2.93]$} & {$[1.72]$} & [1.64] & {$[3.39]$} & {$[2.47]$} & {$[2.37]$} \\
\hline & $p$-val & 0.528 & 0.742 & 0.643 & 0.551 & 0.873 & 0.982 & 0.504 & 0.795 & 0.700 & $0.001^{* *}$ & $0.018^{*}$ & $0.025^{*}$ & $0.004^{* *}$ & 0.087 & 0.102 & $0.001 * *$ & $0.014^{*}$ & $0.018^{*}$ \\
\hline & $R^{2}$ & 0.10 & 0.14 & 0.20 & 0.06 & 0.07 & 0.14 & 0.10 & 0.13 & 0.18 & 0.24 & 0.32 & 0.33 & 0.16 & 0.28 & 0.31 & 0.21 & 0.29 & 0.30 \\
\hline
\end{tabular}

Table B.9 reports the alphas, $t$-statistics, $p$-values and the $R^{2}$ of the regressions of the optimal portfolios generated by each mean-variance strategy (CMV, UMV, and MVATE), estimators (OLS, 3PRF, PLS, PCR, LASSO, Ridge, and ENet) using the FRED-MD as conditioning information on the Fama-French 3, 5, and $5+$ momentum factor models. "All Instruments" is the combination of Goyal and FRED-MD datasets with the Economic Policy Uncertainty (EPU) index and the Financial Stress Indicator (FSI). Goyal's variables comprises: b/m, dfr, dfy, infl, ltr, lty, ntis, svar, tms and tbl. FRED-MD is a large dataset containing 128 macroeconomic and financial variables. 
Table B.10: Alphas (Monthly \%) - 5 Industry Portfolios and 6 Portfolios Formed on Size and Book-to-Market - Goyal Variables

\begin{tabular}{|c|c|c|c|c|c|c|c|c|c|c|c|c|c|c|c|c|c|c|c|}
\hline & & \multicolumn{9}{|c|}{5 Industry Portfolios } & \multicolumn{9}{|c|}{6 Portfolios Formed on Size and Book-to-Market } \\
\hline & & \multicolumn{3}{|c|}{$\mathrm{CMV}$} & \multicolumn{3}{|c|}{ UMV } & \multicolumn{3}{|c|}{ MVATE } & \multicolumn{3}{|c|}{ CMV } & \multicolumn{3}{|c|}{ UMV } & \multicolumn{3}{|c|}{ MVATE } \\
\hline & & FF3 & FF5 & $\begin{array}{l}\text { FF5 + } \\
\text { Mom }\end{array}$ & FF3 & FF5 & $\begin{array}{l}\text { FF5 + } \\
\text { Mom }\end{array}$ & FF3 & FF5 & $\begin{array}{l}\text { FF5 + } \\
\text { Mom }\end{array}$ & FF3 & FF5 & $\begin{array}{l}\text { FF5 + } \\
\text { Mom }\end{array}$ & FF3 & FF5 & $\begin{array}{l}\text { FF5 + } \\
\text { Mom }\end{array}$ & FF3 & FF5 & $\begin{array}{l}\text { FF5 }+ \\
\text { Mom }\end{array}$ \\
\hline \multicolumn{20}{|c|}{ Z: Goyal } \\
\hline \multirow[t]{4}{*}{ OLS } & $\alpha(\%)$ & 0.202 & 0.138 & 0.109 & 0.197 & 0.122 & 0.073 & 0.394 & 0.277 & 0.222 & 0.220 & 0.125 & 0.107 & 0.205 & 0.117 & 0.101 & 0.406 & 0.238 & 0.206 \\
\hline & $t$-statistics & {$[2.43]$} & {$[1.53]$} & {$[1.26]$} & {$[2.04]$} & [1.07] & {$[0.7]$} & {$[2.43]$} & [1.59] & [1.31] & {$[3.25]$} & [1.86] & [1.64] & {$[3.45]$} & [1.91] & [1.68] & {$[3.17]$} & [1.86] & [1.64] \\
\hline & $p$-val & $0.016^{*}$ & 0.127 & 0.209 & $0.042^{*}$ & 0.284 & 0.484 & $0.016^{*}$ & 0.113 & 0.191 & $0.001^{* *}$ & 0.064 & 0.103 & $0.001^{* *}$ & 0.057 & 0.093 & $0.002^{* *}$ & 0.064 & 0.103 \\
\hline & $R^{2}$ & 0.09 & 0.12 & 0.22 & 0.14 & 0.17 & 0.35 & 0.09 & 0.11 & 0.20 & 0.11 & 0.21 & 0.27 & 0.09 & 0.21 & 0.29 & 0.13 & 0.21 & 0.27 \\
\hline \multirow[t]{4}{*}{ 3PRF } & $\alpha(\%)$ & 0.203 & 0.153 & 0.109 & 0.153 & 0.072 & 0.010 & 0.391 & 0.304 & 0.219 & 0.209 & 0.113 & 0.103 & 0.272 & 0.147 & 0.134 & 0.401 & 0.222 & 0.207 \\
\hline & $t$-statistics & {$[1.69]$} & {$[1.28]$} & {$[0.97]$} & {$[1.04]$} & {$[0.48]$} & {$[0.07]$} & [1.69] & {$[1.3]$} & {$[0.99]$} & {$[3.04]$} & {$[1.66]$} & {$[1.54]$} & {$[3.46]$} & {$[2.01]$} & [1.79] & {$[3.14]$} & [1.59] & {$[1.55]$} \\
\hline & $p$-val & 0.093 & 0.201 & 0.333 & 0.299 & 0.635 & 0.946 & 0.093 & 0.195 & 0.321 & $0.003^{* *}$ & 0.098 & 0.124 & $0.001^{* *}$ & $0.045^{*}$ & 0.075 & $0.002^{* *}$ & 0.113 & 0.122 \\
\hline & $R^{2}$ & 0.22 & 0.23 & 0.35 & 0.16 & 0.22 & 0.38 & 0.22 & 0.23 & 0.34 & 0.12 & 0.24 & 0.27 & 0.09 & 0.26 & 0.30 & 0.13 & 0.24 & 0.26 \\
\hline \multirow[t]{4}{*}{ PLS } & $\alpha(\%)$ & 0.352 & 0.236 & 0.185 & 0.528 & 0.408 & 0.289 & 0.676 & 0.462 & 0.362 & 0.310 & 0.204 & 0.190 & 0.363 & 0.219 & 0.193 & 0.595 & 0.399 & 0.373 \\
\hline & $t$-statistics & {$[2.04]$} & {$[1.3]$} & {$[1.02]$} & {$[2.62]$} & {$[1.53]$} & {$[1.25]$} & {$[2]$} & {$[1.28]$} & {$[1.02]$} & {$[3.35]$} & {$[2.06]$} & {$[2.23]$} & {$[3.64]$} & {$[2.58]$} & {$[2.21]$} & {$[3.31]$} & {$[2.06]$} & {$[2.24]$} \\
\hline & $p$-val & $0.042^{*}$ & 0.196 & 0.307 & $0.009^{* *}$ & 0.128 & 0.214 & $0.046^{*}$ & 0.202 & 0.308 & $0.001^{* *}$ & $0.04^{*}$ & $0.026^{*}$ & $0^{* *}$ & $0.01^{*}$ & $0.028^{*}$ & $0.001^{* *}$ & $0.04^{*}$ & $0.026^{*}$ \\
\hline & $R^{2}$ & 0.13 & 0.16 & 0.23 & 0.15 & 0.21 & 0.39 & 0.13 & 0.15 & 0.22 & 0.34 & 0.41 & 0.43 & 0.25 & 0.34 & 0.40 & 0.34 & 0.41 & 0.43 \\
\hline \multirow[t]{4}{*}{ PCR } & $\alpha(\%)$ & 0.184 & -0.141 & -0.198 & 0.164 & -0.126 & -0.164 & 0.329 & -0.285 & -0.396 & 0.405 & 0.245 & 0.236 & 0.401 & 0.233 & 0.224 & 0.781 & 0.480 & 0.462 \\
\hline & $t$-statistics & {$[1.02]$} & {$[-0.81]$} & {$[-1.08]$} & {$[0.86]$} & {$[-0.67]$} & {$[-0.84]$} & [0.95] & {$[-0.84]$} & {$[-1.11]$} & [4.68] & {$[3.12]$} & {$[2.97]$} & {$[4.42]$} & {$[2.83]$} & {$[2.75]$} & {$[4.7]$} & {$[3.24]$} & {$[3]$} \\
\hline & $p$-val & 0.307 & 0.420 & 0.280 & 0.390 & 0.506 & 0.400 & 0.343 & 0.402 & 0.267 & $0^{* *}$ & $0.002^{* *}$ & $0.003^{* *}$ & $0^{* *}$ & $0.005^{* *}$ & $0.006^{* *}$ & $0^{* *}$ & $0.001^{* *}$ & $0.003^{* *}$ \\
\hline & $R^{2}$ & 0.11 & 0.22 & 0.30 & 0.12 & 0.22 & 0.26 & 0.11 & 0.21 & 0.29 & 0.41 & 0.53 & 0.54 & 0.39 & 0.53 & 0.54 & 0.42 & 0.54 & 0.55 \\
\hline \multirow[t]{4}{*}{ LASSO } & $\alpha(\%)$ & 0.341 & 0.300 & 0.260 & 0.241 & 0.178 & 0.139 & 0.659 & 0.588 & 0.510 & 0.186 & 0.107 & 0.092 & 0.161 & 0.080 & 0.069 & 0.356 & 0.206 & 0.179 \\
\hline & $t$-statistics & {$[3.43]$} & {$[2.76]$} & {$[2.73]$} & {$[2.72]$} & {$[2.02]$} & {$[1.48]$} & {$[3.41]$} & {$[2.78]$} & {$[2.81]$} & {$[2.6]$} & {$[1.47]$} & {$[1.23]$} & {$[3.05]$} & {$[1.63]$} & {$[1.3]$} & {$[2.63]$} & {$[1.5]$} & {$[1.25]$} \\
\hline & $p$-val & $0.001^{* *}$ & $0.006^{* *}$ & $0.007^{* *}$ & $0.007^{* *}$ & $0.044^{*}$ & 0.140 & $0.001^{* *}$ & $0.006^{* *}$ & $0.005^{* *}$ & $0.01^{* *}$ & 0.142 & 0.220 & $0.003^{* *}$ & 0.104 & 0.196 & $0.009^{* *}$ & 0.134 & 0.211 \\
\hline & $R^{2}$ & 0.11 & 0.11 & 0.26 & 0.08 & 0.10 & 0.25 & 0.10 & 0.11 & 0.26 & 0.24 & 0.29 & 0.33 & 0.22 & 0.32 & 0.36 & 0.26 & 0.31 & 0.34 \\
\hline \multirow[t]{4}{*}{ RIDGE } & $\alpha(\%)$ & 0.231 & 0.154 & 0.118 & 0.198 & 0.116 & 0.078 & 0.447 & 0.311 & 0.241 & 0.223 & 0.069 & 0.058 & 0.230 & 0.100 & 0.086 & 0.434 & 0.138 & 0.117 \\
\hline & $t$-statistics & {$[2.2]$} & [1.58] & [1.17] & {$[2.29]$} & [1.28] & {$[0.94]$} & {$[2.17]$} & {$[1.66]$} & {$[1.23]$} & {$[2.42]$} & {$[0.86]$} & {$[0.72]$} & {$[3.18]$} & [1.48] & [1.23] & {$[2.47]$} & {$[0.9]$} & {$[0.76]$} \\
\hline & $p$-val & $0.029^{*}$ & 0.114 & 0.245 & $0.023^{*}$ & 0.203 & 0.347 & $0.031^{*}$ & 0.099 & 0.218 & $0.016^{*}$ & 0.390 & 0.475 & $0.002^{* *}$ & 0.140 & 0.219 & $0.014^{*}$ & 0.369 & 0.448 \\
\hline & $R^{2}$ & 0.13 & 0.15 & 0.24 & 0.16 & 0.21 & 0.34 & 0.12 & 0.14 & 0.23 & 0.26 & 0.40 & 0.42 & 0.13 & 0.31 & 0.35 & 0.27 & 0.42 & 0.43 \\
\hline \multirow[t]{4}{*}{ ENET } & $\alpha(\%)$ & 0.296 & 0.245 & 0.204 & 0.225 & 0.120 & 0.084 & 0.594 & 0.500 & 0.421 & 0.264 & 0.163 & 0.152 & 0.229 & 0.146 & 0.131 & 0.525 & 0.329 & 0.311 \\
\hline & $t$-statistics & {$[2.76]$} & {$[2.2]$} & {$[1.83]$} & {$[2.44]$} & {$[1.4]$} & {$[0.93]$} & {$[2.7]$} & {$[2.31]$} & {$[1.94]$} & {$[3.92]$} & {$[2.6]$} & {$[2.53]$} & {$[4.14]$} & {$[3.08]$} & {$[2.53]$} & {$[4.04]$} & {$[2.73]$} & {$[2.69]$} \\
\hline & $p$-val & $0.006^{* *}$ & $0.029^{*}$ & 0.068 & $0.015^{*}$ & 0.162 & 0.352 & $0.007^{* *}$ & $0.021^{*}$ & 0.054 & $0^{* *}$ & $0.01^{* *}$ & $0.012^{*}$ & $0^{* *}$ & $0.002^{* *}$ & $0.012^{*}$ & $0^{* *}$ & $0.007^{* *}$ & $0.008^{* *}$ \\
\hline & $R^{2}$ & 0.13 & 0.14 & 0.26 & 0.06 & 0.11 & 0.23 & 0.13 & 0.13 & 0.25 & 0.21 & 0.30 & 0.32 & 0.15 & 0.26 & 0.33 & 0.22 & 0.31 & 0.32 \\
\hline
\end{tabular}

Table B.10 reports the alphas, $t$-statistics, $p$-values and the $R^{2}$ of the regressions of the optimal portfolios generated by each mean-variance strategy (CMV, UMV, and MVATE), estimators (OLS, 3PRF, PLS, PCR, LASSO, Ridge, and ENet) using Goyal's variables as conditioning information on the Fama-French 3, 5, and $5+$ momentum factor models. Goyal's variables comprises: b/m, dfr, dfy, infl, ltr, lty, ntis, svar, tms and tbl. 


\section{B.6 Financial Metrics}

Table B.11: Turnover and Financial Metrics (Monthly \%)

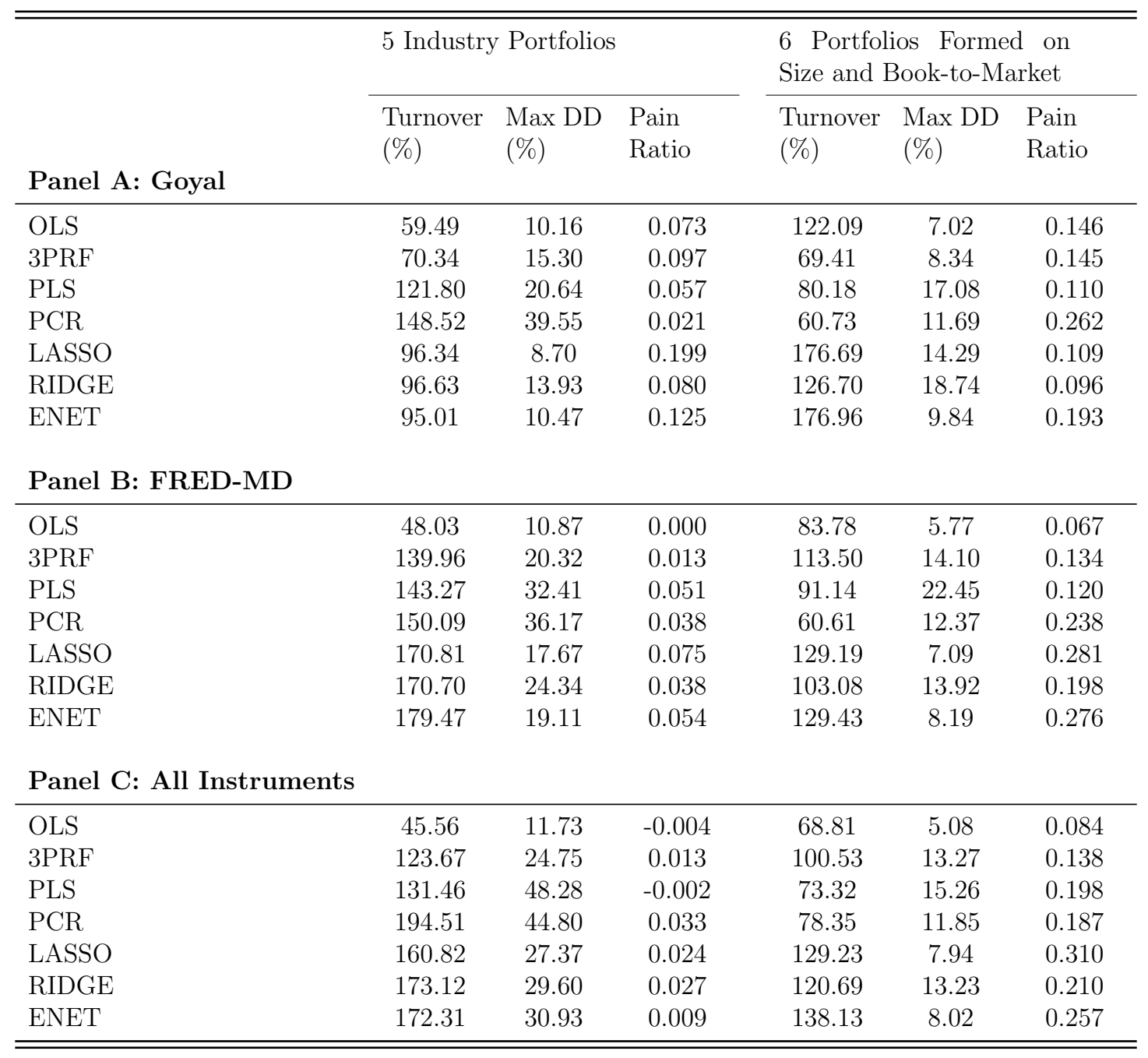

Table B.11 reports several standard financial metrics for the 5 Industry portfolios and 6 portfolios formed on Size/BTM to evaluate portfolios by estimator for the CMV framework. The Turnover is computed following equation (38). MaxDD is maximum drawdown as presented in equation (39). Pain Ratio is the standard metric, as shown in equation (37). Panel A reports the Sharpe ratios generated when the variables from Goyal's website are used as $\mathbf{Z}$. Goyal variables comprises: $b / m$, dfr, dfy, infl, ltr, lty, ntis, svar, tms and tbl. Panel B presents the Sharpe ratios obtained using the FRED-MD variables. The FRED-MD is a large dataset containing 128 macroeconomic and financial variables. Finally, panel C shows the Sharpe rations when all variables are used as conditioning information. "All Instruments" is the combination of Goyal and FRED-MD datasets with the Economic Policy Uncertainty (EPU) index and the Financial Stress Indicator (FSI). 


\section{Appendix - Data}

Table C.1: FRED-MD

\begin{tabular}{|c|c|c|c|c|c|c|c|c|c|}
\hline \multirow[b]{2}{*}{ Fred Code } & \multirow[b]{2}{*}{ tcode } & \multirow[b]{2}{*}{ Group } & \multirow[b]{2}{*}{ Description } & \multicolumn{2}{|c|}{ Full Sample } & \multicolumn{2}{|c|}{ In-Sample } & \multicolumn{2}{|c|}{ Out-of-Sample } \\
\hline & & & & Mean & sd & Mean & sd & Mean & sd \\
\hline RPI & 5 & Output and income & Real Personal Income & 0.0020 & 0.0070 & 0.0020 & 0.0090 & 0.0020 & 0.0070 \\
\hline W875RX1 & 5 & Output and income & Real personal income ex transfer receipts & 0.0020 & 0.0070 & 0.0020 & 0.0110 & 0.0020 & 0.0070 \\
\hline INDPRO & 5 & Output and income & IP Index & 0.0020 & 0.0060 & 0.0040 & 0.0040 & 0.0010 & 0.0070 \\
\hline IPFPNSS & 5 & Output and income & IP: Final Products and Nonindustrial Supplies & 0.0010 & 0.0060 & 0.0030 & 0.0040 & 0.0010 & 0.0060 \\
\hline IPFINAL & 5 & Output and income & IP: Final Products (Market Group) & 0.0010 & 0.0070 & 0.0030 & 0.0050 & 0.0010 & 0.0070 \\
\hline IPCONGD & 5 & Output and income & IP: Consumer Goods & 0.0010 & 0.0070 & 0.0030 & 0.0060 & 0.0000 & 0.0070 \\
\hline IPDCONGD & 5 & Output and income & IP: Durable Consumer Goods & 0.0020 & 0.0190 & 0.0060 & 0.0120 & 0.0010 & 0.0200 \\
\hline IPNCONGD & 5 & Output and income & IP: Nondurable Consumer Goods & 0.0000 & 0.0070 & 0.0020 & 0.0050 & 0.0000 & 0.0070 \\
\hline IPBUSEQ & 5 & Output and income & IP: Business Equipment & 0.0030 & 0.0120 & 0.0060 & 0.0070 & 0.0020 & 0.0130 \\
\hline IPMAT & 5 & Output and income & IP: Materials & 0.0020 & 0.0080 & 0.0040 & 0.0040 & 0.0020 & 0.0090 \\
\hline IPDMAT & 5 & Output and income & IP: Durable Materials & 0.0040 & 0.0110 & 0.0070 & 0.0070 & 0.0030 & 0.0110 \\
\hline IPNMAT & 5 & Output and income & IP: Nondurable Materials & 0.0000 & 0.0110 & 0.0010 & 0.0070 & 0.0000 & 0.0120 \\
\hline IPMANSICS & 5 & Output and income & IP: Manufacturing (SIC) & 0.0020 & 0.0070 & 0.0040 & 0.0050 & 0.0010 & 0.0070 \\
\hline IPB51222s & 5 & Output and income & IP: Residential Utilities & 0.0010 & 0.0400 & 0.0030 & 0.0330 & 0.0010 & 0.0410 \\
\hline IPFUELS & 5 & Output and income & IP: Fuels & 0.0010 & 0.0190 & 0.0010 & 0.0160 & 0.0010 & 0.0190 \\
\hline CUMFNS & 2 & Output and income & Capacity Utilization: Manufacturing & -0.0090 & 0.5000 & 0.0780 & 0.3790 & -0.0240 & 0.5170 \\
\hline HWI & 2 & Labor market & Help-Wanted Index for United States & 10.9680 & 220.7630 & 33.7610 & 123.4940 & 6.9960 & 233.5480 \\
\hline HWIURATIO & 2 & Labor market & Ratio of Help Wanted/No. Unemployed & 0.0020 & 0.0330 & 0.0060 & 0.0180 & 0.0010 & 0.0350 \\
\hline CLF16OV & 5 & Labor market & Civilian Labor Force & 0.0010 & 0.0020 & 0.0010 & 0.0020 & 0.0010 & 0.0020 \\
\hline CE16OV & 5 & Labor market & Civilian Employment & 0.0010 & 0.0020 & 0.0010 & 0.0020 & 0.0010 & 0.0020 \\
\hline UNRATE & 2 & Labor market & Civilian Unemployment Rate & -0.0110 & 0.1560 & -0.0390 & 0.1320 & -0.0060 & 0.1590 \\
\hline UEMPMEAN & 2 & Labor market & Average Duration of Unemployment (Weeks) & 0.0230 & 0.6950 & -0.0090 & 0.4930 & 0.0280 & 0.7250 \\
\hline UEMPLT5 & 5 & Labor market & Civilians Unemployed - Less Than 5 Weeks & -0.0010 & 0.0600 & -0.0050 & 0.0520 & -0.0010 & 0.0620 \\
\hline UEMP5TO14 & 5 & Labor market & Civilians Unemployed for 43599 Weeks & -0.0010 & 0.0520 & -0.0040 & 0.0500 & -0.0010 & 0.0520 \\
\hline UEMP15OV & 5 & Labor market & Civilians Unemployed - 15 Weeks \& Over & -0.0010 & 0.0450 & -0.0070 & 0.0460 & 0.0000 & 0.0440 \\
\hline UEMP15T26 & 5 & Labor market & Civilians Unemployed for 15-26 Weeks & -0.0020 & 0.0710 & -0.0060 & 0.0820 & -0.0010 & 0.0700 \\
\hline UEMP27OV & 5 & Labor market & Civilians Unemployed for 27 Weeks and Over & 0.0000 & 0.0570 & -0.0070 & 0.0500 & 0.0010 & 0.0590 \\
\hline CLAIMSx & 5 & Labor market & Initial Claims & -0.0020 & 0.0410 & -0.0040 & 0.0380 & -0.0020 & 0.0420 \\
\hline PAYEMS & 5 & Labor market & All Employees: Total Nonfarm & 0.0010 & 0.0020 & 0.0020 & 0.0010 & 0.0010 & 0.0020 \\
\hline USGOOD & 5 & Labor market & All Employees: Goods-Producing Industries & 0.0000 & 0.0040 & 0.0010 & 0.0020 & -0.0010 & 0.0040 \\
\hline CES1021000001 & 5 & Labor market & All Employees: Mining and Logging: Mining & 0.0000 & 0.0090 & -0.0030 & 0.0060 & 0.0010 & 0.0090 \\
\hline USCONS & 5 & Labor market & All Employees: Construction & 0.0010 & 0.0060 & 0.0030 & 0.0050 & 0.0010 & 0.0060 \\
\hline MANEMP & 5 & Labor market & All Employees: Manufacturing & -0.0010 & 0.0030 & 0.0010 & 0.0010 & -0.0010 & 0.0040 \\
\hline DMANEMP & 5 & Labor market & All Employees: Durable goods & -0.0010 & 0.0040 & 0.0010 & 0.0020 & -0.0010 & 0.0050 \\
\hline NDMANEMP & 5 & Labor market & All Employees: Nondurable goods & -0.0010 & 0.0020 & 0.0000 & 0.0010 & -0.0010 & 0.0030 \\
\hline SRVPRD & 5 & Labor market & All Employees: Service-Providing Industries & 0.0010 & 0.0010 & 0.0020 & 0.0010 & 0.0010 & 0.0010 \\
\hline USTPU & 5 & Labor market & All Employees: Trade, Transportation \& Utilities & 0.0010 & 0.0020 & 0.0020 & 0.0020 & 0.0010 & 0.0020 \\
\hline USWTRADE & 5 & Labor market & All Employees: Wholesale Trade & 0.0000 & 0.0020 & 0.0010 & 0.0020 & 0.0000 & 0.0020 \\
\hline USTRADE & 5 & Labor market & All Employees: Retail Trade & 0.0010 & 0.0020 & 0.0020 & 0.0020 & 0.0000 & 0.0020 \\
\hline USFIRE & 5 & Labor market & All Employees: Financial Activities & 0.0010 & 0.0020 & 0.0010 & 0.0020 & 0.0010 & 0.0020 \\
\hline USGOVT & 5 & Labor market & All Employees: Government & 0.0010 & 0.0020 & 0.0010 & 0.0010 & 0.0010 & 0.0030 \\
\hline CES0600000007 & 1 & Labor market & Avg Weekly Hours : Goods-Producing & 40.6080 & 0.5840 & 40.7170 & 0.3270 & 40.5890 & 0.6170 \\
\hline AWOTMAN & 2 & Labor market & Avg Weekly Overtime Hours : Manufacturing & 0.0020 & 0.1130 & 0.0150 & 0.1560 & -0.0010 & 0.1040 \\
\hline AWHMAN & 1 & Labor market & Avg Weekly Hours : Manufacturing & 41.1990 & 0.5940 & 41.2390 & 0.3630 & 41.1920 & 0.6250 \\
\hline CES0600000008 & 6 & Labor market & Avg Hourly Earnings : Goods-Producing & 0.0000 & 0.0030 & 0.0000 & 0.0020 & 0.0000 & 0.0030 \\
\hline CES2000000008 & 6 & Labor market & Avg Hourly Earnings : Construction & 0.0000 & 0.0060 & 0.0000 & 0.0060 & 0.0000 & 0.0050 \\
\hline CES3000000008 & 6 & Labor market & Avg Hourly Earnings : Manufacturing & 0.0000 & 0.0030 & 0.0000 & 0.0020 & 0.0000 & 0.0030 \\
\hline HOUST & 4 & Housing & Housing Starts: Total New Privately Owned & 7.1220 & 0.3740 & 7.1890 & 0.0920 & 7.1110 & 0.4020 \\
\hline HOUSTNE & 4 & Housing & Housing Starts, Northeast & 4.8070 & 0.3400 & 4.8340 & 0.1200 & 4.8020 & 0.3640 \\
\hline HOUSTMW & 4 & Housing & Housing Starts, Midwest & 5.4140 & 0.4800 & 5.6980 & 0.1040 & 5.3640 & 0.5020 \\
\hline HOUSTS & 4 & Housing & Housing Starts, South & 6.3780 & 0.3470 & 6.3630 & 0.1180 & 6.3800 & 0.3730 \\
\hline HOUSTW & 4 & Housing & Housing Starts, West & 5.6850 & 0.4370 & 5.7590 & 0.1180 & 5.6720 & 0.4700 \\
\hline PERMIT & 4 & Housing & New Private Housing Permits (SAAR) & 7.1370 & 0.3670 & 7.1320 & 0.1050 & 7.1380 & 0.3950 \\
\hline PERMITNE & 4 & Housing & New Private Housing Permits, Northeast (SAAR) & 4.8700 & 0.3340 & 4.8650 & 0.1040 & 4.8710 & 0.3600 \\
\hline PERMITMW & 4 & Housing & New Private Housing Permits, Midwest (SAAR) & 5.4310 & 0.4410 & 5.6480 & 0.0880 & 5.3930 & 0.4660 \\
\hline PERMITS & 4 & Housing & New Private Housing Permits, South (SAAR) & 6.3790 & 0.3400 & 6.2720 & 0.1320 & 6.3980 & 0.3610 \\
\hline PERMITW & 4 & Housing & New Private Housing Permits, West (SAAR) & 5.7120 & 0.4350 & 5.7260 & 0.1240 & 5.7090 & 0.4680 \\
\hline DPCERA3M086SBEA & 5 & Consumption, orders, and inventories & Real Personal Consumption Expenditures & 0.0020 & 0.0030 & 0.0030 & 0.0030 & 0.0020 & 0.0030 \\
\hline CMRMTSPLx & 5 & Consumption, orders, and inventories & Real Manu. and Trade Industries Sales & 0.0020 & 0.0080 & 0.0040 & 0.0080 & 0.0020 & 0.0080 \\
\hline RETAILx & 5 & Consumption, orders, and inventories & Retail and Food Services Sales & 0.0040 & 0.0090 & 0.0050 & 0.0080 & 0.0030 & 0.0100 \\
\hline ACOGNO & 5 & Consumption, orders, and inventories & New Orders for Consumer Goods & 0.0030 & 0.0190 & 0.0050 & 0.0150 & 0.0020 & 0.0190 \\
\hline AMDMNOx & 5 & Consumption, orders, and inventories & New Orders for Durable Goods & 0.0020 & 0.0410 & 0.0080 & 0.0250 & 0.0020 & 0.0440 \\
\hline ANDENOx & 5 & Consumption, orders, and inventories & New Orders for Nondefense Capital Goods & 0.0020 & 0.0810 & 0.0100 & 0.0480 & 0.0010 & 0.0850 \\
\hline AMDMUOx & 5 & Consumption, orders, and inventories & Unfilled Orders for Durable Goods & 0.0030 & 0.0100 & -0.0010 & 0.0050 & 0.0040 & 0.0100 \\
\hline BUSINVx & 5 & Consumption, orders, and inventories & Total Business Inventories & 0.0030 & 0.0050 & 0.0040 & 0.0030 & 0.0020 & 0.0050 \\
\hline ISRATIOx & 2 & Consumption, orders, and inventories & Total Business: Inventories to Sales Ratio & -0.0010 & 0.0140 & -0.0020 & 0.0130 & 0.0000 & 0.0150 \\
\hline UMCSENTx & 2 & Consumption, orders, and inventories & Consumer Sentiment Index & 0.0870 & 3.8890 & 0.4830 & 3.5940 & 0.0190 & 3.9410 \\
\hline
\end{tabular}

(Continued) 
Table C.1: FRED-MD (Continued)

\begin{tabular}{|c|c|c|c|c|c|c|c|c|c|}
\hline \multirow[b]{2}{*}{ Fred Code } & \multirow[b]{2}{*}{ tcode } & \multirow[b]{2}{*}{ Group } & \multirow[b]{2}{*}{ Description } & \multicolumn{2}{|c|}{ Full Sample } & \multicolumn{2}{|c|}{ In-Sample } & \multicolumn{2}{|c|}{ Out-of-Sample } \\
\hline & & & & Mean & sd & Mean & sd & Mean & sd \\
\hline M1SL & 6 & Money and credit & M1 Money Stock & 0.000 & 0.012 & 0.000 & 0.003 & 0.000 & 0.013 \\
\hline M2SL & 6 & Money and credit & M2 Money Stock & 0.000 & 0.004 & 0.000 & 0.003 & 0.000 & 0.004 \\
\hline M2REAL & 5 & Money and credit & Real M2 Money Stock & 0.003 & 0.005 & -0.001 & 0.003 & 0.003 & 0.005 \\
\hline AMBSL & 6 & Money and credit & St. Louis Adjusted Monetary Base & 0.000 & 0.025 & 0.000 & 0.003 & 0.000 & 0.027 \\
\hline TOTRESNS & 6 & Money and credit & Total Reserves of Depository Institutions & 0.000 & 0.089 & 0.001 & 0.037 & 0.000 & 0.096 \\
\hline NONBORRES & 7 & Money and credit & Reserves Of Depository Institutions & 0.000 & 1.618 & 0.001 & 0.036 & 0.000 & 1.754 \\
\hline BUSLOANS & 6 & Money and credit & Commercial and Industrial Loans & 0.000 & 0.006 & 0.000 & 0.005 & 0.000 & 0.007 \\
\hline REALLN & 6 & Money and credit & Real Estate Loans at All Commercial Banks & 0.000 & 0.007 & 0.000 & 0.003 & 0.000 & 0.007 \\
\hline NONREVSL & 6 & Money and credit & Total Nonrevolving Credit & 0.000 & 0.008 & 0.000 & 0.007 & 0.000 & 0.008 \\
\hline CONSPI & 2 & Money and credit & Nonrevolving Consumer Credit to Personal Income & 0.000 & 0.001 & 0.000 & 0.001 & 0.000 & 0.001 \\
\hline MZMSL & 6 & Money and credit & MZM Money Stock & 0.000 & 0.005 & 0.000 & 0.004 & 0.000 & 0.005 \\
\hline DTCOLNVHFNM & 6 & Money and credit & Consumer Motor Vehicle Loans Outstanding & 0.000 & 0.029 & 0.000 & 0.024 & 0.000 & 0.030 \\
\hline DTCTHFNM & 6 & Money and credit & Total Consumer Loans and Leases Outstanding & 0.000 & 0.028 & 0.000 & 0.011 & 0.000 & 0.030 \\
\hline INVEST & 6 & Money and credit & Securities in Bank Credit at All Commercial Banks & 0.000 & 0.012 & 0.000 & 0.012 & 0.000 & 0.012 \\
\hline FEDFUNDS & 2 & Interest and exchange rates & Effective Federal Funds Rate & -0.009 & 0.165 & 0.033 & 0.178 & -0.016 & 0.162 \\
\hline CP3Mx & 2 & Interest and exchange rates & 3-Month AA Financial Commercial Paper Rate & -0.008 & 0.194 & 0.033 & 0.207 & -0.016 & 0.192 \\
\hline TB3MS & 2 & Interest and exchange rates & 3-Month Treasury Bill & -0.008 & 0.177 & 0.028 & 0.180 & -0.014 & 0.176 \\
\hline TB6MS & 2 & Interest and exchange rates & 6-Month Treasury Bill & -0.008 & 0.175 & 0.026 & 0.209 & -0.014 & 0.168 \\
\hline GS1 & 2 & Interest and exchange rates & 1-Year Treasury Rate & -0.008 & 0.195 & 0.022 & 0.260 & -0.014 & 0.181 \\
\hline GS5 & 2 & Interest and exchange rates & 5-Year Treasury Rate & -0.014 & 0.236 & -0.023 & 0.279 & -0.013 & 0.228 \\
\hline GS10 & 2 & Interest and exchange rates & 10-Year Treasury Rate & -0.016 & 0.220 & -0.035 & 0.239 & -0.013 & 0.217 \\
\hline $\mathrm{AAA}$ & 2 & Interest and exchange rates & Moody's Seasoned Aaa Corporate Bond & -0.015 & 0.177 & -0.032 & 0.179 & -0.013 & 0.176 \\
\hline BAA & 2 & Interest and exchange rates & Moody's Seasoned Baa Corporate Bond & -0.016 & 0.201 & -0.038 & 0.191 & -0.012 & 0.203 \\
\hline COMPAPFFx & 1 & Interest and exchange rates & 3-Month Commercial Paper Minus & 0.144 & 0.242 & 0.255 & 0.229 & 0.125 & 0.239 \\
\hline TB3SMFFM & 1 & Interest and exchange rates & 3-Month Treasury C Minus & -0.196 & 0.259 & -0.099 & 0.210 & -0.213 & 0.264 \\
\hline TB6SMFFM & 1 & Interest and exchange rates & 6-Month Treasury C Minus & -0.090 & 0.307 & 0.081 & 0.341 & -0.120 & 0.292 \\
\hline T1YFFM & 1 & Interest and exchange rates & 1-Year Treasury C Minus & 0.140 & 0.409 & 0.514 & 0.512 & 0.075 & 0.351 \\
\hline T5YFFM & 1 & Interest and exchange rates & 5-Year Treasury C Minus & 1.110 & 0.965 & 1.939 & 0.961 & 0.965 & 0.891 \\
\hline T10YFFM & 1 & Interest and exchange rates & 10-Year Treasury C Minus & 1.701 & 1.251 & 2.462 & 1.143 & 1.568 & 1.223 \\
\hline AAAFFM & 1 & Interest and exchange rates & Moody's Aaa Corporate Bond Minus & 3.167 & 1.451 & 3.555 & 1.195 & 3.100 & 1.483 \\
\hline BAAFFM & 1 & Interest and exchange rates & Moody's Baa Corporate Bond Minus & 4.115 & 1.658 & 4.250 & 1.256 & 4.092 & 1.720 \\
\hline TWEXMMTH & 5 & Interest and exchange rates & Trade Weighted U.S. Dollar Index: Major Currencies & 0.000 & 0.016 & -0.001 & 0.016 & 0.000 & 0.017 \\
\hline EXSZUSx & 5 & Interest and exchange rates & Switzerland / U.S. Foreign Exchange Rate & -0.001 & 0.025 & -0.005 & 0.031 & -0.001 & 0.024 \\
\hline EXJPUSx & 5 & Interest and exchange rates & Japan / U.S. Foreign Exchange Rate & 0.000 & 0.026 & -0.005 & 0.030 & 0.000 & 0.025 \\
\hline EXUSUKx & 5 & Interest and exchange rates & U.S. / U.K. Foreign Exchange Rate & -0.001 & 0.022 & -0.003 & 0.029 & -0.001 & 0.021 \\
\hline EXCAUSx & 5 & Interest and exchange rates & Canada / U.S. Foreign Exchange Rate & 0.000 & 0.018 & 0.003 & 0.011 & 0.000 & 0.018 \\
\hline WPSFD49207 & 6 & Prices & PPI: Finished Goods & 0.000 & 0.008 & 0.000 & 0.003 & 0.000 & 0.008 \\
\hline WPSFD49502 & 6 & Prices & PPI: Finished Consumer Goods & 0.000 & 0.010 & 0.000 & 0.004 & 0.000 & 0.011 \\
\hline WPSID61 & 6 & Prices & PPI: Intermediate Materials & 0.000 & 0.009 & 0.000 & 0.003 & 0.000 & 0.009 \\
\hline WPSID62 & 6 & Prices & PPI: Crude Materials & 0.000 & 0.051 & 0.000 & 0.020 & 0.000 & 0.055 \\
\hline OILPRICEx & 6 & Prices & Crude Oil, Spliced WTI and Cushing & 0.000 & 0.099 & 0.001 & 0.062 & 0.000 & 0.105 \\
\hline PPICMM & 6 & Prices & PPI: Metals and Metal Products: & 0.000 & 0.039 & -0.001 & 0.022 & 0.000 & 0.042 \\
\hline CPIAUCSL & 6 & Prices & CPI : All Items & 0.000 & 0.003 & 0.000 & 0.001 & 0.000 & 0.003 \\
\hline CPIAPPSL & 6 & Prices & CPI : Apparel & 0.000 & 0.006 & 0.000 & 0.006 & 0.000 & 0.006 \\
\hline CPITRNSL & 6 & Prices & CPI : Transportation & 0.000 & 0.015 & 0.000 & 0.005 & 0.000 & 0.016 \\
\hline CPIMEDSL & 6 & Prices & CPI : Medical Care & 0.000 & 0.002 & 0.000 & 0.001 & 0.000 & 0.002 \\
\hline CUSR0000SAC & 6 & Prices & CPI : Commodities & 0.000 & 0.007 & 0.000 & 0.003 & 0.000 & 0.007 \\
\hline CUSR0000SAD & 6 & Prices & CPI : Durables & 0.000 & 0.002 & 0.000 & 0.002 & 0.000 & 0.002 \\
\hline CUSR0000SAS & 6 & Prices & CPI : Services & 0.000 & 0.001 & 0.000 & 0.001 & 0.000 & 0.001 \\
\hline CPIULFSL & 6 & Prices & CPI : All Items Less Food & 0.000 & 0.003 & 0.000 & 0.001 & 0.000 & 0.003 \\
\hline CUSR0000SA0L2 & 6 & Prices & CPI : All Items Less Shelter & 0.000 & 0.004 & 0.000 & 0.002 & 0.000 & 0.004 \\
\hline CUSR0000SA0L5 & 6 & Prices & CPI : All Items Less Medical Care & 0.000 & 0.003 & 0.000 & 0.001 & 0.000 & 0.003 \\
\hline PCEPI & 6 & Prices & Personal Cons. Expend.: Chain Index & 0.000 & 0.002 & 0.000 & 0.001 & 0.000 & 0.002 \\
\hline DDURRG3M086SBEA & 6 & Prices & Personal Cons. Exp: Durable goods & 0.000 & 0.003 & 0.000 & 0.003 & 0.000 & 0.003 \\
\hline DNDGRG3M086SBEA & 6 & Prices & Personal Cons. Exp: Nondurable goods & 0.000 & 0.008 & 0.000 & 0.003 & 0.000 & 0.008 \\
\hline DSERRG3M086SBEA & 6 & Prices & Personal Cons. Exp: Services & 0.000 & 0.002 & 0.000 & 0.001 & 0.000 & 0.002 \\
\hline S\&P 500 & 5 & Stock market & S\&P's Common Stock Price Index: Composite & 0.006 & 0.035 & 0.009 & 0.017 & 0.006 & 0.038 \\
\hline $\mathrm{S} \& \mathrm{P}$ div yield & 2 & Stock market & S\&P's Composite Common Stock: Dividend Yield & -0.004 & 0.077 & -0.016 & 0.046 & -0.002 & 0.081 \\
\hline S\&P PE ratio & 5 & Stock market & S\&P's Composite Common Stock: Price-Earnings Ratio & 0.001 & 0.053 & -0.004 & 0.018 & 0.001 & 0.057 \\
\hline VXOCLSx & 1 & Stock market & VXO & 19.650 & 8.723 & 13.341 & 1.497 & 20.750 & 8.992 \\
\hline
\end{tabular}

As in McCracken and $\mathrm{Ng}(2016)$ we transform the variables following the code presented in column 'tcode'. The transformation for a series $x$ are: (1) no transformation; (2) $\Delta x_{t} ;(3) \Delta^{2} x_{t}$; (4) $\log \left(x_{t}\right),(5) \Delta \log \left(x_{t}\right)$, (6) $\Delta^{2} \log \left(x_{t}\right)$, and (7) $\Delta\left(x_{t} / x_{t-1}-1\right)$. The column 'gsi' and 'gsi:description' present the comparable series in Global Insight. 
Table C.2: Results for the 5 Industry Portfolios

\begin{tabular}{|c|c|c|c|c|c|c|c|c|c|c|c|c|c|c|}
\hline \multirow[t]{3}{*}{ A: } & \multicolumn{14}{|l|}{ CMV } \\
\hline & \multirow[b]{2}{*}{ Estimator } & \multirow[b]{2}{*}{$\hat{\mu}$} & \multirow[b]{2}{*}{$\hat{\sigma}$} & \multirow[b]{2}{*}{$\hat{\rho}_{1}$} & \multirow[b]{2}{*}{$\hat{\rho}_{2}$} & \multicolumn{2}{|c|}{ Monthly } & \multicolumn{7}{|c|}{ Annual } \\
\hline & & & & & & $S R$ & se & $S R$ & se & $t$ & $p$-val & $s e(H A C)$ & $t(H A C)$ & $p(H A C)$ \\
\hline & \multicolumn{14}{|l|}{ Goyal } \\
\hline & OLS & 0.002 & 0.015 & -0.108 & 0.018 & 0.133 & 0.062 & 0.462 & 0.214 & 2.157 & 0.032 & 0.038 & 12.087 & 0.000 \\
\hline & $3 \mathrm{PRF}$ & 0.003 & 0.021 & -0.106 & 0.089 & 0.125 & 0.062 & 0.434 & 0.214 & 2.028 & 0.044 & 0.035 & 12.386 & 0.000 \\
\hline & PLS & 0.004 & 0.032 & -0.108 & 0.116 & 0.121 & 0.062 & 0.419 & 0.214 & 1.959 & 0.051 & 0.040 & 10.391 & 0.000 \\
\hline & PCR & 0.003 & 0.035 & -0.037 & -0.003 & 0.089 & 0.062 & 0.309 & 0.214 & 1.449 & 0.149 & 0.049 & 6.355 & 0.000 \\
\hline & LASSO & 0.004 & 0.017 & -0.080 & 0.180 & 0.209 & 0.062 & 0.725 & 0.216 & 3.366 & 0.001 & 0.032 & 22.390 & 0.000 \\
\hline & RIDGE & 0.003 & 0.020 & -0.185 & 0.239 & 0.131 & 0.062 & 0.454 & 0.214 & 2.123 & 0.035 & 0.040 & 11.321 & 0.000 \\
\hline & ENET & 0.003 & 0.019 & 0.004 & 0.216 & 0.163 & 0.062 & 0.565 & 0.215 & 2.633 & 0.009 & 0.042 & 13.361 & 0.000 \\
\hline & \multicolumn{14}{|l|}{ FRED-MD } \\
\hline & OLS & 0.000 & 0.006 & 0.003 & 0.102 & 0.005 & 0.062 & 0.018 & 0.213 & 0.083 & 0.934 & 0.046 & 0.384 & 0.702 \\
\hline & $3 P R F$ & 0.001 & 0.020 & 0.070 & 0.074 & 0.072 & 0.062 & 0.248 & 0.213 & 1.164 & 0.246 & 0.051 & 4.915 & 0.000 \\
\hline & PLS & 0.005 & 0.050 & -0.027 & -0.037 & 0.102 & 0.062 & 0.354 & 0.214 & 1.657 & 0.099 & 0.032 & 11.223 & 0.000 \\
\hline & PCR & 0.004 & 0.033 & -0.106 & 0.218 & 0.115 & 0.062 & 0.398 & 0.214 & 1.861 & 0.064 & 0.043 & 9.335 & 0.000 \\
\hline & LASSO & 0.004 & 0.023 & -0.084 & 0.077 & 0.156 & 0.062 & 0.540 & 0.214 & 2.517 & 0.012 & 0.041 & 13.134 & 0.000 \\
\hline & RIDGE & 0.003 & 0.026 & 0.019 & 0.013 & 0.116 & 0.062 & 0.402 & 0.214 & 1.881 & 0.061 & 0.049 & 8.250 & 0.000 \\
\hline & ENET & 0.003 & 0.023 & -0.048 & 0.051 & 0.127 & 0.062 & 0.438 & 0.214 & 2.048 & 0.042 & 0.042 & 10.477 & 0.000 \\
\hline & \multicolumn{14}{|c|}{ All Instruments } \\
\hline & OLS & 0.000 & 0.007 & -0.032 & 0.077 & -0.035 & 0.066 & -0.123 & 0.230 & -0.534 & 0.594 & 0.046 & -2.682 & 0.008 \\
\hline & $3 \mathrm{PRF}$ & 0.001 & 0.020 & 0.081 & 0.081 & 0.067 & 0.062 & 0.231 & 0.213 & 1.081 & 0.281 & 0.053 & 4.380 & 0.000 \\
\hline & PLS & 0.000 & 0.033 & 0.001 & -0.034 & 0.002 & 0.066 & 0.007 & 0.230 & 0.030 & 0.976 & 0.054 & 0.128 & 0.898 \\
\hline & PCR & 0.005 & 0.039 & -0.038 & 0.157 & 0.133 & 0.067 & 0.461 & 0.231 & 1.998 & 0.047 & 0.058 & 7.949 & 0.000 \\
\hline & LASSO & 0.003 & 0.022 & 0.000 & 0.166 & 0.122 & 0.067 & 0.424 & 0.231 & 1.837 & 0.067 & 0.052 & 8.127 & 0.000 \\
\hline & RIDGE & 0.003 & 0.026 & -0.002 & 0.035 & 0.113 & 0.067 & 0.392 & 0.231 & 1.698 & 0.091 & 0.056 & 6.944 & 0.000 \\
\hline & ENET & 0.002 & 0.023 & -0.053 & 0.146 & 0.069 & 0.066 & 0.239 & 0.230 & 1.037 & 0.301 & 0.051 & 4.638 & 0.000 \\
\hline \multicolumn{15}{|l|}{ B: } \\
\hline & & & & & & \multicolumn{2}{|c|}{ Monthly } & \multicolumn{7}{|c|}{ Annual } \\
\hline & Estimator & $\hat{\mu}$ & $\hat{\sigma}$ & $\hat{\rho}_{1}$ & $\hat{\rho}_{2}$ & $S R$ & se & $S R$ & se & $t$ & $p$ & $s e(H A C)$ & $t(H A C)$ & $p(H A C)$ \\
\hline & Goyal & & & & & & & & & & & & & \\
\hline & OLS & 0.002 & 0.019 & -0.149 & 0.086 & 0.100 & 0.062 & 0.345 & 0.214 & 1.616 & 0.107 & 0.038 & 9.176 & 0.000 \\
\hline & $3 P R F$ & 0.002 & 0.026 & -0.051 & 0.096 & 0.083 & 0.062 & 0.286 & 0.214 & 1.341 & 0.181 & 0.043 & 6.590 & 0.000 \\
\hline & PLS & 0.005 & 0.047 & -0.308 & 0.220 & 0.109 & 0.062 & 0.378 & 0.214 & 1.767 & 0.078 & 0.030 & 12.410 & 0.000 \\
\hline & PCR & 0.003 & 0.034 & 0.091 & -0.072 & 0.076 & 0.062 & 0.264 & 0.214 & 1.239 & 0.217 & 0.055 & 4.795 & 0.000 \\
\hline & LASSO & 0.002 & 0.016 & -0.132 & 0.061 & 0.141 & 0.062 & 0.488 & 0.214 & 2.278 & 0.024 & 0.036 & 13.381 & 0.000 \\
\hline & RIDGE & 0.002 & 0.018 & -0.148 & 0.082 & 0.112 & 0.062 & 0.387 & 0.214 & 1.809 & 0.072 & 0.040 & 9.716 & 0.000 \\
\hline & ENET & 0.002 & 0.017 & -0.150 & 0.192 & 0.130 & 0.062 & 0.449 & 0.214 & 2.098 & 0.037 & 0.041 & 11.050 & 0.000 \\
\hline & FRED-MD & & & & & & & & & & & & & \\
\hline & OLS & 0.000 & 0.005 & -0.045 & 0.100 & 0.009 & 0.062 & 0.032 & 0.213 & 0.150 & 0.881 & 0.044 & 0.735 & 0.463 \\
\hline & $3 P R F$ & 0.000 & 0.017 & 0.007 & 0.088 & 0.009 & 0.062 & 0.031 & 0.213 & 0.147 & 0.883 & 0.046 & 0.676 & 0.499 \\
\hline & PLS & 0.002 & 0.034 & 0.079 & -0.103 & 0.046 & 0.062 & 0.160 & 0.213 & 0.749 & 0.455 & 0.055 & 2.915 & 0.004 \\
\hline & PCR & 0.001 & 0.044 & -0.109 & 0.190 & 0.019 & 0.062 & 0.064 & 0.213 & 0.301 & 0.764 & 0.043 & 1.480 & 0.140 \\
\hline & LASSO & 0.001 & 0.019 & -0.125 & 0.012 & 0.056 & 0.062 & 0.194 & 0.213 & 0.911 & 0.363 & 0.040 & 4.893 & 0.000 \\
\hline & RIDGE & 0.001 & 0.025 & -0.038 & -0.011 & 0.028 & 0.062 & 0.095 & 0.213 & 0.448 & 0.655 & 0.046 & 2.056 & 0.041 \\
\hline & ENET & 0.001 & 0.021 & -0.104 & 0.025 & 0.032 & 0.062 & 0.112 & 0.213 & 0.524 & 0.601 & 0.040 & 2.772 & 0.006 \\
\hline & All Instrumer & & & & & & & & & & & & & \\
\hline & OLS & 0.000 & 0.005 & -0.101 & 0.101 & -0.002 & 0.066 & -0.006 & 0.230 & -0.027 & 0.979 & 0.049 & -0.126 & 0.900 \\
\hline & $3 \mathrm{PRF}$ & 0.001 & 0.018 & 0.020 & 0.162 & 0.029 & 0.062 & 0.100 & 0.213 & 0.468 & 0.640 & 0.046 & 2.151 & 0.032 \\
\hline & PLS & 0.001 & 0.023 & -0.028 & -0.049 & 0.037 & 0.066 & 0.127 & 0.230 & 0.552 & 0.581 & 0.055 & 2.303 & 0.022 \\
\hline & PCR & 0.002 & 0.047 & -0.075 & 0.171 & 0.040 & 0.066 & 0.137 & 0.230 & 0.596 & 0.552 & 0.053 & 2.586 & 0.010 \\
\hline & LASSO & 0.001 & 0.019 & -0.110 & 0.167 & 0.052 & 0.066 & 0.180 & 0.230 & 0.781 & 0.435 & 0.048 & 3.713 & 0.000 \\
\hline & RIDGE & 0.001 & 0.028 & -0.097 & 0.003 & 0.035 & 0.066 & 0.120 & 0.230 & 0.522 & 0.602 & 0.049 & 2.463 & 0.015 \\
\hline & ENET & 0.001 & 0.018 & -0.105 & 0.051 & 0.047 & 0.066 & 0.162 & 0.230 & 0.702 & 0.483 & 0.047 & 3.417 & 0.001 \\
\hline
\end{tabular}

(Continued) 
Table C.2: Results for the 5 Industry Portfolios (Continued)

\begin{tabular}{|c|c|c|c|c|c|c|c|c|c|c|c|c|c|}
\hline \multirow[b]{2}{*}{ Estimator } & \multirow[b]{2}{*}{$\hat{\mu}$} & \multirow[b]{2}{*}{$\hat{\sigma}$} & \multirow[b]{2}{*}{$\hat{\rho}_{1}$} & \multirow[b]{2}{*}{$\hat{\rho}_{2}$} & \multicolumn{2}{|c|}{ Monthly } & \multicolumn{7}{|c|}{ Annual } \\
\hline & & & & & $S R$ & se & $S R$ & se & $t$ & $p$ & $s e(H A C)$ & $t(H A C)$ & $p(H A C)$ \\
\hline \multicolumn{14}{|l|}{ Goyal } \\
\hline OLS & 0.004 & 0.030 & -0.121 & 0.015 & 0.135 & 0.062 & 0.467 & 0.214 & 2.181 & 0.030 & 0.037 & 12.513 & 0.000 \\
\hline $3 \mathrm{PRF}$ & 0.005 & 0.042 & -0.107 & 0.091 & 0.125 & 0.062 & 0.435 & 0.214 & 2.030 & 0.043 & 0.035 & 12.492 & 0.000 \\
\hline PLS & 0.007 & 0.062 & -0.107 & 0.114 & 0.119 & 0.062 & 0.414 & 0.214 & 1.934 & 0.054 & 0.040 & 10.274 & 0.000 \\
\hline PCR & 0.006 & 0.068 & -0.038 & -0.007 & 0.085 & 0.062 & 0.295 & 0.214 & 1.383 & 0.168 & 0.049 & 6.076 & 0.000 \\
\hline LASSO & 0.007 & 0.033 & -0.082 & 0.184 & 0.209 & 0.062 & 0.723 & 0.216 & 3.355 & 0.001 & 0.031 & 23.150 & 0.000 \\
\hline RIDGE & 0.005 & 0.039 & -0.192 & 0.243 & 0.131 & 0.062 & 0.455 & 0.214 & 2.125 & 0.034 & 0.040 & 11.503 & 0.000 \\
\hline ENET & 0.006 & 0.037 & -0.001 & 0.222 & 0.168 & 0.062 & 0.581 & 0.215 & 2.704 & 0.007 & 0.037 & 15.738 & 0.000 \\
\hline \multicolumn{14}{|l|}{ FRED-MD } \\
\hline OLS & -0.001 & 0.014 & 0.019 & 0.115 & -0.046 & 0.062 & -0.160 & 0.213 & -0.748 & 0.455 & 0.044 & -3.608 & 0.000 \\
\hline $3 \mathrm{PRF}$ & 0.003 & 0.038 & 0.071 & 0.080 & 0.073 & 0.062 & 0.252 & 0.213 & 1.180 & 0.239 & 0.050 & 5.011 & 0.000 \\
\hline PLS & 0.010 & 0.097 & -0.026 & -0.038 & 0.101 & 0.062 & 0.351 & 0.214 & 1.640 & 0.102 & 0.032 & 11.038 & 0.000 \\
\hline PCR & 0.007 & 0.063 & -0.104 & 0.221 & 0.112 & 0.062 & 0.389 & 0.214 & 1.821 & 0.070 & 0.043 & 9.116 & 0.000 \\
\hline LASSO & 0.007 & 0.044 & -0.081 & 0.074 & 0.152 & 0.062 & 0.528 & 0.214 & 2.461 & 0.014 & 0.041 & 12.803 & 0.000 \\
\hline RIDGE & 0.006 & 0.051 & 0.022 & 0.013 & 0.116 & 0.062 & 0.401 & 0.214 & 1.873 & 0.062 & 0.049 & 8.174 & 0.000 \\
\hline ENET & 0.005 & 0.044 & -0.036 & 0.044 & 0.125 & 0.062 & 0.434 & 0.214 & 2.026 & 0.044 & 0.043 & 10.166 & 0.000 \\
\hline \multicolumn{14}{|c|}{ All Instruments } \\
\hline OLS & -0.001 & 0.018 & 0.022 & 0.136 & -0.078 & 0.066 & -0.271 & 0.230 & -1.179 & 0.240 & 0.042 & -6.499 & 0.000 \\
\hline $3 \mathrm{PRF}$ & 0.003 & 0.039 & 0.084 & 0.082 & 0.068 & 0.062 & 0.235 & 0.213 & 1.102 & 0.271 & 0.053 & 4.459 & 0.000 \\
\hline PLS & 0.000 & 0.065 & 0.002 & -0.038 & -0.001 & 0.066 & -0.003 & 0.230 & -0.011 & 0.991 & 0.053 & -0.048 & 0.962 \\
\hline PCR & 0.010 & 0.075 & -0.035 & 0.154 & 0.129 & 0.067 & 0.447 & 0.231 & 1.936 & 0.054 & 0.058 & 7.669 & 0.000 \\
\hline LASSO & 0.005 & 0.043 & 0.003 & 0.172 & 0.118 & 0.067 & 0.408 & 0.231 & 1.768 & 0.078 & 0.052 & 7.811 & 0.000 \\
\hline RIDGE & 0.006 & 0.051 & 0.003 & 0.036 & 0.112 & 0.067 & 0.388 & 0.231 & 1.680 & 0.094 & 0.056 & 6.868 & 0.000 \\
\hline ENET & 0.003 & 0.044 & -0.045 & 0.147 & 0.069 & 0.066 & 0.239 & 0.230 & 1.038 & 0.300 & 0.052 & 4.586 & 0.000 \\
\hline
\end{tabular}

Table C.2 2 reports some summary statistics of the OOS (Jan-1996 to Dec-2017) for the 5 Industry portfolios by estimator and three different set of conditioning information (Goyal, FRED-MD and "All Instruments"). Each panel shows the test of pairs of Sharpe ratios for three different framework to build efficient portfolios. Panel A reports conditionally mean-variance (CMV) efficient portfolios. Panel B reports unconditionally mean-variance efficient portfolios (UMV). Panel C presents the mean-variance tracking error (MVATE) portfolios. We split the results depending on conditioning information set used. Goyal variables comprises: $b / m, d f r, d f y$, infl, ltr, lty, ntis, svar, tms and tbl. The FRED-MD is a large dataset containing 128 macroeconomic and financial variables. Finally, "All Instruments" is the combination of Goyal and FREDMD datasets with the Economic Policy Uncertainty (EPU) index and the Financial Stress Indicator (FSI). The table reports the monthly returns $(\hat{\mu})$, monthly returns $(\hat{\sigma})$, first and second autocorrelation $\left(\hat{\rho}_{1}\right.$ and $\left.\hat{\rho}_{2}\right)$ of the monthly returns. The Sharpe ratios $(S R)$ are presented monthly and annualized, as well as the standard error $(\mathrm{se})$. For the sake of completeness, it is also reported the t-statistic $(t)$, the standard p-value $(p)$, as well as the HAC standard error $(s e(H A C))$, the t-statistic obtained using HAC $(t(H A C))$ and its p-value $(p(H A C))$. 
Table C.3: Results for the 6 Portfolios Formed on Size and Book-to-Market

\begin{tabular}{|c|c|c|c|c|c|c|c|c|c|c|c|c|c|c|}
\hline \multirow[t]{3}{*}{ A: } & \multicolumn{14}{|l|}{ CMV } \\
\hline & \multirow[b]{2}{*}{ Estimator } & \multirow[b]{2}{*}{$\hat{\mu}$} & \multirow[b]{2}{*}{$\hat{\sigma}$} & \multirow[b]{2}{*}{$\hat{\rho}_{1}$} & \multirow[b]{2}{*}{$\hat{\rho}_{2}$} & \multicolumn{2}{|c|}{ Monthly } & \multicolumn{7}{|c|}{ Annual } \\
\hline & & & & & & $S R$ & se & $S R$ & se & $t$ & $p$-val & $s e(H A C)$ & $t(H A C)$ & $p(H A C)$ \\
\hline & \multicolumn{14}{|l|}{ Goyal } \\
\hline & OLS & 0.002 & 0.012 & 0.057 & -0.024 & 0.207 & 0.062 & 0.719 & 0.215 & 3.335 & 0.001 & 0.049 & 14.647 & 0.000 \\
\hline & $3 \mathrm{PRF}$ & 0.002 & 0.010 & 0.160 & 0.031 & 0.222 & 0.062 & 0.769 & 0.216 & 3.563 & 0.000 & 0.052 & 14.904 & 0.000 \\
\hline & PLS & 0.004 & 0.015 & 0.158 & -0.016 & 0.247 & 0.062 & 0.856 & 0.216 & 3.954 & 0.000 & 0.059 & 14.417 & 0.000 \\
\hline & PCR & 0.005 & 0.016 & 0.132 & 0.024 & 0.298 & 0.063 & 1.032 & 0.218 & 4.738 & 0.000 & 0.048 & 21.518 & 0.000 \\
\hline & LASSO & 0.002 & 0.013 & 0.030 & -0.002 & 0.168 & 0.062 & 0.580 & 0.215 & 2.704 & 0.007 & 0.046 & 12.726 & 0.000 \\
\hline & RIDGE & 0.003 & 0.015 & 0.131 & -0.024 & 0.173 & 0.062 & 0.600 & 0.215 & 2.793 & 0.006 & 0.059 & 10.197 & 0.000 \\
\hline & ENET & 0.003 & 0.012 & 0.041 & -0.097 & 0.231 & 0.062 & 0.799 & 0.216 & 3.698 & 0.000 & 0.045 & 17.752 & 0.000 \\
\hline & \multicolumn{14}{|l|}{ FRED-MD } \\
\hline & OLS & 0.001 & 0.005 & 0.050 & 0.024 & 0.154 & 0.062 & 0.534 & 0.214 & 2.491 & 0.013 & 0.049 & 10.802 & 0.000 \\
\hline & $3 \mathrm{PRF}$ & 0.003 & 0.014 & -0.018 & -0.066 & 0.186 & 0.062 & 0.646 & 0.215 & 3.003 & 0.003 & 0.048 & 13.522 & 0.000 \\
\hline & PLS & 0.003 & 0.016 & 0.142 & 0.046 & 0.210 & 0.062 & 0.726 & 0.216 & 3.369 & 0.001 & 0.052 & 13.883 & 0.000 \\
\hline & PCR & 0.005 & 0.017 & 0.127 & 0.098 & 0.272 & 0.063 & 0.944 & 0.217 & 4.346 & 0.000 & 0.044 & 21.651 & 0.000 \\
\hline & LASSO & 0.004 & 0.016 & -0.039 & -0.057 & 0.226 & 0.062 & 0.783 & 0.216 & 3.627 & 0.000 & 0.033 & 23.946 & 0.000 \\
\hline & RIDGE & 0.004 & 0.016 & 0.037 & -0.029 & 0.242 & 0.062 & 0.840 & 0.216 & 3.881 & 0.000 & 0.047 & 17.773 & 0.000 \\
\hline & ENET & 0.004 & 0.015 & 0.054 & -0.027 & 0.261 & 0.063 & 0.905 & 0.217 & 4.172 & 0.000 & 0.040 & 22.576 & 0.000 \\
\hline & \multicolumn{14}{|c|}{ All Instruments } \\
\hline & OLS & 0.001 & 0.004 & 0.097 & -0.017 & 0.160 & 0.067 & 0.554 & 0.231 & 2.395 & 0.017 & 0.065 & 8.465 & 0.000 \\
\hline & $3 \mathrm{PRF}$ & 0.003 & 0.013 & 0.001 & -0.086 & 0.193 & 0.062 & 0.670 & 0.215 & 3.113 & 0.002 & 0.047 & 14.340 & 0.000 \\
\hline & PLS & 0.004 & 0.014 & 0.122 & 0.087 & 0.264 & 0.068 & 0.916 & 0.234 & 3.915 & 0.000 & 0.063 & 14.545 & 0.000 \\
\hline & PCR & 0.005 & 0.019 & 0.136 & 0.104 & 0.238 & 0.067 & 0.825 & 0.233 & 3.539 & 0.000 & 0.054 & 15.290 & 0.000 \\
\hline & LASSO & 0.004 & 0.014 & -0.019 & -0.044 & 0.271 & 0.063 & 0.940 & 0.219 & 4.296 & 0.000 & 0.038 & 24.768 & 0.000 \\
\hline & RIDGE & 0.004 & 0.015 & 0.034 & -0.016 & 0.248 & 0.067 & 0.858 & 0.233 & 3.674 & 0.000 & 0.054 & 15.940 & 0.000 \\
\hline & ENET & 0.003 & 0.013 & -0.011 & -0.035 & 0.255 & 0.063 & 0.883 & 0.218 & 4.052 & 0.000 & 0.040 & 21.857 & 0.000 \\
\hline \multicolumn{15}{|c|}{ UMV } \\
\hline & & & & & & \multicolumn{2}{|c|}{ Monthly } & \multicolumn{7}{|c|}{ Annual } \\
\hline & Estimator & $\hat{\mu}$ & $\hat{\sigma}$ & $\hat{\rho}_{1}$ & $\hat{\rho}_{2}$ & $S R$ & se & $S R$ & se & $t$ & $p$ & $s e(H A C)$ & $t(H A C)$ & $p(H A C)$ \\
\hline & Goyal & & & & & & & & & & & & & \\
\hline & OLS & 0.002 & 0.010 & 0.055 & -0.110 & 0.218 & 0.062 & 0.757 & 0.216 & 3.508 & 0.001 & 0.046 & 16.626 & 0.000 \\
\hline & $3 \mathrm{PRF}$ & 0.003 & 0.011 & 0.144 & 0.000 & 0.258 & 0.063 & 0.893 & 0.217 & 4.122 & 0.000 & 0.046 & 19.347 & 0.000 \\
\hline & PLS & 0.004 & 0.017 & 0.139 & 0.111 & 0.246 & 0.062 & 0.853 & 0.216 & 3.941 & 0.000 & 0.054 & 15.805 & 0.000 \\
\hline & PCR & 0.005 & 0.016 & 0.167 & 0.083 & 0.299 & 0.063 & 1.035 & 0.218 & 4.749 & 0.000 & 0.052 & 19.905 & 0.000 \\
\hline & LASSO & 0.002 & 0.009 & 0.041 & -0.071 & 0.187 & 0.062 & 0.648 & 0.215 & 3.011 & 0.003 & 0.049 & 13.293 & 0.000 \\
\hline & RIDGE & 0.002 & 0.011 & 0.077 & -0.040 & 0.224 & 0.062 & 0.774 & 0.216 & 3.587 & 0.000 & 0.048 & 16.120 & 0.000 \\
\hline & ENET & 0.002 & 0.009 & 0.029 & -0.074 & 0.260 & 0.063 & 0.901 & 0.217 & 4.157 & 0.000 & 0.044 & 20.464 & 0.000 \\
\hline & FRED-MD & & & & & & & & & & & & & \\
\hline & OLS & 0.001 & 0.004 & 0.116 & 0.043 & 0.158 & 0.062 & 0.546 & 0.215 & 2.547 & 0.011 & 0.057 & 9.636 & 0.000 \\
\hline & $3 \mathrm{PRF}$ & 0.002 & 0.012 & 0.135 & -0.021 & 0.197 & 0.062 & 0.681 & 0.215 & 3.163 & 0.002 & 0.060 & 11.418 & 0.000 \\
\hline & PLS & 0.003 & 0.016 & 0.150 & 0.076 & 0.198 & 0.062 & 0.687 & 0.215 & 3.189 & 0.002 & 0.058 & 11.751 & 0.000 \\
\hline & PCR & 0.004 & 0.017 & 0.118 & 0.055 & 0.255 & 0.063 & 0.882 & 0.217 & 4.070 & 0.000 & 0.059 & 14.887 & 0.000 \\
\hline & LASSO & 0.002 & 0.010 & 0.098 & 0.017 & 0.201 & 0.062 & 0.697 & 0.215 & 3.239 & 0.001 & 0.046 & 15.132 & 0.000 \\
\hline & RIDGE & 0.003 & 0.014 & 0.142 & -0.021 & 0.230 & 0.062 & 0.797 & 0.216 & 3.688 & 0.000 & 0.054 & 14.801 & 0.000 \\
\hline & ENET & 0.003 & 0.012 & 0.138 & 0.047 & 0.261 & 0.063 & 0.905 & 0.217 & 4.173 & 0.000 & 0.044 & 20.408 & 0.000 \\
\hline & All Instrume & & & & & & & & & & & & & \\
\hline & OLS & 0.001 & 0.004 & 0.125 & -0.007 & 0.168 & 0.067 & 0.583 & 0.232 & 2.519 & 0.012 & 0.070 & 8.374 & 0.000 \\
\hline & $3 \mathrm{PRF}$ & 0.003 & 0.012 & 0.167 & -0.027 & 0.213 & 0.062 & 0.738 & 0.216 & 3.421 & 0.001 & 0.059 & 12.574 & 0.000 \\
\hline & PLS & 0.004 & 0.014 & 0.104 & 0.032 & 0.263 & 0.068 & 0.911 & 0.234 & 3.897 & 0.000 & 0.060 & 15.097 & 0.000 \\
\hline & PCR & 0.004 & 0.017 & 0.133 & 0.053 & 0.237 & 0.067 & 0.820 & 0.233 & 3.517 & 0.001 & 0.069 & 11.858 & 0.000 \\
\hline & LASSO & 0.002 & 0.009 & 0.069 & -0.026 & 0.228 & 0.063 & 0.790 & 0.218 & 3.628 & 0.000 & 0.043 & 18.550 & 0.000 \\
\hline & RIDGE & 0.003 & 0.014 & 0.147 & -0.021 & 0.229 & 0.067 & 0.794 & 0.233 & 3.407 & 0.001 & 0.060 & 13.331 & 0.000 \\
\hline & ENET & 0.002 & 0.009 & 0.038 & -0.045 & 0.234 & 0.063 & 0.812 & 0.217 & 3.736 & 0.000 & 0.043 & 19.023 & 0.000 \\
\hline
\end{tabular}


Table C.3: Results for the 6 Portfolios Formed on Size and Book-to-Market (Continued)

C: MVATE

\begin{tabular}{|c|c|c|c|c|c|c|c|c|c|c|c|c|c|}
\hline \multirow[b]{2}{*}{ Estimator } & \multirow[b]{2}{*}{$\hat{\mu}$} & \multirow[b]{2}{*}{$\hat{\sigma}$} & \multirow[b]{2}{*}{$\hat{\rho}_{1}$} & \multirow[b]{2}{*}{$\hat{\rho}_{2}$} & \multicolumn{2}{|c|}{ Monthly } & \multicolumn{7}{|c|}{ Annual } \\
\hline & & & & & $S R$ & se & $S R$ & se & $t$ & $p$ & $s e(H A C)$ & $t(H A C)$ & $p(H A C)$ \\
\hline \multicolumn{14}{|l|}{ Goyal } \\
\hline OLS & 0.004 & 0.022 & 0.059 & -0.025 & 0.203 & 0.062 & 0.703 & 0.215 & 3.266 & 0.001 & 0.050 & 14.175 & 0.000 \\
\hline $3 \mathrm{PRF}$ & 0.004 & 0.020 & 0.161 & 0.034 & 0.223 & 0.062 & 0.773 & 0.216 & 3.583 & 0.000 & 0.052 & 14.856 & 0.000 \\
\hline PLS & 0.007 & 0.029 & 0.169 & -0.011 & 0.247 & 0.062 & 0.857 & 0.216 & 3.958 & 0.000 & 0.061 & 14.037 & 0.000 \\
\hline PCR & 0.009 & 0.031 & 0.144 & 0.034 & 0.300 & 0.063 & 1.039 & 0.218 & 4.768 & 0.000 & 0.048 & 21.704 & 0.000 \\
\hline LASSO & 0.004 & 0.025 & 0.027 & -0.010 & 0.166 & 0.062 & 0.575 & 0.215 & 2.678 & 0.008 & 0.046 & 12.624 & 0.000 \\
\hline RIDGE & 0.005 & 0.029 & 0.132 & -0.022 & 0.174 & 0.062 & 0.604 & 0.215 & 2.811 & 0.005 & 0.060 & 10.138 & 0.000 \\
\hline ENET & 0.006 & 0.024 & 0.033 & -0.098 & 0.236 & 0.062 & 0.816 & 0.216 & 3.774 & 0.000 & 0.044 & 18.609 & 0.000 \\
\hline \multicolumn{14}{|l|}{ FRED-MD } \\
\hline OLS & 0.001 & 0.014 & -0.175 & -0.006 & 0.037 & 0.062 & 0.128 & 0.213 & 0.599 & 0.550 & 0.035 & 3.648 & 0.000 \\
\hline $3 \mathrm{PRF}$ & 0.005 & 0.027 & -0.019 & -0.064 & 0.188 & 0.062 & 0.652 & 0.215 & 3.031 & 0.003 & 0.048 & 13.619 & 0.000 \\
\hline PLS & 0.006 & 0.032 & 0.138 & 0.037 & 0.201 & 0.062 & 0.695 & 0.215 & 3.226 & 0.001 & 0.051 & 13.732 & 0.000 \\
\hline PCR & 0.009 & 0.033 & 0.131 & 0.105 & 0.275 & 0.063 & 0.951 & 0.217 & 4.378 & 0.000 & 0.043 & 22.089 & 0.000 \\
\hline LASSO & 0.007 & 0.030 & -0.039 & -0.053 & 0.226 & 0.062 & 0.783 & 0.216 & 3.625 & 0.000 & 0.032 & 24.708 & 0.000 \\
\hline RIDGE & 0.007 & 0.030 & 0.036 & -0.023 & 0.246 & 0.062 & 0.850 & 0.216 & 3.930 & 0.000 & 0.047 & 18.052 & 0.000 \\
\hline ENET & 0.008 & 0.029 & 0.050 & -0.018 & 0.261 & 0.063 & 0.905 & 0.217 & 4.173 & 0.000 & 0.039 & 23.380 & 0.000 \\
\hline \multicolumn{14}{|c|}{ All Instruments } \\
\hline OLS & 0.000 & 0.018 & -0.130 & -0.057 & -0.002 & 0.066 & -0.008 & 0.230 & -0.034 & 0.973 & 0.042 & -0.184 & 0.854 \\
\hline $3 \mathrm{PRF}$ & 0.005 & 0.025 & -0.001 & -0.087 & 0.191 & 0.062 & 0.661 & 0.215 & 3.072 & 0.002 & 0.048 & 13.744 & 0.000 \\
\hline PLS & 0.007 & 0.028 & 0.132 & 0.095 & 0.265 & 0.068 & 0.917 & 0.234 & 3.921 & 0.000 & 0.064 & 14.399 & 0.000 \\
\hline PCR & 0.009 & 0.037 & 0.143 & 0.106 & 0.240 & 0.067 & 0.831 & 0.233 & 3.565 & 0.000 & 0.054 & 15.490 & 0.000 \\
\hline LASSO & 0.007 & 0.026 & -0.019 & -0.038 & 0.274 & 0.063 & 0.950 & 0.219 & 4.339 & 0.000 & 0.037 & 25.655 & 0.000 \\
\hline RIDGE & 0.007 & 0.029 & 0.044 & -0.016 & 0.247 & 0.067 & 0.856 & 0.233 & 3.669 & 0.000 & 0.054 & 15.731 & 0.000 \\
\hline ENET & 0.007 & 0.026 & -0.026 & -0.033 & 0.266 & 0.063 & 0.921 & 0.219 & 4.206 & 0.000 & 0.039 & 23.441 & 0.000 \\
\hline
\end{tabular}

Table C.3 reports some summary statistics of the OOS (Jan-1996 to Dec-2017) for the 6 portfolios formed on size and book-to-market by estimator and three different set of conditioning information (Goyal, FRED-MD and "All Instruments"). Each panel shows the test of pairs of Sharpe ratios for three different framework to build efficient portfolios. Panel A reports conditionally mean-variance (CMV) efficient portfolios. Panel B reports unconditionally mean-variance efficient portfolios (UMV). Panel C presents the mean-variance tracking error (MVATE) portfolios. We split the results depending on conditioning information set used. Goyal variables comprises: $b / m$, $d f r, d f y$, infl, ltr, lty, ntis, svar, tms and $t b l$. The FRED-MD is a large dataset containing 128 macroeconomic and financial variables. Finally, "All Instruments" is the combination of Goyal and FRED-MD datasets with the Economic Policy Uncertainty (EPU) index and the Financial Stress Indicator (FSI). The table reports the monthly returns $(\hat{\mu})$, monthly returns $(\hat{\sigma})$, first and second autocorrelation ( $\hat{\rho}_{1}$ and $\hat{\rho}_{2}$ ) of the monthly returns. The Sharpe ratios $(S R)$ are presented monthly and annualized, as well as the standard error $(\mathrm{se})$. For the sake of completeness, it is also reported the t-statistic $(t)$, the standard p-value $(p)$, as well as the HAC standard error $(s e(H A C))$, the t-statistic obtained using HAC $(t(H A C))$ and its p-value $(p(H A C))$. 
Table C.4: Results for the 25 Portfolios Formed on Size and Book-to-Market

\begin{tabular}{|c|c|c|c|c|c|c|c|c|c|c|c|c|c|c|}
\hline \multirow[t]{3}{*}{ A: } & \multicolumn{14}{|l|}{ CMV } \\
\hline & \multirow[b]{2}{*}{ Estimator } & \multirow[b]{2}{*}{$\hat{\mu}$} & \multirow[b]{2}{*}{$\hat{\sigma}$} & \multirow[b]{2}{*}{$\hat{\rho}_{1}$} & \multirow[b]{2}{*}{$\hat{\rho}_{2}$} & \multicolumn{2}{|c|}{ Monthly } & \multicolumn{7}{|c|}{ Annual } \\
\hline & & & & & & $S R$ & se & $S R$ & se & $t$ & $p$-val & $s e(H A C)$ & $t(H A C)$ & $p(H A C)$ \\
\hline & \multicolumn{14}{|l|}{ Goyal } \\
\hline & OLS & 0.002 & 0.007 & -0.108 & 0.124 & 0.307 & 0.063 & 1.062 & 0.218 & 4.868 & 0.000 & 0.038 & 27.935 & 0.000 \\
\hline & $3 \mathrm{PRF}$ & 0.002 & 0.007 & 0.005 & 0.044 & 0.327 & 0.063 & 1.132 & 0.219 & 5.171 & 0.000 & 0.044 & 25.789 & 0.000 \\
\hline & PLS & 0.003 & 0.010 & 0.017 & 0.046 & 0.307 & 0.063 & 1.063 & 0.218 & 4.870 & 0.000 & 0.046 & 23.139 & 0.000 \\
\hline & PCR & 0.004 & 0.011 & 0.092 & -0.042 & 0.383 & 0.064 & 1.325 & 0.221 & 6.000 & 0.000 & 0.054 & 24.516 & 0.000 \\
\hline & LASSO & 0.002 & 0.006 & 0.016 & 0.167 & 0.276 & 0.063 & 0.957 & 0.217 & 4.407 & 0.000 & 0.047 & 20.181 & 0.000 \\
\hline & RIDGE & 0.003 & 0.008 & -0.130 & 0.147 & 0.313 & 0.063 & 1.083 & 0.218 & 4.961 & 0.000 & 0.039 & 27.911 & 0.000 \\
\hline & ENET & 0.002 & 0.006 & -0.123 & 0.155 & 0.315 & 0.063 & 1.092 & 0.218 & 4.998 & 0.000 & 0.036 & 30.736 & 0.000 \\
\hline & \multicolumn{14}{|l|}{ FRED-MD } \\
\hline & OLS & 0.000 & 0.002 & -0.061 & -0.031 & 0.161 & 0.062 & 0.559 & 0.215 & 2.605 & 0.010 & 0.045 & 12.313 & 0.000 \\
\hline & $3 \mathrm{PRF}$ & 0.002 & 0.007 & 0.096 & -0.006 & 0.282 & 0.063 & 0.978 & 0.217 & 4.497 & 0.000 & 0.052 & 18.951 & 0.000 \\
\hline & PLS & 0.003 & 0.009 & 0.026 & 0.057 & 0.296 & 0.063 & 1.024 & 0.218 & 4.701 & 0.000 & 0.043 & 23.716 & 0.000 \\
\hline & PCR & 0.004 & 0.011 & 0.079 & 0.048 & 0.371 & 0.064 & 1.286 & 0.220 & 5.834 & 0.000 & 0.052 & 24.585 & 0.000 \\
\hline & LASSO & 0.002 & 0.008 & -0.071 & -0.076 & 0.215 & 0.062 & 0.743 & 0.216 & 3.448 & 0.001 & 0.044 & 17.027 & 0.000 \\
\hline & RIDGE & 0.003 & 0.011 & 0.053 & -0.014 & 0.274 & 0.063 & 0.949 & 0.217 & 4.370 & 0.000 & 0.049 & 19.392 & 0.000 \\
\hline & ENET & 0.002 & 0.008 & -0.025 & -0.053 & 0.280 & 0.063 & 0.970 & 0.217 & 4.465 & 0.000 & 0.048 & 20.323 & 0.000 \\
\hline & \multicolumn{14}{|c|}{ All Instruments } \\
\hline & OLS & 0.000 & 0.002 & -0.005 & 0.009 & 0.201 & 0.067 & 0.696 & 0.232 & 2.996 & 0.003 & 0.053 & 13.137 & 0.000 \\
\hline & $3 \mathrm{PRF}$ & 0.002 & 0.007 & 0.076 & -0.005 & 0.290 & 0.063 & 1.006 & 0.218 & 4.621 & 0.000 & 0.050 & 19.946 & 0.000 \\
\hline & PLS & 0.003 & 0.009 & 0.080 & 0.050 & 0.331 & 0.068 & 1.145 & 0.236 & 4.849 & 0.000 & 0.053 & 21.547 & 0.000 \\
\hline & PCR & 0.004 & 0.013 & 0.007 & 0.029 & 0.307 & 0.068 & 1.063 & 0.235 & 4.518 & 0.000 & 0.055 & 19.254 & 0.000 \\
\hline & LASSO & 0.002 & 0.008 & -0.098 & 0.018 & 0.231 & 0.063 & 0.799 & 0.220 & 3.637 & 0.000 & 0.040 & 20.170 & 0.000 \\
\hline & RIDGE & 0.003 & 0.010 & 0.006 & 0.017 & 0.305 & 0.068 & 1.057 & 0.235 & 4.493 & 0.000 & 0.054 & 19.399 & 0.000 \\
\hline & ENET & 0.002 & 0.008 & -0.118 & -0.018 & 0.302 & 0.064 & 1.045 & 0.222 & 4.701 & 0.000 & 0.040 & 25.901 & 0.000 \\
\hline \multicolumn{15}{|c|}{ UMV } \\
\hline & & & & & & \multicolumn{2}{|c|}{ Monthly } & \multicolumn{7}{|c|}{ Annual } \\
\hline & Estimator & $\hat{\mu}$ & $\hat{\sigma}$ & $\hat{\rho}_{1}$ & $\hat{\rho}_{2}$ & $S R$ & se & $S R$ & se & $t$ & $p$ & $s e(H A C)$ & $t(H A C)$ & $p(H A C)$ \\
\hline & Goyal & & & & & & & & & & & & & \\
\hline & OLS & 0.002 & 0.006 & -0.078 & 0.107 & 0.293 & 0.063 & 1.017 & 0.218 & 4.668 & 0.000 & 0.037 & 27.165 & 0.000 \\
\hline & $3 \mathrm{PRF}$ & 0.002 & 0.007 & 0.033 & -0.005 & 0.330 & 0.063 & 1.143 & 0.219 & 5.219 & 0.000 & 0.042 & 27.225 & 0.000 \\
\hline & PLS & 0.003 & 0.011 & 0.099 & 0.074 & 0.322 & 0.063 & 1.114 & 0.219 & 5.094 & 0.000 & 0.041 & 27.417 & 0.000 \\
\hline & PCR & 0.004 & 0.011 & 0.121 & 0.024 & 0.386 & 0.064 & 1.337 & 0.221 & 6.049 & 0.000 & 0.050 & 26.591 & 0.000 \\
\hline & LASSO & 0.001 & 0.005 & 0.016 & 0.102 & 0.279 & 0.063 & 0.968 & 0.217 & 4.453 & 0.000 & 0.046 & 20.973 & 0.000 \\
\hline & RIDGE & 0.002 & 0.007 & -0.075 & 0.042 & 0.311 & 0.063 & 1.076 & 0.218 & 4.928 & 0.000 & 0.040 & 26.583 & 0.000 \\
\hline & ENET & 0.002 & 0.005 & -0.066 & 0.088 & 0.316 & 0.063 & 1.095 & 0.218 & 5.014 & 0.000 & 0.037 & 29.458 & 0.000 \\
\hline & FRED-MD & & & & & & & & & & & & & \\
\hline & OLS & 0.000 & 0.002 & -0.058 & -0.023 & 0.166 & 0.062 & 0.576 & 0.215 & 2.683 & 0.008 & 0.045 & 12.696 & 0.000 \\
\hline & $3 \mathrm{PRF}$ & 0.002 & 0.007 & 0.113 & -0.001 & 0.262 & 0.063 & 0.907 & 0.217 & 4.182 & 0.000 & 0.059 & 15.475 & 0.000 \\
\hline & PLS & 0.003 & 0.009 & 0.089 & 0.057 & 0.299 & 0.063 & 1.036 & 0.218 & 4.756 & 0.000 & 0.043 & 24.224 & 0.000 \\
\hline & PCR & 0.004 & 0.011 & 0.072 & 0.042 & 0.361 & 0.064 & 1.252 & 0.220 & 5.687 & 0.000 & 0.051 & 24.710 & 0.000 \\
\hline & LASSO & 0.002 & 0.007 & -0.062 & -0.106 & 0.209 & 0.062 & 0.724 & 0.216 & 3.357 & 0.001 & 0.048 & 15.114 & 0.000 \\
\hline & RIDGE & 0.003 & 0.010 & 0.135 & -0.047 & 0.277 & 0.063 & 0.960 & 0.217 & 4.417 & 0.000 & 0.051 & 18.862 & 0.000 \\
\hline & ENET & 0.002 & 0.007 & -0.017 & -0.123 & 0.267 & 0.063 & 0.924 & 0.217 & 4.259 & 0.000 & 0.052 & 17.747 & 0.000 \\
\hline & All Instrume & & & & & & & & & & & & & \\
\hline & OLS & 0.000 & 0.002 & 0.001 & 0.011 & 0.204 & 0.067 & 0.706 & 0.232 & 3.039 & 0.003 & 0.054 & 13.132 & 0.000 \\
\hline & $3 \mathrm{PRF}$ & 0.002 & 0.007 & 0.105 & -0.007 & 0.278 & 0.063 & 0.964 & 0.217 & 4.436 & 0.000 & 0.054 & 17.895 & 0.000 \\
\hline & PLS & 0.003 & 0.009 & 0.115 & -0.014 & 0.303 & 0.068 & 1.051 & 0.235 & 4.469 & 0.000 & 0.053 & 19.951 & 0.000 \\
\hline & PCR & 0.004 & 0.012 & 0.046 & 0.019 & 0.293 & 0.068 & 1.015 & 0.235 & 4.322 & 0.000 & 0.062 & 16.363 & 0.000 \\
\hline & LASSO & 0.001 & 0.006 & -0.070 & 0.014 & 0.230 & 0.063 & 0.797 & 0.220 & 3.628 & 0.000 & 0.043 & 18.443 & 0.000 \\
\hline & RIDGE & 0.003 & 0.010 & 0.101 & -0.020 & 0.306 & 0.068 & 1.061 & 0.235 & 4.511 & 0.000 & 0.057 & 18.572 & 0.000 \\
\hline & ENET & 0.002 & 0.006 & -0.066 & -0.057 & 0.280 & 0.064 & 0.970 & 0.222 & 4.376 & 0.000 & 0.047 & 20.478 & 0.000 \\
\hline
\end{tabular}


Table C.4: Results for the 25 Portfolios Formed on Size and Book-to-Market (Continued)

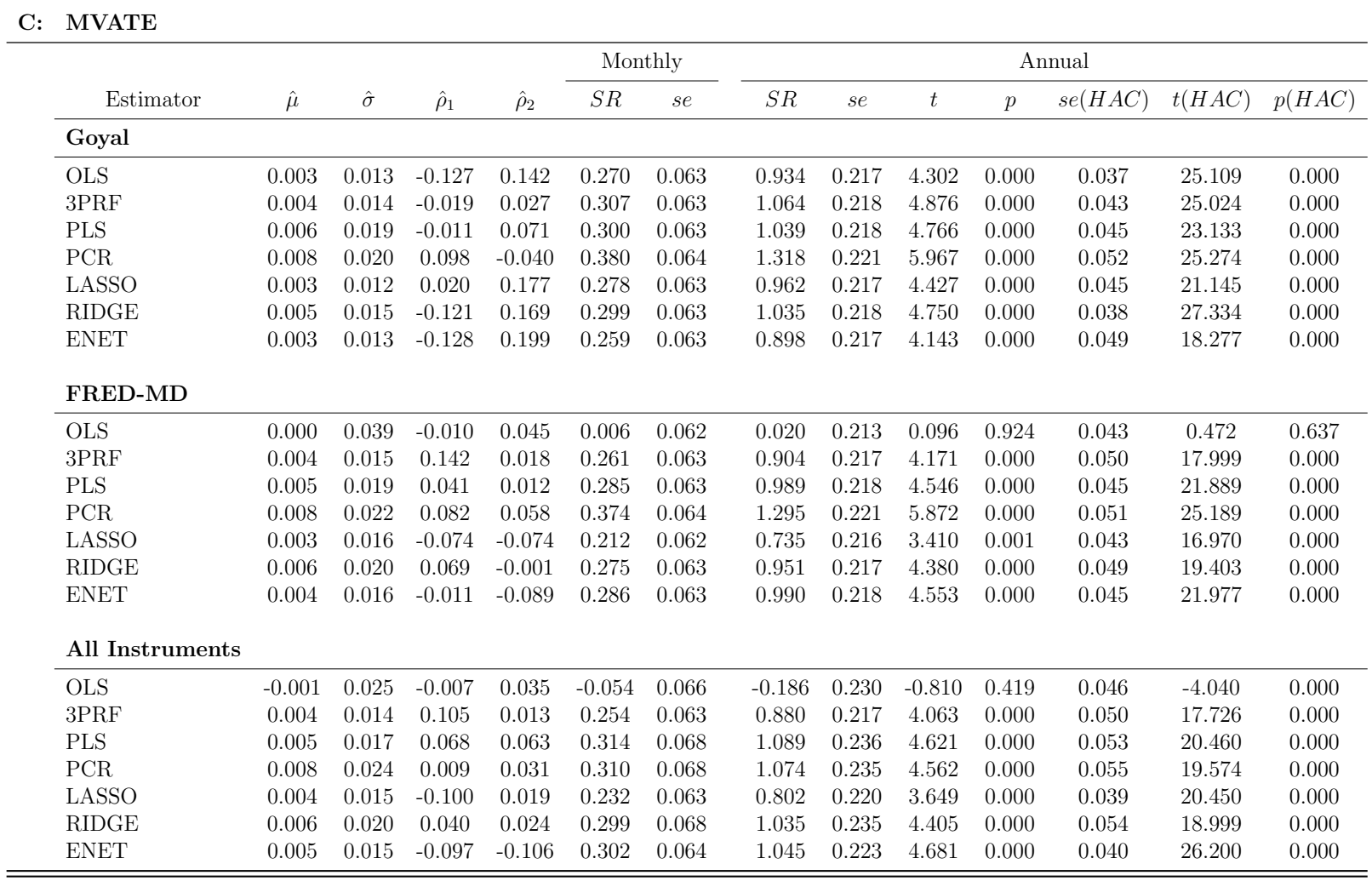

Table C.4 4 reports some summary statistics of the OOS (Jan-1996 to Dec-2017) for the 25 portfolios formed on size and book-to-market by estimator and three different set of conditioning information (Goyal, FRED-MD and "All Instruments"). Each panel shows the test of pairs of Sharpe ratios for three different framework to build efficient portfolios. Panel A reports conditionally mean-variance (CMV) efficient portfolios. Panel $\mathrm{B}$ reports unconditionally mean-variance efficient portfolios (UMV). Panel C presents the mean-variance tracking error (MVATE) portfolios. We split the results depending on conditioning information set used. Goyal variables comprises: $b / m$, $d f r$, dfy, infl, ltr, lty, ntis, svar, tms and $t b l$. The FRED-MD is a large dataset containing 128 macroeconomic and financial variables. Finally, "All Instruments" is the combination of Goyal and FRED-MD datasets with the Economic Policy Uncertainty (EPU) index and the Financial Stress Indicator (FSI). The table reports the monthly returns $(\hat{\mu})$, monthly returns $(\hat{\sigma})$, first and second autocorrelation $\left(\hat{\rho}_{1}\right.$ and $\hat{\rho}_{2}$ ) of the monthly returns. The Sharpe ratios $(S R)$ are presented monthly and annualized, as well as the standard error $(\mathrm{se})$. For the sake of completeness, it is also reported the t-statistic $(t)$, the standard p-value $(p)$, as well as the HAC standard error $(s e(H A C))$, the t-statistic obtained using HAC $(t(H A C))$ and its p-value $(p(H A C))$. 
Table C.5: Results for the 100 Portfolios Formed on Size and Book-to-Market

\begin{tabular}{|c|c|c|c|c|c|c|c|c|c|c|c|c|c|c|}
\hline \multirow[t]{3}{*}{ A: } & \multicolumn{14}{|l|}{ CMV } \\
\hline & \multirow[b]{2}{*}{ Estimator } & \multirow[b]{2}{*}{$\hat{\mu}$} & \multirow[b]{2}{*}{$\hat{\sigma}$} & \multirow[b]{2}{*}{$\hat{\rho}_{1}$} & \multirow[b]{2}{*}{$\hat{\rho}_{2}$} & \multicolumn{2}{|c|}{ Monthly } & \multicolumn{7}{|c|}{ Annual } \\
\hline & & & & & & $S R$ & se & $S R$ & se & $t$ & $p$-val & $s e(H A C)$ & $t(H A C)$ & $p(H A C)$ \\
\hline & \multicolumn{14}{|l|}{ Goyal } \\
\hline & OLS & 0.001 & 0.009 & -0.175 & 0.106 & 0.076 & 0.062 & 0.262 & 0.214 & 1.227 & 0.221 & 0.072 & 3.635 & 0.000 \\
\hline & $3 \mathrm{PRF}$ & 0.001 & 0.012 & -0.208 & 0.065 & 0.117 & 0.062 & 0.404 & 0.214 & 1.890 & 0.060 & 0.095 & 4.269 & 0.000 \\
\hline & PLS & 0.002 & 0.010 & -0.002 & 0.009 & 0.245 & 0.062 & 0.848 & 0.216 & 3.920 & 0.000 & 0.151 & 5.624 & 0.000 \\
\hline & PCR & 0.003 & 0.009 & 0.104 & 0.090 & 0.303 & 0.063 & 1.048 & 0.218 & 4.808 & 0.000 & 0.127 & 8.270 & 0.000 \\
\hline & LASSO & 0.001 & 0.009 & -0.051 & -0.002 & 0.087 & 0.062 & 0.302 & 0.214 & 1.415 & 0.158 & 0.096 & 3.140 & 0.002 \\
\hline & RIDGE & 0.001 & 0.013 & -0.027 & 0.010 & 0.060 & 0.062 & 0.207 & 0.213 & 0.970 & 0.333 & 0.082 & 2.512 & 0.013 \\
\hline & ENET & 0.001 & 0.009 & 0.047 & 0.022 & 0.097 & 0.062 & 0.336 & 0.214 & 1.573 & 0.117 & 0.101 & 3.314 & 0.001 \\
\hline & \multicolumn{14}{|l|}{ FRED-MD } \\
\hline & OLS & 0.000 & 0.001 & 0.185 & 0.005 & 0.128 & 0.062 & 0.443 & 0.214 & 2.068 & 0.040 & 0.065 & 6.830 & 0.000 \\
\hline & $3 \mathrm{PRF}$ & 0.002 & 0.007 & -0.255 & 0.134 & 0.210 & 0.062 & 0.728 & 0.216 & 3.376 & 0.001 & 0.042 & 17.141 & 0.000 \\
\hline & PLS & 0.002 & 0.007 & 0.081 & 0.283 & 0.350 & 0.063 & 1.211 & 0.220 & 5.513 & 0.000 & 0.060 & 20.049 & 0.000 \\
\hline & PCR & 0.002 & 0.011 & 0.072 & 0.081 & 0.203 & 0.062 & 0.703 & 0.215 & 3.266 & 0.001 & 0.144 & 4.898 & 0.000 \\
\hline & LASSO & 0.001 & 0.003 & 0.049 & 0.115 & 0.312 & 0.063 & 1.079 & 0.218 & 4.943 & 0.000 & 0.041 & 26.408 & 0.000 \\
\hline & RIDGE & 0.001 & 0.005 & -0.027 & 0.082 & 0.274 & 0.063 & 0.950 & 0.217 & 4.375 & 0.000 & 0.062 & 15.315 & 0.000 \\
\hline & ENET & 0.001 & 0.004 & -0.027 & 0.139 & 0.307 & 0.063 & 1.064 & 0.218 & 4.878 & 0.000 & 0.037 & 28.613 & 0.000 \\
\hline & \multicolumn{14}{|c|}{ All Instruments } \\
\hline & OLS & 0.000 & 0.001 & 0.211 & -0.001 & 0.118 & 0.067 & 0.409 & 0.231 & 1.773 & 0.078 & 0.082 & 4.972 & 0.000 \\
\hline & $3 \mathrm{PRF}$ & 0.002 & 0.007 & -0.188 & 0.220 & 0.240 & 0.062 & 0.832 & 0.216 & 3.846 & 0.000 & 0.049 & 16.828 & 0.000 \\
\hline & PLS & 0.002 & 0.006 & 0.106 & 0.049 & 0.309 & 0.068 & 1.072 & 0.235 & 4.554 & 0.000 & 0.074 & 14.532 & 0.000 \\
\hline & PCR & 0.003 & 0.010 & 0.119 & 0.031 & 0.269 & 0.068 & 0.932 & 0.234 & 3.983 & 0.000 & 0.154 & 6.069 & 0.000 \\
\hline & LASSO & 0.001 & 0.004 & 0.179 & 0.223 & 0.323 & 0.068 & 1.120 & 0.236 & 4.747 & 0.000 & 0.042 & 26.651 & 0.000 \\
\hline & RIDGE & 0.001 & 0.005 & -0.042 & 0.147 & 0.243 & 0.067 & 0.842 & 0.233 & 3.608 & 0.000 & 0.038 & 21.963 & 0.000 \\
\hline & ENET & 0.001 & 0.004 & 0.212 & 0.261 & 0.359 & 0.068 & 1.243 & 0.237 & 5.241 & 0.000 & 0.040 & 31.397 & 0.000 \\
\hline \multicolumn{15}{|c|}{ UMV } \\
\hline & & & & & & \multicolumn{2}{|c|}{ Monthly } & \multicolumn{7}{|c|}{ Annual } \\
\hline & Estimator & $\hat{\mu}$ & $\hat{\sigma}$ & $\hat{\rho}_{1}$ & $\hat{\rho}_{2}$ & $S R$ & se & $S R$ & se & $t$ & $p$ & $s e(H A C)$ & $t(H A C)$ & $p(H A C)$ \\
\hline & Goyal & & & & & & & & & & & & & \\
\hline & OLS & 0.001 & 0.009 & -0.175 & 0.102 & 0.072 & 0.062 & 0.248 & 0.213 & 1.164 & 0.245 & 0.072 & 3.471 & 0.001 \\
\hline & $3 \mathrm{PRF}$ & 0.001 & 0.010 & -0.165 & 0.086 & 0.133 & 0.062 & 0.462 & 0.214 & 2.157 & 0.032 & 0.104 & 4.445 & 0.000 \\
\hline & PLS & 0.002 & 0.010 & 0.002 & 0.017 & 0.241 & 0.062 & 0.836 & 0.216 & 3.867 & 0.000 & 0.154 & 5.439 & 0.000 \\
\hline & PCR & 0.003 & 0.009 & 0.132 & 0.109 & 0.315 & 0.063 & 1.090 & 0.218 & 4.992 & 0.000 & 0.110 & 9.935 & 0.000 \\
\hline & LASSO & 0.001 & 0.008 & -0.026 & 0.014 & 0.100 & 0.062 & 0.346 & 0.214 & 1.617 & 0.107 & 0.104 & 3.327 & 0.001 \\
\hline & RIDGE & 0.001 & 0.011 & -0.010 & 0.036 & 0.083 & 0.062 & 0.289 & 0.214 & 1.351 & 0.178 & 0.095 & 3.031 & 0.003 \\
\hline & ENET & 0.001 & 0.008 & 0.058 & 0.040 & 0.103 & 0.062 & 0.357 & 0.214 & 1.669 & 0.096 & 0.104 & 3.444 & 0.001 \\
\hline & FRED-MD & & & & & & & & & & & & & \\
\hline & OLS & 0.000 & 0.001 & 0.186 & 0.006 & 0.128 & 0.062 & 0.442 & 0.214 & 2.064 & 0.040 & 0.065 & 6.802 & 0.000 \\
\hline & $3 \mathrm{PRF}$ & 0.001 & 0.008 & -0.266 & 0.128 & 0.197 & 0.062 & 0.684 & 0.215 & 3.177 & 0.002 & 0.039 & 17.320 & 0.000 \\
\hline & PLS & 0.002 & 0.007 & 0.065 & 0.297 & 0.346 & 0.063 & 1.199 & 0.220 & 5.463 & 0.000 & 0.056 & 21.277 & 0.000 \\
\hline & PCR & 0.002 & 0.010 & 0.070 & 0.082 & 0.209 & 0.062 & 0.723 & 0.216 & 3.356 & 0.001 & 0.140 & 5.185 & 0.000 \\
\hline & LASSO & 0.001 & 0.003 & 0.055 & 0.123 & 0.305 & 0.063 & 1.057 & 0.218 & 4.846 & 0.000 & 0.040 & 26.500 & 0.000 \\
\hline & RIDGE & 0.001 & 0.005 & -0.033 & 0.087 & 0.265 & 0.063 & 0.918 & 0.217 & 4.232 & 0.000 & 0.059 & 15.576 & 0.000 \\
\hline & ENET & 0.001 & 0.004 & -0.037 & 0.141 & 0.301 & 0.063 & 1.044 & 0.218 & 4.789 & 0.000 & 0.036 & 28.759 & 0.000 \\
\hline & All Instrume & & & & & & & & & & & & & \\
\hline & OLS & 0.000 & 0.001 & 0.211 & -0.001 & 0.118 & 0.067 & 0.409 & 0.231 & 1.771 & 0.078 & 0.082 & 4.959 & 0.000 \\
\hline & $3 \mathrm{PRF}$ & 0.002 & 0.007 & -0.194 & 0.210 & 0.228 & 0.062 & 0.791 & 0.216 & 3.663 & 0.000 & 0.047 & 16.654 & 0.000 \\
\hline & PLS & 0.002 & 0.005 & 0.134 & 0.062 & 0.315 & 0.068 & 1.092 & 0.236 & 4.634 & 0.000 & 0.066 & 16.579 & 0.000 \\
\hline & PCR & 0.002 & 0.009 & 0.131 & 0.007 & 0.272 & 0.068 & 0.944 & 0.234 & 4.031 & 0.000 & 0.136 & 6.925 & 0.000 \\
\hline & LASSO & 0.001 & 0.004 & 0.184 & 0.234 & 0.315 & 0.068 & 1.090 & 0.236 & 4.626 & 0.000 & 0.042 & 25.906 & 0.000 \\
\hline & RIDGE & 0.001 & 0.005 & -0.021 & 0.165 & 0.247 & 0.067 & 0.857 & 0.233 & 3.670 & 0.000 & 0.037 & 23.256 & 0.000 \\
\hline & ENET & 0.001 & 0.004 & 0.222 & 0.280 & 0.352 & 0.068 & 1.218 & 0.237 & 5.142 & 0.000 & 0.040 & 30.241 & 0.000 \\
\hline
\end{tabular}

(Continued) 
Table C.5: Results for the 100 Portfolios Formed on Size and Book-to-Market (Continued)

\begin{tabular}{|c|c|c|c|c|c|c|c|c|c|c|c|c|c|}
\hline \multirow[b]{2}{*}{ Estimator } & \multirow[b]{2}{*}{$\hat{\mu}$} & \multirow[b]{2}{*}{$\hat{\sigma}$} & \multirow[b]{2}{*}{$\hat{\rho}_{1}$} & \multirow[b]{2}{*}{$\hat{\rho}_{2}$} & \multicolumn{2}{|c|}{ Monthly } & \multicolumn{7}{|c|}{ Annual } \\
\hline & & & & & $S R$ & se & $S R$ & se & $t$ & $p$ & $s e(H A C)$ & $t(H A C)$ & $p(H A C)$ \\
\hline \multicolumn{14}{|l|}{ Goyal } \\
\hline OLS & 0.000 & 0.024 & -0.094 & 0.078 & 0.003 & 0.062 & 0.010 & 0.213 & 0.047 & 0.962 & 0.042 & 0.239 & 0.811 \\
\hline $3 \mathrm{PRF}$ & 0.002 & 0.026 & -0.175 & 0.064 & 0.059 & 0.062 & 0.204 & 0.213 & 0.955 & 0.341 & 0.060 & 3.374 & 0.001 \\
\hline PLS & 0.005 & 0.017 & 0.042 & 0.035 & 0.279 & 0.063 & 0.965 & 0.217 & 4.440 & 0.000 & 0.101 & 9.541 & 0.000 \\
\hline PCR & 0.005 & 0.017 & 0.103 & 0.108 & 0.312 & 0.063 & 1.082 & 0.218 & 4.957 & 0.000 & 0.098 & 11.065 & 0.000 \\
\hline LASSO & 0.001 & 0.017 & -0.016 & 0.048 & 0.084 & 0.062 & 0.292 & 0.214 & 1.365 & 0.173 & 0.089 & 3.263 & 0.001 \\
\hline RIDGE & 0.001 & 0.026 & -0.010 & 0.060 & 0.044 & 0.062 & 0.154 & 0.213 & 0.720 & 0.472 & 0.070 & 2.197 & 0.029 \\
\hline ENET & 0.001 & 0.021 & 0.014 & 0.026 & 0.043 & 0.062 & 0.150 & 0.213 & 0.703 & 0.483 & 0.067 & 2.231 & 0.027 \\
\hline \multicolumn{14}{|l|}{ FRED-MD } \\
\hline OLS & 0.003 & 0.069 & -0.136 & 0.022 & 0.047 & 0.062 & 0.163 & 0.213 & 0.763 & 0.446 & 0.025 & 6.613 & 0.000 \\
\hline $3 \mathrm{PRF}$ & 0.003 & 0.023 & -0.220 & 0.009 & 0.135 & 0.062 & 0.469 & 0.214 & 2.190 & 0.029 & 0.015 & 31.078 & 0.000 \\
\hline PLS & 0.004 & 0.019 & 0.094 & 0.210 & 0.230 & 0.062 & 0.796 & 0.216 & 3.683 & 0.000 & 0.101 & 7.858 & 0.000 \\
\hline PCR & 0.004 & 0.022 & 0.082 & 0.084 & 0.198 & 0.062 & 0.687 & 0.215 & 3.189 & 0.002 & 0.139 & 4.931 & 0.000 \\
\hline LASSO & 0.002 & 0.008 & 0.074 & 0.113 & 0.240 & 0.062 & 0.833 & 0.216 & 3.851 & 0.000 & 0.037 & 22.748 & 0.000 \\
\hline RIDGE & 0.003 & 0.015 & -0.034 & 0.082 & 0.188 & 0.062 & 0.652 & 0.215 & 3.030 & 0.003 & 0.029 & 22.333 & 0.000 \\
\hline ENET & 0.001 & 0.012 & 0.192 & 0.065 & 0.129 & 0.062 & 0.448 & 0.214 & 2.092 & 0.037 & 0.069 & 6.465 & 0.000 \\
\hline \multicolumn{14}{|c|}{ All Instruments } \\
\hline OLS & -0.002 & 0.085 & -0.103 & -0.020 & -0.026 & 0.066 & -0.091 & 0.230 & -0.394 & 0.694 & 0.040 & -2.284 & 0.023 \\
\hline $3 \mathrm{PRF}$ & 0.003 & 0.020 & -0.187 & 0.084 & 0.145 & 0.062 & 0.502 & 0.214 & 2.344 & 0.020 & 0.026 & 19.497 & 0.000 \\
\hline PLS & 0.003 & 0.013 & 0.110 & -0.064 & 0.263 & 0.068 & 0.912 & 0.234 & 3.898 & 0.000 & 0.046 & 19.874 & 0.000 \\
\hline PCR & 0.005 & 0.020 & 0.125 & 0.031 & 0.271 & 0.068 & 0.937 & 0.234 & 4.003 & 0.000 & 0.140 & 6.698 & 0.000 \\
\hline LASSO & 0.003 & 0.010 & 0.117 & 0.200 & 0.253 & 0.067 & 0.875 & 0.234 & 3.748 & 0.000 & 0.036 & 24.412 & 0.000 \\
\hline RIDGE & 0.002 & 0.015 & -0.049 & 0.083 & 0.117 & 0.067 & 0.407 & 0.231 & 1.762 & 0.079 & 0.036 & 11.442 & 0.000 \\
\hline ENET & 0.002 & 0.012 & -0.021 & -0.093 & 0.179 & 0.067 & 0.620 & 0.232 & 2.675 & 0.008 & 0.060 & 10.313 & 0.000 \\
\hline
\end{tabular}

Table C.5 reports some summary statistics of the OOS (Jan-1996 to Dec-2017) for the 100 portfolios formed on size and book-to-market by estimator and three different set of conditioning information (Goyal, FREDMD and "All Instruments"). Each panel shows the test of pairs of Sharpe ratios for three different framework to build efficient portfolios. Panel A reports conditionally mean-variance (CMV) efficient portfolios. Panel $\mathrm{B}$ reports unconditionally mean-variance efficient portfolios (UMV). Panel C presents the mean-variance tracking error (MVATE) portfolios. We split the results depending on conditioning information set used. Goyal variables comprises: $b / m$, $d f r$, dfy, infl, ltr, lty, ntis, svar, tms and $t b l$. The FRED-MD is a large dataset containing 128 macroeconomic and financial variables. Finally, "All Instruments" is the combination of Goyal and FRED-MD datasets with the Economic Policy Uncertainty (EPU) index and the Financial Stress Indicator (FSI). The table reports the monthly returns $(\hat{\mu})$, monthly returns $(\hat{\sigma})$, first and second autocorrelation $\left(\hat{\rho}_{1}\right.$ and $\hat{\rho}_{2}$ ) of the monthly returns. The Sharpe ratios $(S R)$ are presented monthly and annualized, as well as the standard error $(\mathrm{se})$. For the sake of completeness, it is also reported the t-statistic $(t)$, the standard p-value $(p)$, as well as the HAC standard error $(s e(H A C))$, the t-statistic obtained using $\mathrm{HAC}(t(H A C))$ and its p-value $(p(H A C))$. 